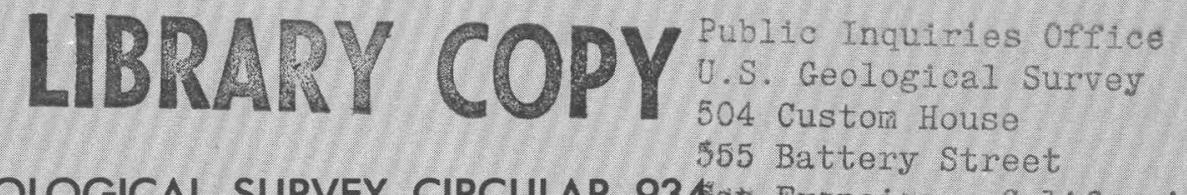

U.S. GEOLOGICAL SURVEY CIRCULAR 934ati Francisco, California

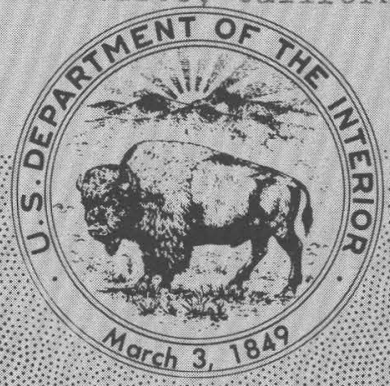

Worldwide Directory of National Earth-Science Agencies and Related International Organizations 



\section{Worldwide Directory of National Earth-Science Agencies and Related International Organizations}

Compiled by E. J. Tinsley and Joyce P. Hollander

A listing of governmental earth-science agencies and selected major international organizations whose functions are similar to those of the U.S. Geological Survey

U.S. GEOLOGICAL SURVEY CIRCULAR 934 


\section{Department of the Interior}

\section{WILLIAM P. CLARK, Secretary}

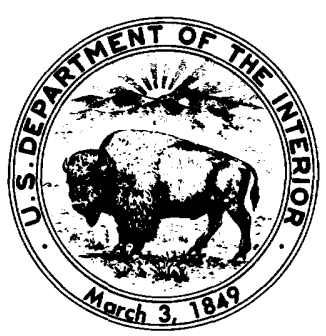

\section{U.S. Geological Survey}

Dallas L. Peck, Director

\section{Library of Congress Cataloging in Publication Data}

Tinsley, Elizabeth J.

Worldwide directory of national earth-science agencies and related international organizations.

(U.S. Geological Survey circular ; 934)

Supt. of Docs. no.: I 19.4/2:934

1. Earth sciences-Directories. 2. Geological surveys-Directories. I. Hollander, Joyce P. II. Title. III. Series. 84-600161

Free on application to Distribution Branch, Text Products Section, U. S. Geological Survey, 604 South Pickett Street, Alexandria, VA 22304 


\section{CONTENTS}

Page

Introduction

Afghanistan

Albania

Algeria

Angola

Argentina

Australia

Austria

Bahamas, The

Bangladesh

Barbados

Belgium

Belize

Benin

Bhutan

Bolivia -

Botswana

Brazil

Brunei

Bulgaria

Burma

Burundi

Cameroon --

Canada

Central African Republic

Chad

Chile

China --

Colombia

Congo

Cook Islands

Costa Rica

Cuba

Cyprus

Czechoslovakia

Denmark

Djibouti

Dominica

Dominican Republic

Ecuador

Egypt

El Salvador

Ethiopia

Fiji

Finland

France

French Guiana --_-_-_-

Gabon -

Gambia, The

German Democratic Republic

Germany, Federal Republic of

1 Ghana

13 Greece -

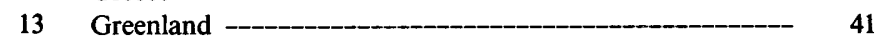

13 Guadeloupe -_____ 41

14 Guatemala -

15 Guinea -______ 42

16 Guinea-Bissau -_-_ 42

17 Guyana --_ 43

17 Haiti -_____- 43

18 Honduras --_-_- 44

18 Hong Kong -

19 Hungary -- 44

19 Iceland -

20 India -_- 46

20 Indonesia -

20 Iran -_-_-_-_-_ 48

21 Iraq -

22 Ireland -

23 Israel --_-_-_-_-_-_ 49

23 Italy -___ 50

24 Ivory Coast -_-_ 50

24 Jamaica -

25 Japan -

25 Jordan -_-__-_ 52

26 Kenya -_-_-_-_-_-_ 53

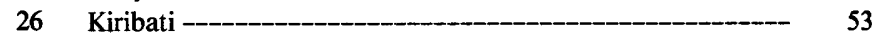

26 Korea, North _-_.______-_ 53

27 Korea, South _____________ 54

29 Kuwait _-_ 54

30 Laos -_-___-_ 55

30 Lebanon -

30 Lesotho --_-_-_-_-_-_-_-_ 55

31 Liberia _-________ 56

32 Libya -_-__-_ 56

32 Liechtenstein -______- 57

33 Luxembourg _-______- 57

33 Madagascar -_-_-_-_-_-_ 58

34 Malawi -_-_-____- 58

34 Malaysia -_____________- 59

34 Mali --_-_-_-_-_-_ 59

35 Malta -___- 60

35 Martinique - 60

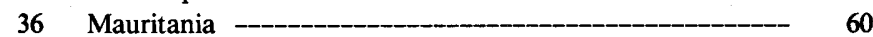

36 Mauritius -

37 Mexico -

37 Mongolia -

38 Morocco -_______- 63

38 Mozambique -

38 Namibia -_ 64

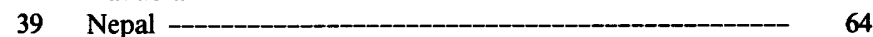

39 Netherlands, The - 64 
Netherlands Antilles

New Caledonia

New Zealand

Nicaragua

Niger

Nigeria

Norway

Oman

Pakistan

Panama

Papua New Guinea

Paraguay

Peru

Philippines, The

Poland

Portugal

Qatar

Reunion

Romania

Rwanda

Saudi Arabia

Senegal

Sierra Leone

Singapore

Solomon Islands

Somalia

South Africa

Soviet Union

Spain

Sri Lanka
Sudan

Suriname

Swaziland

Sweden

Switzerland

Syria

Tanzania

Thailand

Togo

Tonga, Kingdom of

Trinidad and Tobago

Tunisia

Turkey

Uganda

United Kingdom

United States of America

Upper Volta

Uruguay

Vanuatu

Venezuela

Vietnam

Western Samoa

Yemen, Arab Republic

Yemen, People's Democratic Republic

Yugoslavia

Zaire -

Zambia

Zimbabwe

Taiwan

Major international organizations
82

83

83

83

84

84

85

85

88

88

88

89

89

90

90

91

91

92

92

92

93

94

94

94

94

95

95

96

96

98

\section{ILLUSTRATIONS}

FiguRES I-X. Reference maps showing:

I. The world

II. North America

III. Central America and the Caribbean

IV. South America

V. Europe

VI. Middle East

VII. Africa

VIII. Soviet Union and East and South Asia

IX. Southeast Asia

X. Oceania 


\title{
Worldwide Directory of National Earth-Science Agencies and Related International Organizations
}

\author{
Compiled by E. J. Tinsley and Joyce P. Hollander
}

\section{INTRODUCTION}

The "Worldwide Directory" provides addresses of governmental earth-science agencies around the world that have functions similar to those of one or more of the operating divisions of the U.S. Geological Survey (USGS); it also lists addresses of major international organizations that are concerned with some phase of the earth sciences.

Most of the clata presented herein are current as of November 1983; inevitably, some of the names and addresses given have changed since our compilation. Much of the information was supplied by staff members of American embassies around the world, and a gratifying amount of data was provided by members of counterpart national organizations. We are indebted to all those officials, to all USGS colleagues, and to others who have kindly assisted us by providing information. Those countries for which we were unable to obtain recent accurate information from sources available to us are identified.
The directory is arranged alphabetically by country name. The location of each country is shown on a small index map, which is keyed to one of 10 reference maps showing the major regions of the world. The index maps should help the user to identify countries that have new names or that have recently attained independence.

Wherever it is feasible, names of agencies in a country whose language is based on the Latin alphabet are given in the language of that country. English translations have been added in brackets where they seem necessary. Names of agencies in a country whose language is based on an alphabet other than Latin are given in English.

The principal functions of the agencies listed are keyed to those of the USGS and are indicated by code letters in the left margin of the column, as follows:

$\mathrm{C}=$ Cartography

$\mathrm{G}=$ Geology

$\mathrm{H}=$ Hydrology

$\mathbf{R}=$ Minerals and petroleum regulation 


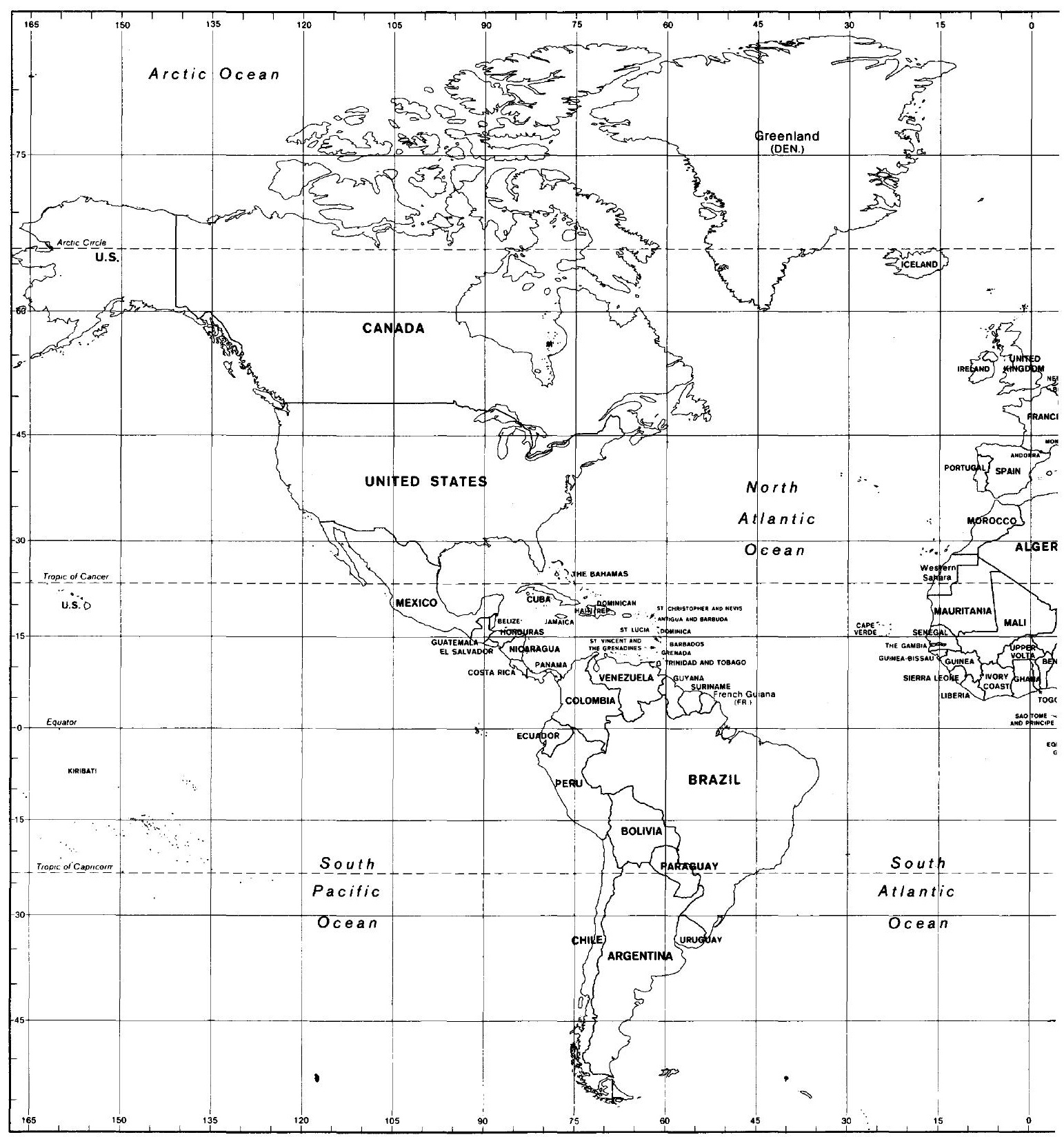


The World

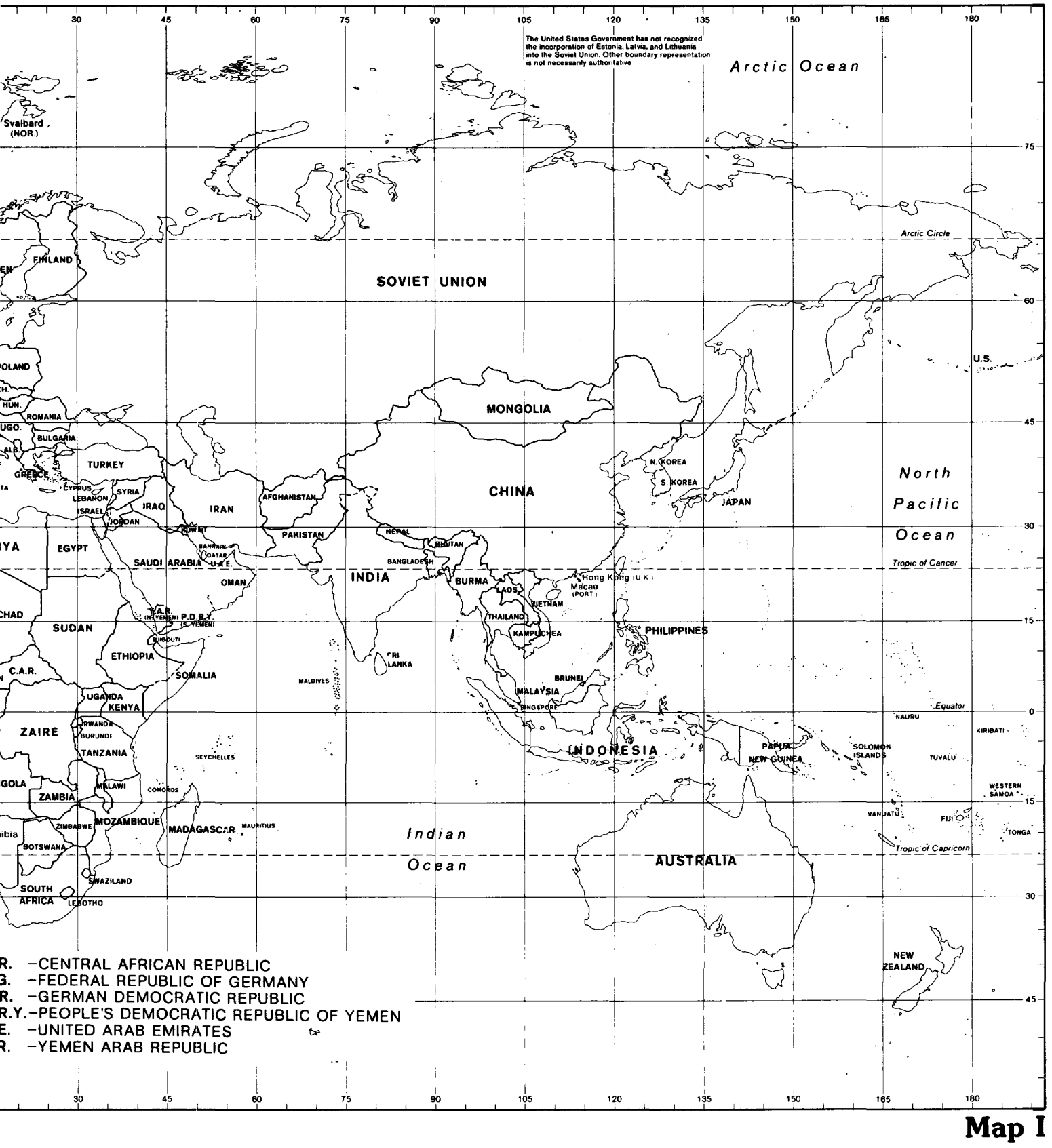




\section{North America}

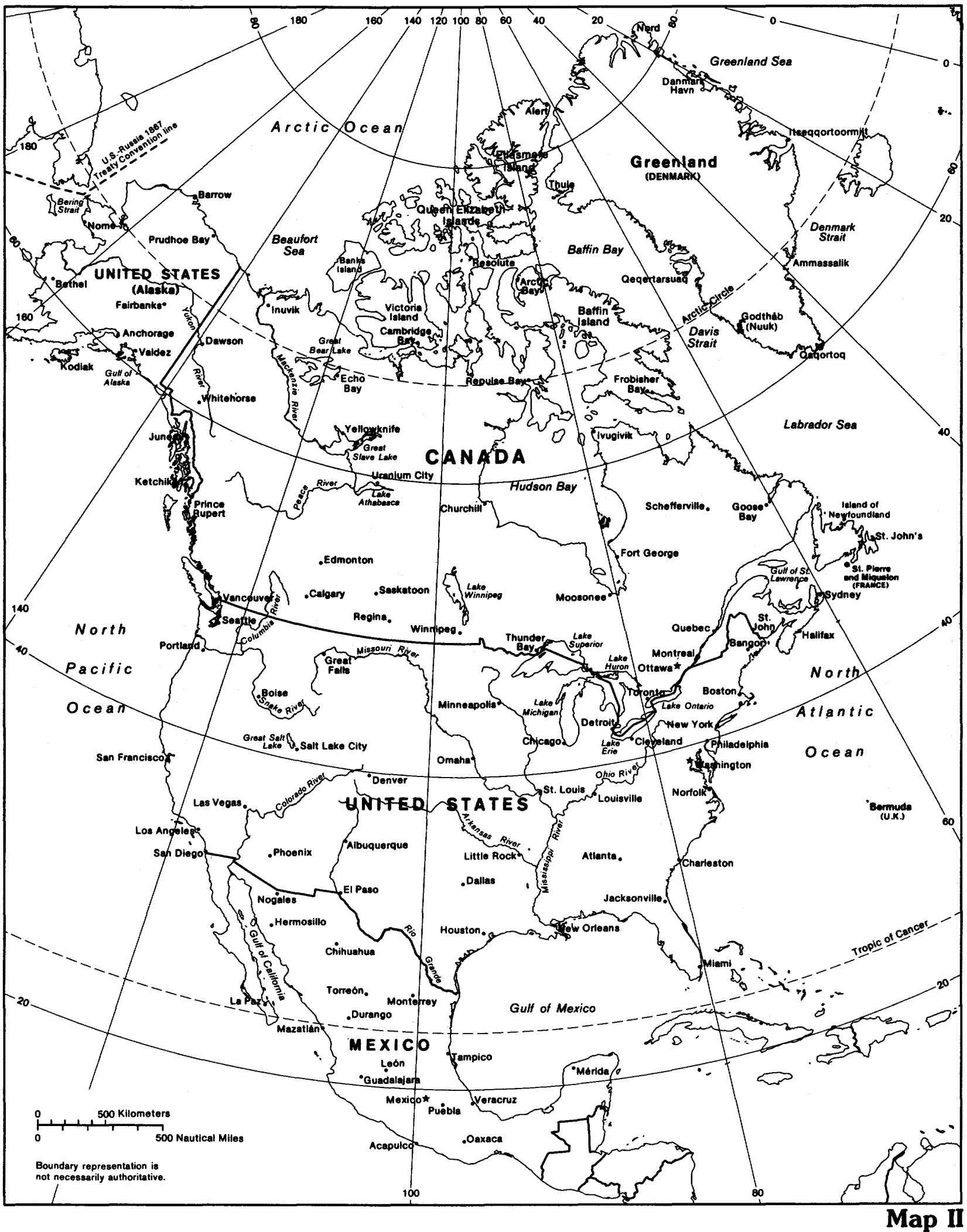




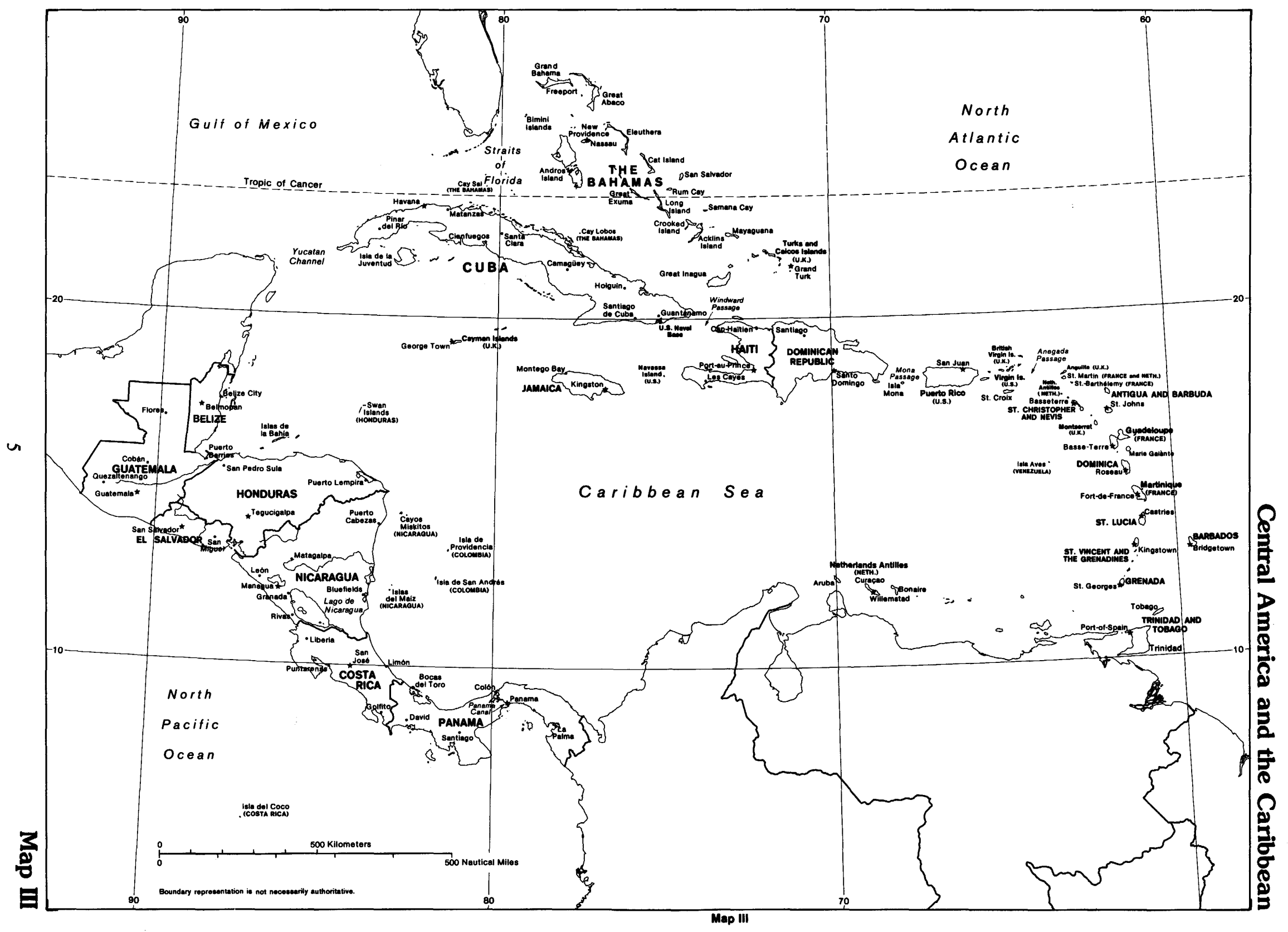




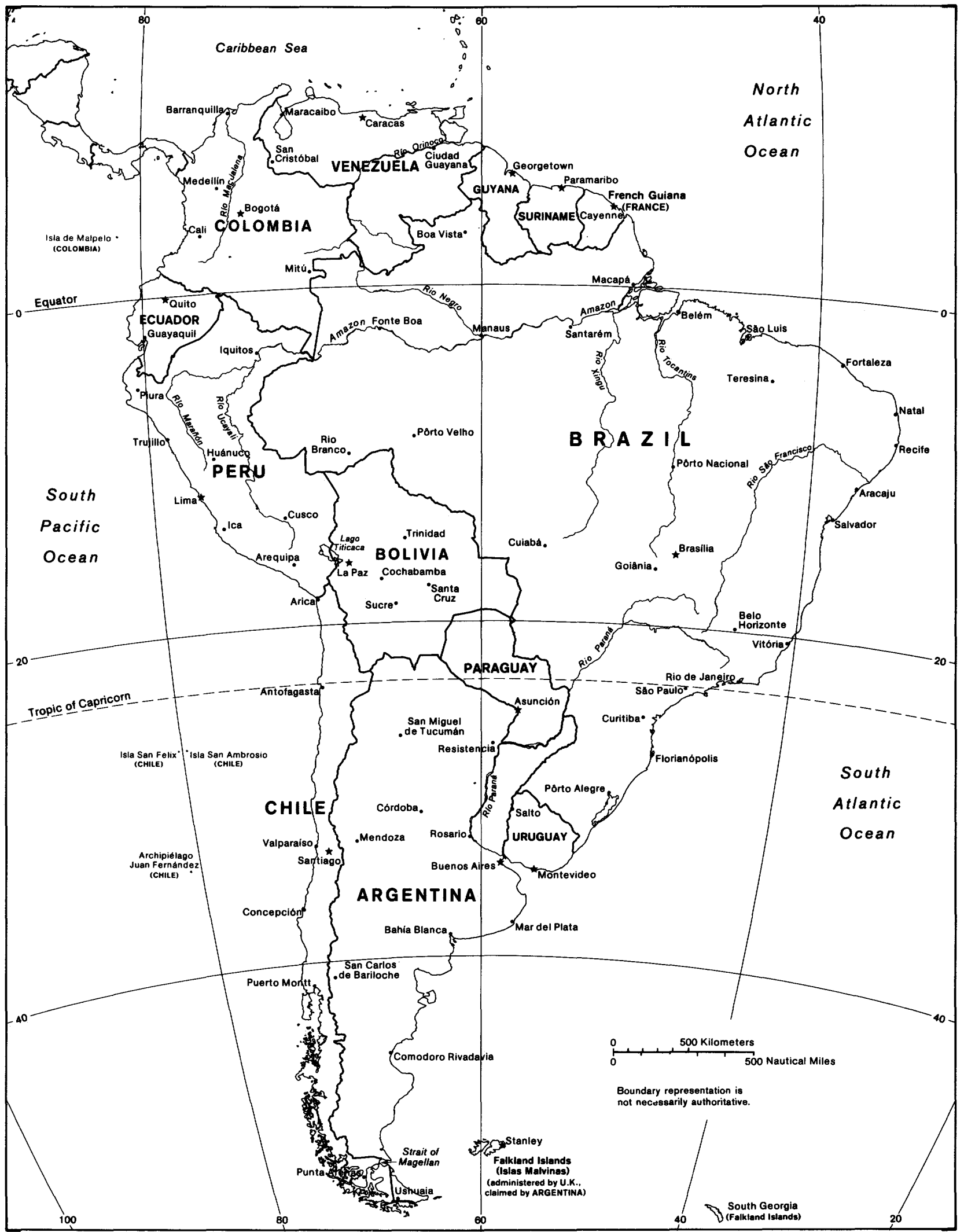


Europe

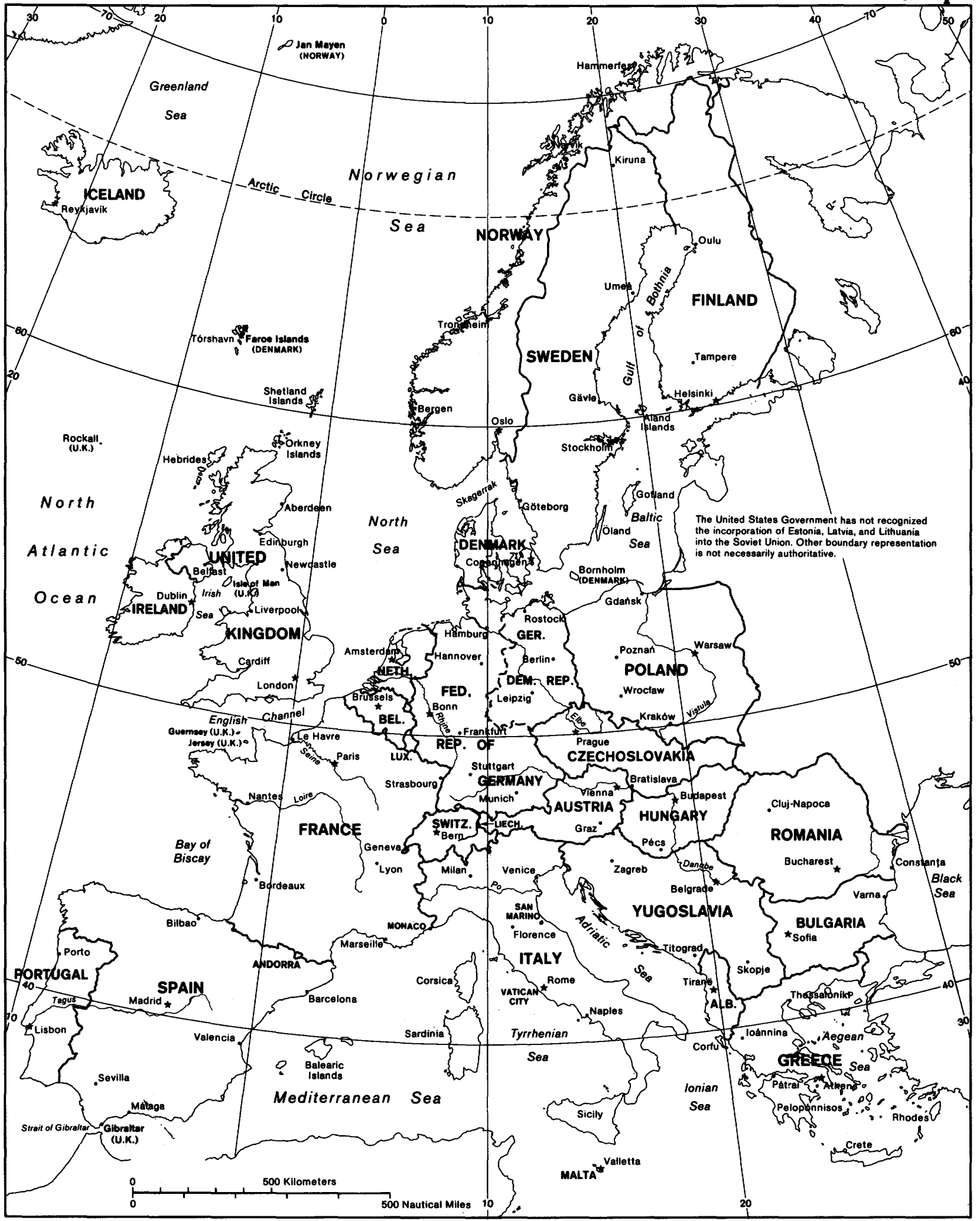

Map V 


\section{Middle East}

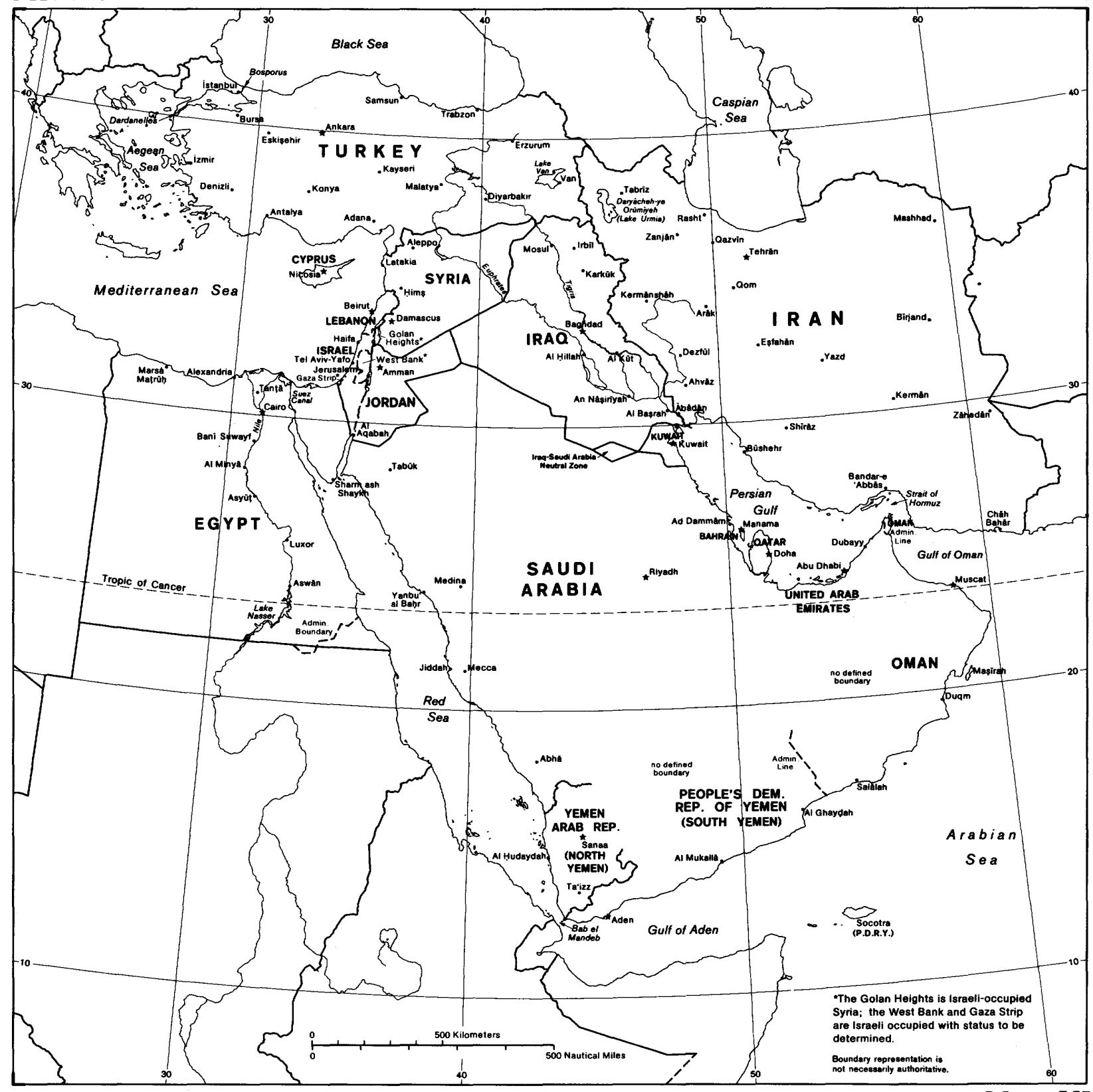




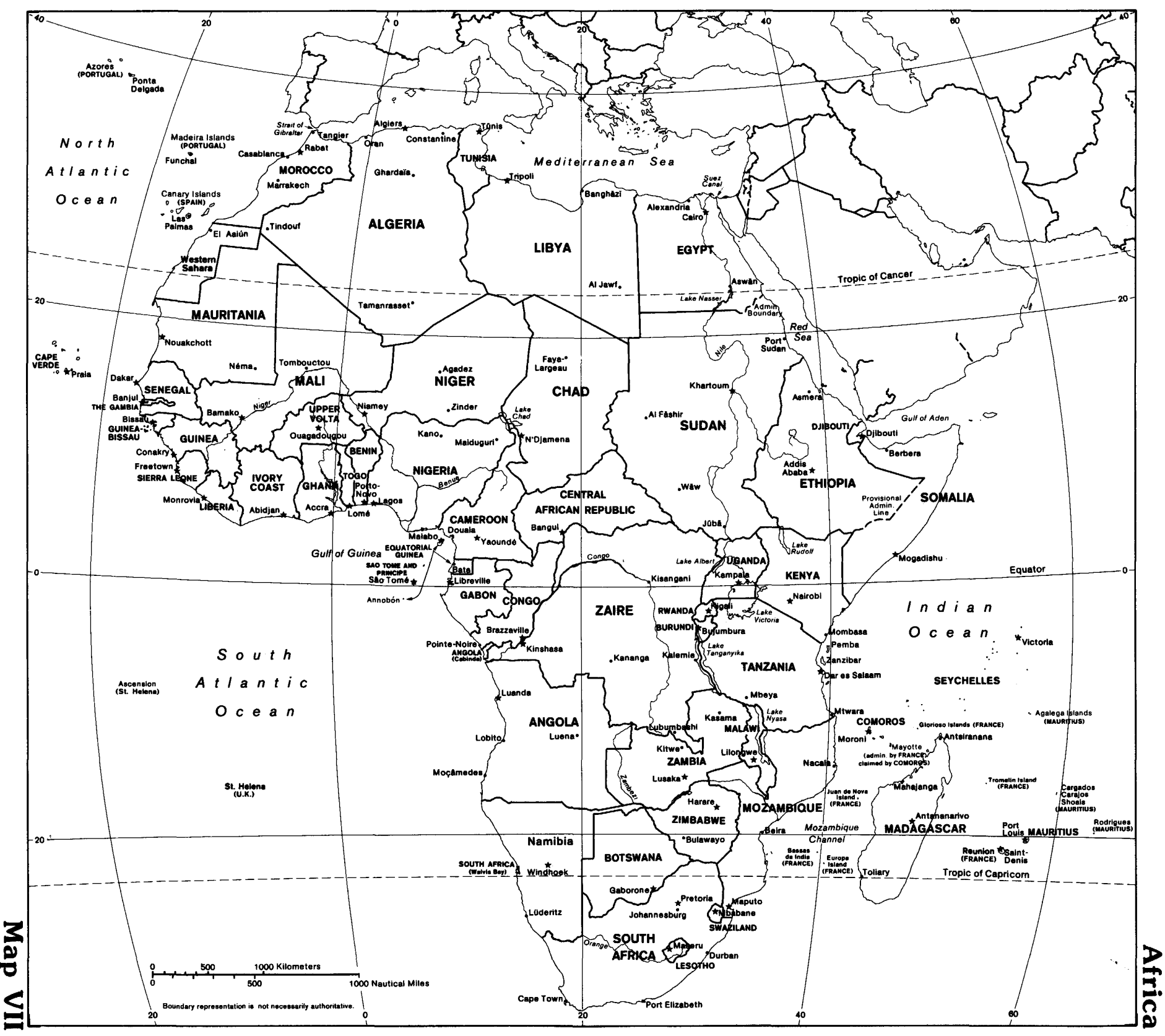


Soviet Union, East and South Asia

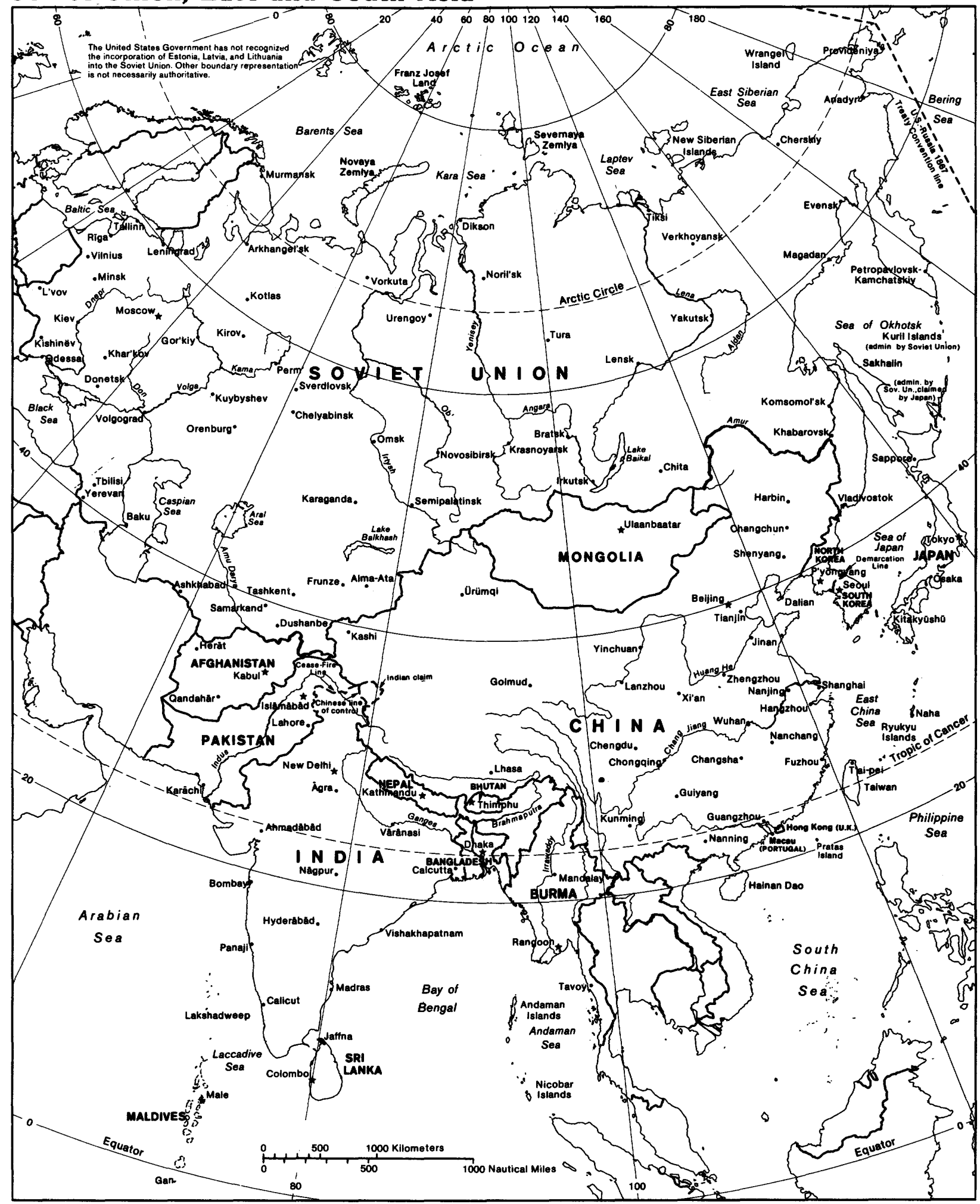

Map VIII 


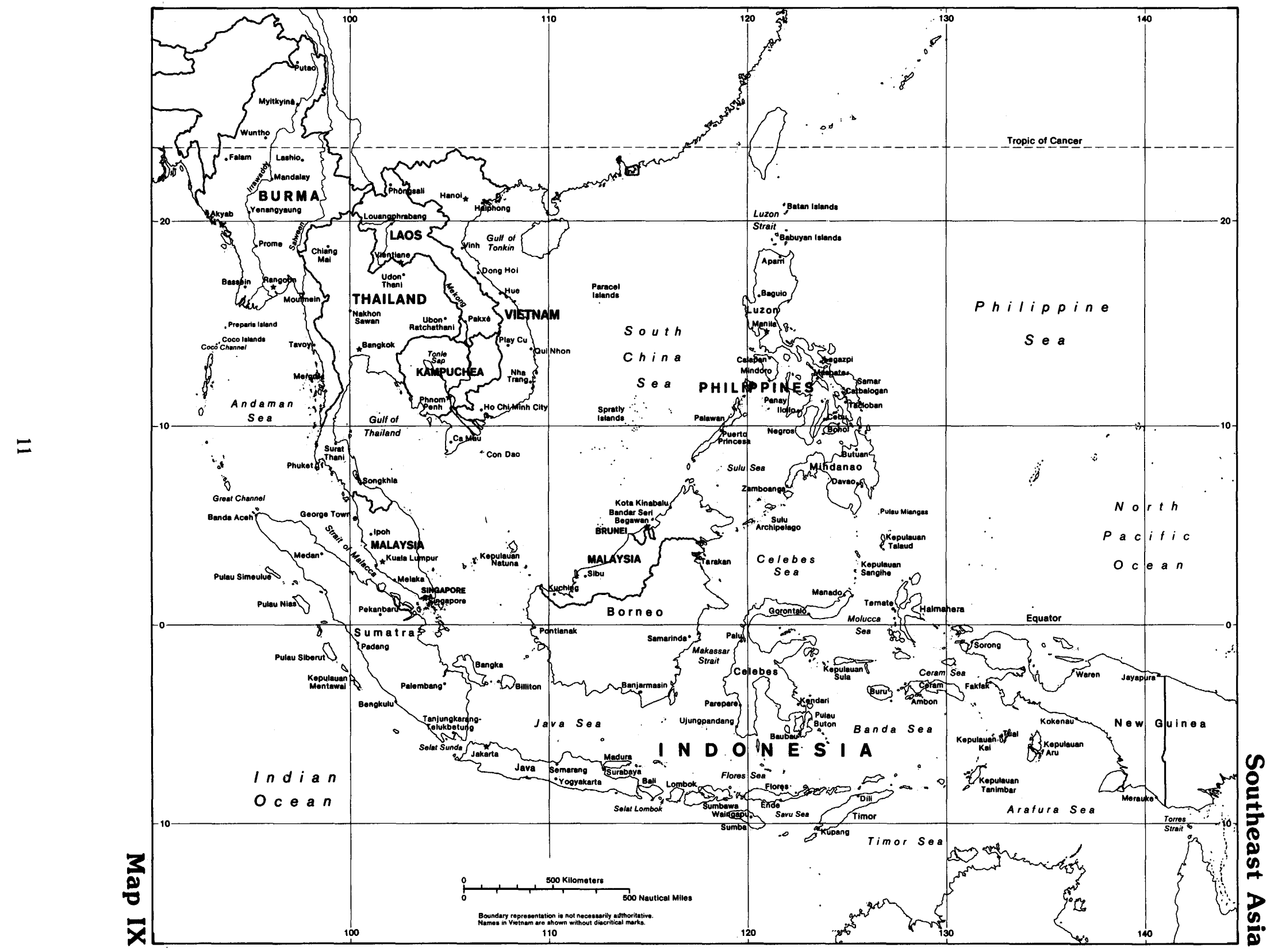




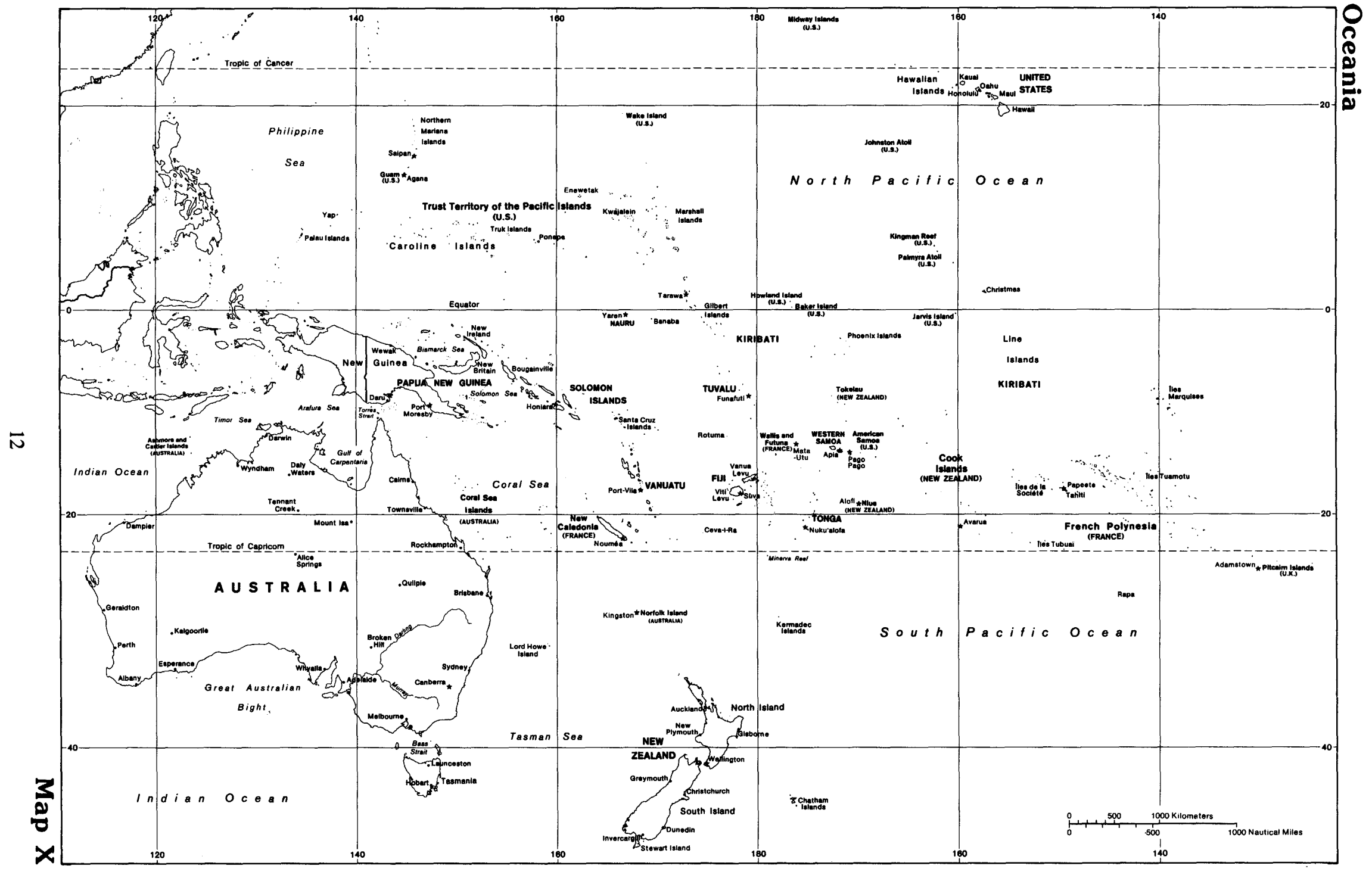




\section{AFGHANISTAN}

(No recent information)

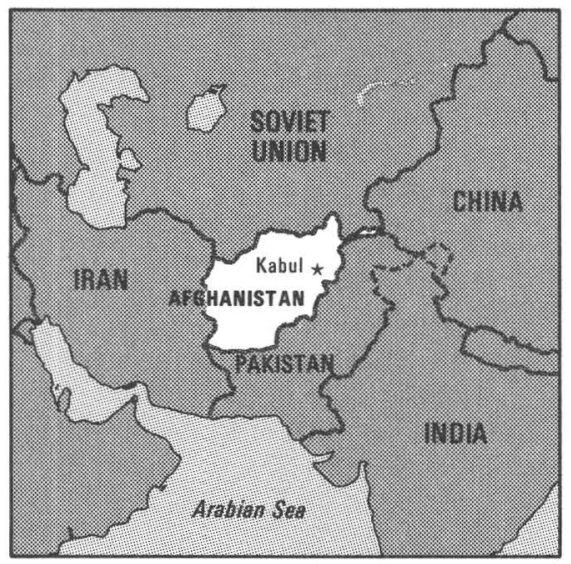

(See reference map VIII)

G

Department of Mines and Geology

Ministry of Mines and Industries

Darulaman

Kabul

President: Noor Ahmad Azimi

Ministry of Mines and Industries

Darulaman

Kabul

Minister: Abdullah Samah Salah

Ministry of Water and Power

Kabul

Minister: Jumma Mohammed Mohammadi

C

Cartographic and Cadastral Survey Institute Institute of Cartography

Kabul

Director: Amir Ahmad

R

\section{ALBANIA}

(No recent information)

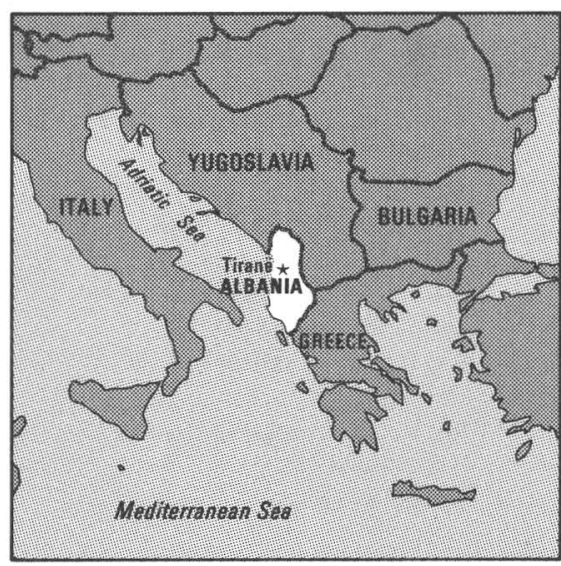

(See reference map $V$
G

C

$\mathrm{H}$

\section{ALGERIA}

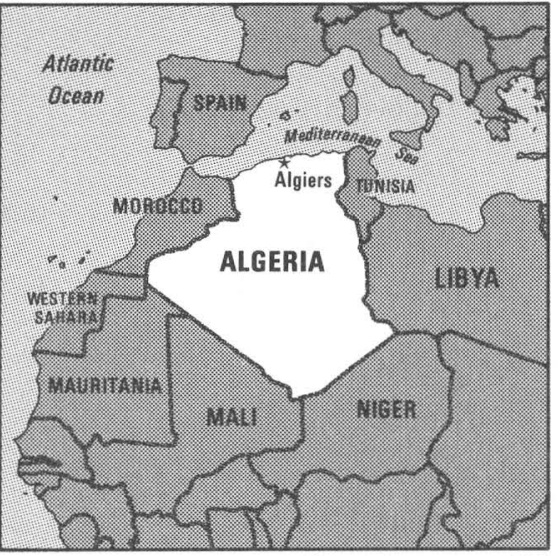

(See reference map VII)

G
Ministry of Industry and Mining

Tirane

Association of Scientific Workers of the

Tirane

President: Kol Paparisto

Instituti Hidrometéorologjik

214 rue Kongreasi Permetit

Tirane Peoples Republic of Albania

Afghan National Petroleum Company

Ministry of Mines and Industries

Kabul

President: Abul Samad Salah

Acting Deputy Minister Director-General: Hafizullah Nawabi 
Institut des Géo-Sciences

Universite de Sciences et de la Technologie d'Oran

B.P. 1505

El-M'Nouar - Oran

G

Centre National de Recherche et d'Applications des Géosciences

Universite d'Alger

2, rue Didouche Mourad

Alger

Directeur: Nacer-Eddine Kazi-Tani

G, H, Institut des Sciences de la Terre

Universite de Constantine

Ain El Bey - Constantine

G,H Institut de Biologie et des Sciences de la Terre

Universite d'Oran

Es-Senia - Oran

G,C Institut des Sciences de la Terre

Universite des Sciences et de la Technologie Houari Boudediene

B.P. 9

Dar Al Beida - Alger

Directeur: Mohamed Tefiani

H

$\mathrm{H}$

Centre National des Zones Arides

Beni Abbes - Bechar

Directeur: Mme N. Bounaga

Secrétariat d'Etat aux Forêts et a la Mise en Valeur des Terres

Immeuble des Forêts

Ex. Bois de Boulogne

El Madania, Alger

Secrétaire d'Etat: Mohamed Rouighi

$\mathrm{H} \quad$ Ministère de l'Hydraulique

Ex-Grand Seminaire

Kouba, Algiera

Minister: Brahim Brahimi

$\mathrm{H}$

Institut d'Hydraulique

Universite des Sciences et de ia Technologie d'Oran

B.P. 1505

El-M'Nouar - Oran

C

Ecole Nationale de Sciences eodesiques

B.P. 13

Arzew

Directeur: Rahel Redjouane

$\mathrm{H}$

Institut d'Hydraulique

Université des Sciences et de la Technologie Houari Boumediene

B.P. 9

Dar El Beida - Alger

$\mathrm{H}$
C

Institut National de Cartographie

123 rue de Tripoli

B.P. 32 Hussein-Dey

Algiers

Director: Halim Mansour

R

Ministère de l'Energie et des Industries Petrochimiques

80, Avenue Ahmed Ghermoul

Algiers

Minister: Belkacem Nabi

R

Ministère de l'Industrie Lourde

Immeuble "Le Colisée"

Rue Ahmed Bey de Constantine

Algiers

Minister: Merbah Kasdi

\section{ANGOLA}

(No recent information)

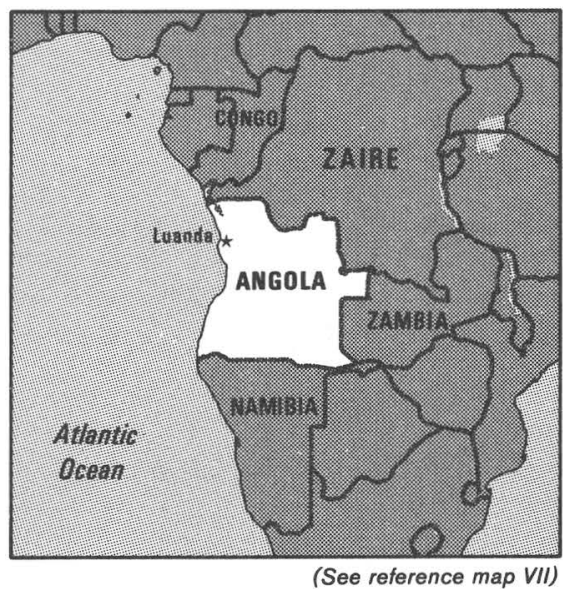

G,C,R Direcção de Serviços de Geología e Minas Caixa Postal 1260-C

Luanda

Director: Jorge Manuel Trigo de Mira

G

Observatório Meteorógico e Magnético

João Capelo

Rua Diogo Cão 20

Caixa Postal 1288-C

Director: Alberto Leão Diniz 


\section{ARGENTINA}

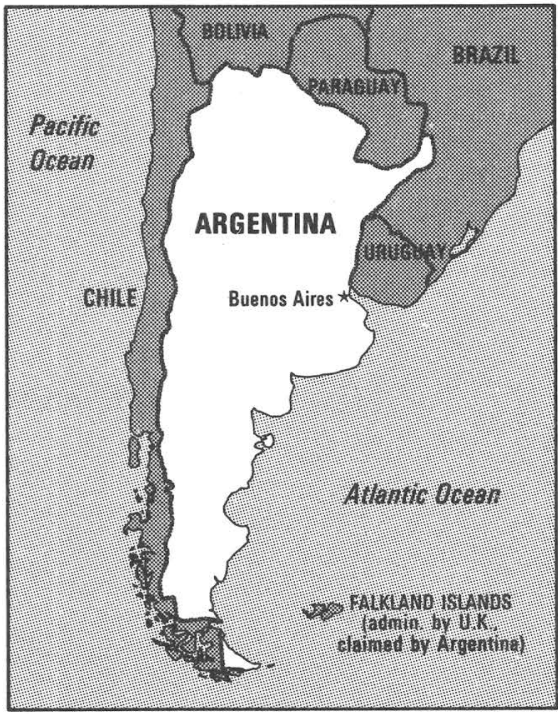

(See reference map IV)

Avenida Santa Fe $1548,4^{\circ}$ Piso

(1060) Buenos Aires

Director: Dr. Oscar Valentin Reverberi

Servicio Geológico Nacional

Secretaria de Estado de Mineria

Avenida Santa Fe 1548

1060 Buenos Aires

Director: Dr. Oscar J. Ruiz Huidobro

Gerente de Exploración

Yacimientos Petrolíferos Fiscales

Avenida Rogue Seanz Pena 777

1364 Buenos Aires

Engineer: Dr. Enrique Mainardi

Comision Nacional de Investigaciones Espaciales (CNIE)

Dorrego 4018

1425 Buenos Aires

Fabricaciones Militares

Cabildo $65,2^{\circ}$ Piso

1426 Buenos Aires

Sub-Director de Desarrollo Minero

Cnel. J. Luis Cayetano Torres

Comision Nacional de Energía Atómica
Presidente: Brig. (RE) Guillermo Héctor Marotta

Avenida Libertador 8250 (1429)

Buenos Aires

President: Dr. Carlos Castro Madero
Yacimientos Carboníferos Fiscales

Avenida R. Saenz Pena $11902^{\circ}$ Piso 1035 Buenos Aires

Administratador General: Lic. Jose Antonio Iturriza

Instituto de Geocronología y Geología Isotopica Facultad de Ciencias Exactas y Naturales Ciudad Universitária Nunez Pabellon 2

1428 Buenos Aires

Director: Dr. Enrique Linares

Secretaría de Estado de Ciencia y Tecnología Cordoba 831

(1054) Buenos Aires

Secretary: Tcnl. Mario Antonio Remetin

Servicio de Hidrografía Naval

Montes de Oca 2124

(1271) Buenos Aires

Jefe: Cnel. Alberto José Valdes

Instituto Nacional de Ciencia y Técnica Hídricas

Viamonte 542

1053 Buenos Aires

Presidente: Com. (RE) Ing. Oscar Gregorio Velez

Instituto Geográfico Militar

Avenida Cabildo 301 (1426)

1426 Buenos Aires

Director: Cnel. Jorge Alberto Bavastro

$\mathbf{R}$

Secretaría de Estado de Minería

Av. Julio A. Roca 651 Piso $2^{\circ}$

1322 Buenos Aires

Secretary: Ing. Luis F. Gottheil

$\mathbf{R}$

Director Nacional de Combustibles

Secretaria de Estado de Energía

Avenida Julio A. Roca $6517^{\circ}$ Piso

1322 Buenos Aires

Director: Dr. Floréncio Pedro Cuervo

$\mathbf{R}$

Director Nacional Economía Minera

Avenida Santa $\mathrm{Fe} 1548,5^{\circ}$ Piso

(1060) Buenos Aires

Director: Dr. Pelayo Peñas 
AUSTRALIA

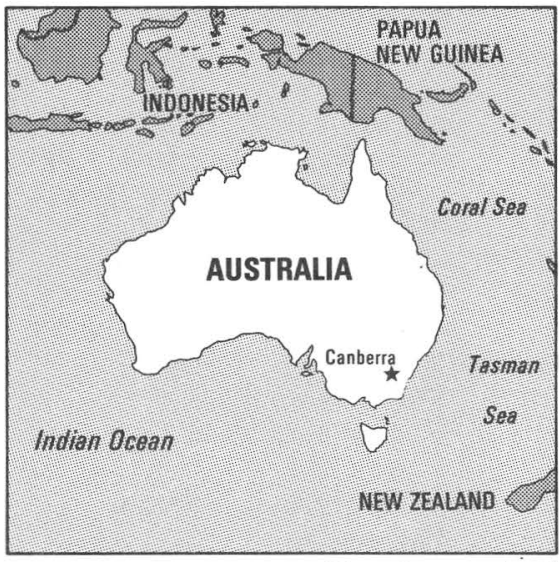

(See reference map $X$ )

G,H,C,R Department of Resources and Energy

G. P. O. Box 858

Canberra, City, A.C.T. 2601

Minister: Senator Peter Walsh

G,C

Bureau of Mineral Resources, Geology, and Geophysics

P.O. Box 378

Director: R. W. R. Rutland

G

G

National Energy Office

G.P.O. 858, Canberra City

A.C.T. 2601

Director: B. W. Fraser

Energy Policy Division

G.P.O. 858, Canberra City

A.C.T. 2601

First Assistant Secretary: E. W. C. Mathews

G

Regional Research and Development Division

G.P.O. 858, Canberra City

A.C.T. 2601

First Assistant Secretary: W. B. Rotsey

G

Uranium and General Division

G.P.O. 858, Canberra City

A.C.T. 2601

First Assistant Secretary: P. Ryan

G

G
Oil and Gas Division

G.P.O. 858, Canberra City

A.C.T. 2601

First Assistant Secretary: J. C. Starkey

G,H Institute of Energy and Earth Resources

Commonwealth Scientific and Industrial Research Organization

P.O. Box 225

Dickson, A.C.T., 2602

Director: I. E. Newnhan

H

Water and Development Division

Department of Resources and Energy

P.O. Box 858

Canberra City, A.C.T. 2601

First Assistant Secretary: A. Manderson

$\mathrm{H}$

Australian Water Resources Council

c/o Department of Resources and Energy

G.P.O. 858

Canberra, A.C.T. 2601

Secretary: J. A. Shaw

R

R

R

Department of Trade

Edmund Barton Bldg., Kings Avenue

Barton, A.C.T., 2600

Minister: Deputy Prime Minister Lionel Bowen and Minister for Trade

Australian Atomic Energy Commission

Private Mail Bag

Sutherland, N.S.W. 2232

Chairman: Professor M. H. Brennan

Joint Coal Board

G.P.O. 3842

Sydney, N.S.W. 2001

Chairman: G. J. Tredinnick 


\section{AUSTRIA}

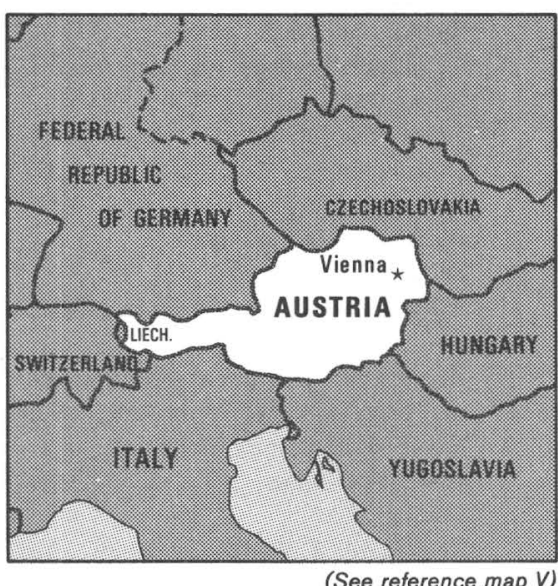

G

Geologische Bundesanstalt

Rasumof skygasse 23

A-1031 Vienna

Director: Prof. Dr. Traugott Gattinger

Bundesamt für Eich-und Vermessungwesen

Gruppe Landesaufnahme

Krotenthallergasse 3

A-1080 Vienna

President: Hofrat Dipl. Ing. Dr. Johann Bernhard

Bundesamt f. Eich-u. Vermessungswesen

Gruppe Kataster, Grundlagenvermessung

Staatsgrenzen

Fasangartengasse 101

A-1130 Vienna

Director: Hofrat Dipl. Ing. Hubert Höllinger

Osterreichische Kommission für die Internationale Erdmessung

Technische Universität Wien

Gusshausstrasse 27-29

A-1040 Vienna

President: Prof. Dipl. Ing. Dr. Karl Rinner, TU

Graz Rechbauerstrasse 12, A-8010 Graz

Secretary: Prof. Dipl. Ing. Dr. Kurt Bretterbauer

$\mathrm{H}$

Hydrographisches Zentralbuero

Bundesministerium für Land- und Forstwirtschaft

Marxergasse 2

A-1030 Vienna

Secretary: Dipl. Ing. Ernst Glasel

H,G Zentralanstalt für Meteorologie und Geodynamik

Hohe Warte 38

A-1190 Vienna

Director: Prof. Dr. Heinz Reuter

$\mathrm{R}, \mathrm{C}$

Bundesministerium füe Handel, Gewerbe und Industrie

Stubenring 1

A-1011 Vienna

Director: Dipl. Ing. Dr. Georg Sterk

\section{BAHAMAS, THE}

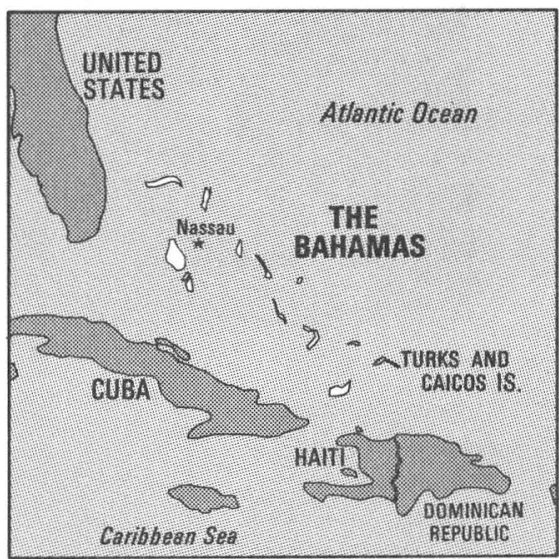

(See reference map III)

$\mathrm{H}$

C

$\mathrm{R}$

Minister of Economic Affairs

P.O. Box N-4596

Nassau

Minister: The Honorable Alfred T. Maycock 


\section{BANGLADESH}

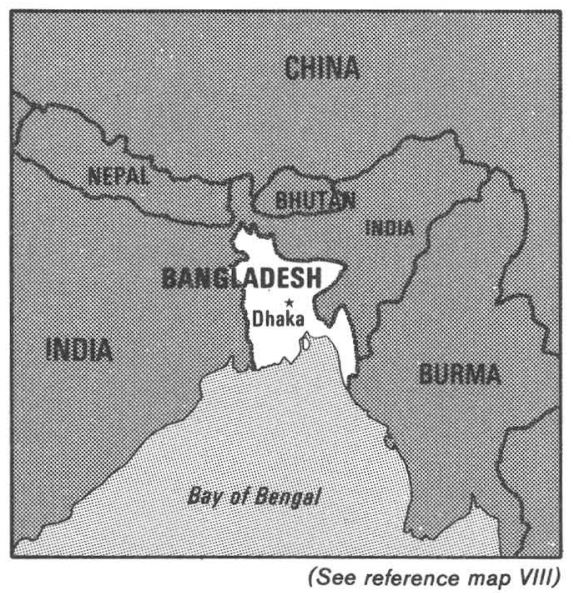

G

G

C

C

$\mathrm{H}$

$\mathrm{H}$

$\mathrm{H}$

Geological Survey

Pioneer Road

Segun Bagicha

Dhaka

Director General: M. Abu Bakr

Bangladesh Space Research and Remote Organization (SPARRSO)

Sher-e-Banglanagar

Agargaon, Taltola

Dhaka

Principle Investigator: Dr. Faruk A. Khan

Land Records and Survey Department

Tejgaon Industrial Area

Dhaka

Director: Abdul Bari Tarafder

Survey of Bangladesh

Tejgaon Industrial Area

Dhaka

Director General: M. Hafizuddin

Ground Water Circle

Bangladesh Water Development Board

48 Motijheel Commercial Area

Dhaka

Director: Gulam Mawla

Surface Water Hydrology-I

Bangladesh Water Development Board

72 Green Road

Dhaka

Director: Abdul Matin

Surface Water Hydrology-II
$\mathbf{R}$

$\mathbf{R}$

\section{BARBADOS}

G

Bangladesh Water Development Board

72 Green Road

Dhaka

Director: A. Wazed
Petro-Bangla

[Bangladesh Oil and Gas Development Corp.]

122-124 Motijheel Commercial Area

Dhaka

Chairman: Group Capt. Kazi Aftabuddin Ahmed

Ministry of Energy and Mineral Resources

[Petroleum and Mineral Resources Division]

Main Building (1st Floor)

Bangladesh Secretariat

Dhaka

Additional Secretary In-Charge: Shafiul Alam

Bangladesh Mineral Exploration and

Development Corporation (BMEDC)

H.B.F.C. Building (9th Floor)

22 Purana Paltan

Dhaka

Chairman: M. W. Ali

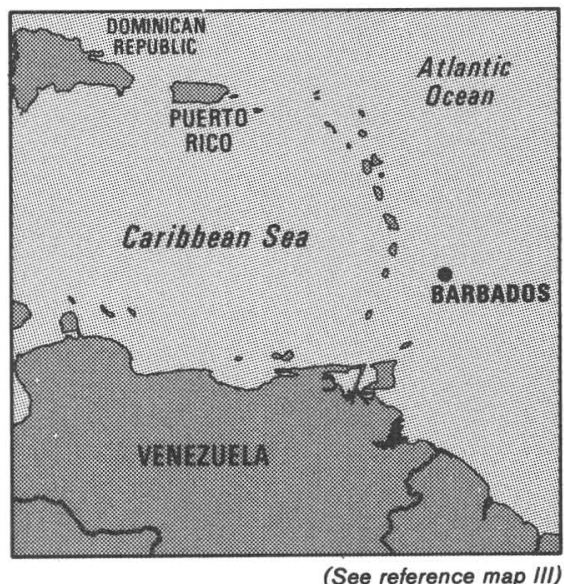

(See reference map III)

Ministry of Finance and Planning

Governmental Headquarters

St. Michael

Chief Geologist: L. H. Barker 


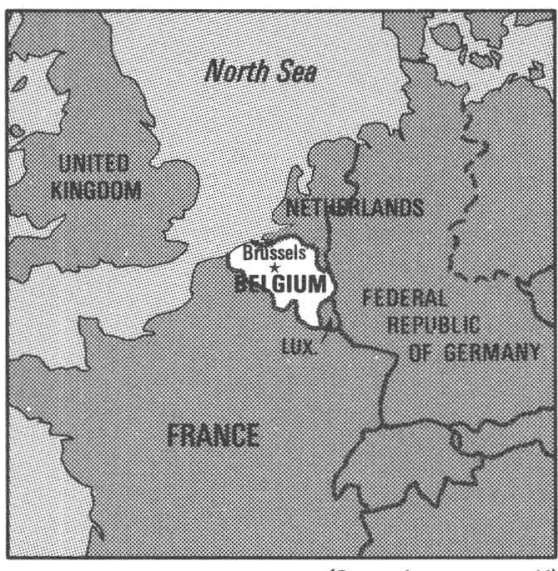

(See reference map V)

G

G 1050 Brussels

Director General: R. Verberckt

Ministère des Travaux Publiques

Service de Topographie et de Photogrammetrie 155 Rue de La Loi

1040 Brussels

Chief Engineer: R. De Fays

Ministère des Affaires Economiques

Service Hydrologique

30 Rue J.A. De Mot

1040 Brussels

Inspector General: H. Gregoire

Ministère des Travaux Publiques

Administration des Voies Hydrauliques

155 Rue de La Loi

1040 Brussels

Director General: J. Demoen

Ministère des Affaires Economiques
R

Comité National de l'Energie

6 Rue de L'Industrie

1040 Brussels

Secretary General: H. Bernard

H

Association Nationale des Services d'Eau

(ANSEAU)

255 B. 6 Chaussee de Waterloo

1060 Brussels

President: M. Snel

\section{BELIZE}

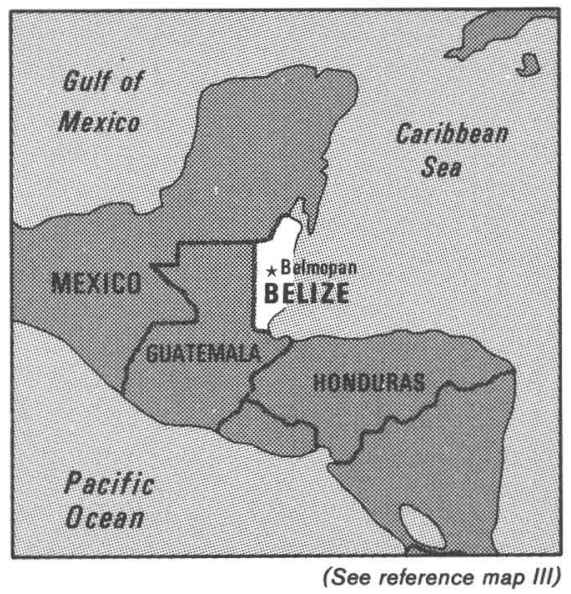

G

Administration de l'Energie

30 Rue J.A. De Mot

1040 Brussels

Director General: M. Frerotte

Inspector General for Coal: F. Sonck

Chief Engineer for Petroleum-Gas: J. Hots 
(No recent information)

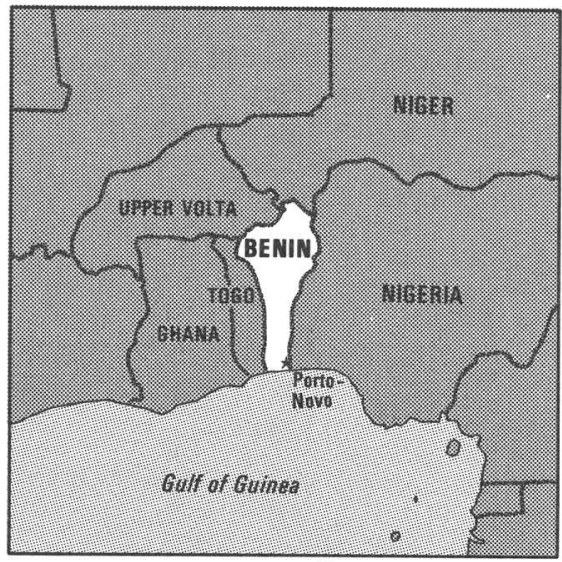

(See reference map VII)

G

G,H, Office de la Recherche Scientifique et Technique Outre-mer (ORSTOM) ${ }^{1}$

Centre de Contonou

B.P. 390

Cotonou

G

G

G

$\mathrm{H}$

\footnotetext{
${ }^{1}$ French research organization; no governmental affiliation.
}

Ministère de l'Equipement

B.P. 360

Cotonou

\section{BHUTAN}

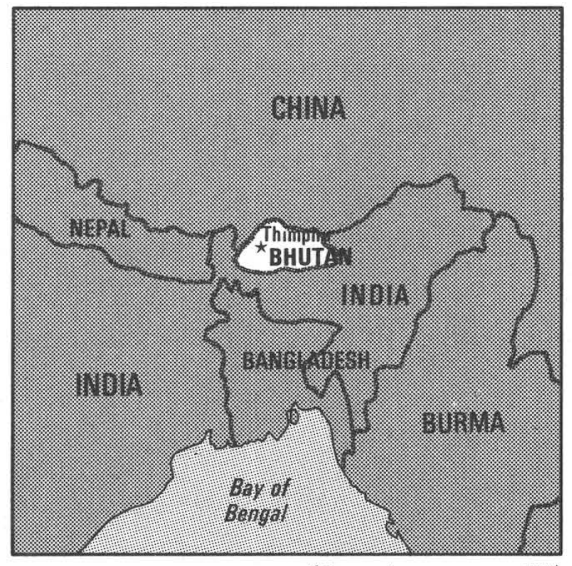

(See reference map VIII)

$\mathrm{G}, \mathrm{C}, \mathrm{H}$

Department of Trade and Industries

Thimphu

Joint Director: Ygyen Namgyel

\section{BOLIVIA}

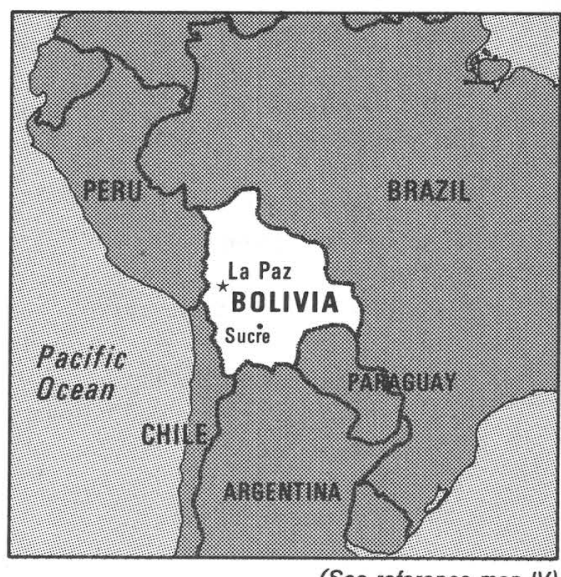

(See reference map IV)

G.H,C

Servicio Geológico de Bolivia (GEOBOL)

Federico Zuazo, Esq. Reyes Ortiz

Casilla de Correo 2729

La Paz

Director: Oscar Ballivian

G,C Centro de Investigacion y Applicacion de Sensores Remotes (CIASER)

Federico Zuazo, Esq. Reyes Ortiz

Casilla de Correo 2729

La Paz

Director of Department: Rene Valenzuela Rivero

Fondo Nacional de Exploración Minera (FNEM)

Calle Cordero 257

Casilla De Correo 5796

$\mathrm{LaPaz}$

General Manager: Abelardo Villalpando 
Observatorio San Calixto

Calle Jenaro Sanjinez 701

Casilla De Correo 5939

La Paz

Director: R. P. Ramon Cabre

G

Empresa Siderúrgica Boliviana (SIDERSA)

Avenida 16 de Julio No. 1769

La Paz

Casilla de Correo 7082

General Manager: Rene Rengel-Dominguez

G

Corporación Minera de Bolivia (COMIBOL)

Avenida Mariscal Santa Cruz 1092

Casilla de Correo 349

La Paz

General Manager: Oscar Torrico-Pedrazas

Technical Manager: Rene Saenz-Molina

G, R Yacimientos Petrolíferos Fiscales Bolivianos (YPFB)

Calle Bueno 185

Casilla de Correo 401

$\mathrm{LaPaz}$

General Manager: Carlos Uría

Exploration Manager: Vicet Ponce de Leon

C Instituto Geográfico Militar

Avenida Saavedra, Cuartel Miraflores

$\mathrm{LaPaz}$

Director: Hernan Alfaro-Cortez

H Oficina de Aguas Potables por Communidades

La Paz

Chief: P. A. Parada P.

Director General: Mario Canedo-Daza

Ministerio de Minería y Metalurgia

Avenida 16 de Julio 1769

La Paz

Casilla de Correo 8686

Minister: Carlos Carvajal Nava

Instituto de Investigaciones Minero-Metalúrgico (IIMM)

Calle Junin 1037

Casilla de Correo 600

Oruro

Director: Mario Paulsen

Ministerio de Energía e Hidrocarburos

Avenida Mariscal Santa Cruz 1322

Casilla de Correo 4819

La Paz

Minister: Luis Sauedo Justiniano
C

R

$\mathrm{G}, \mathrm{R}$ Comisión Boliviana de Energía Nuclear (COBOEN)

Calle 6 de Agosto 2905

Casilla de Correo 4821

La Paz

Director: Antonio Rondon

H,C Servicio de Hidrografía Naval

Avenida Argentina 2057

Casilla de Correo 3052

La Paz

Director: Alberto Saenz-Klinsky

\section{BOTSWANA}

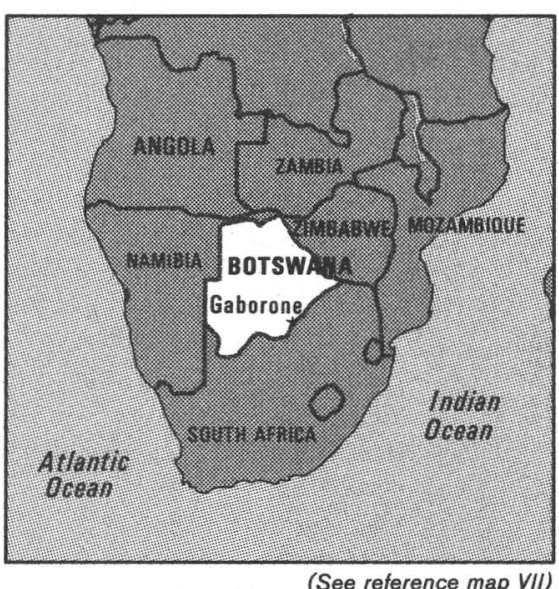

(See reference map VII)

Geological Survey Department

Ministry of Mineral Resources and Water Affairs

Private Bag 14

Lobatse

Director: Dr. G. C. Clark

Department of Surveys and Lands

Ministry of Local Government and Lands

Private Bag 37

Gaborone

Director: W. L. Dickson

$\mathrm{H}$

Department of Water Affairs

Ministry of Mineral Resources and Water Affairs

P.B. 29

Gaborone

Director: G. M. Quraishi

Department of Mines

Ministry of Mineral Resources and Water Affairs

Private Bag 49

Gabarone

Mining Commissioner: Peter Cain 
Ministry of Mineral Resources and Water Affairs

Private Bag 29

Gaborone

Permanent Secretary (all departments): Charles Tibone

\section{BRAZIL}

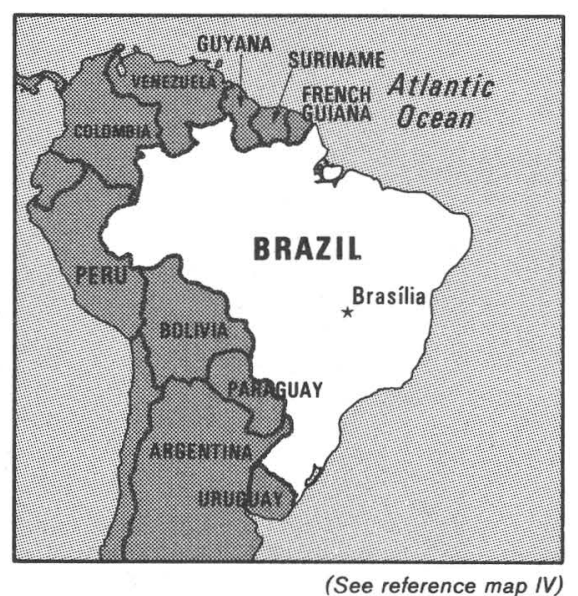

G,C Instituto de Pesquisas Espaciais (INPE)

Caixa Postal 515

12200 São José dos Campos

São Paulo, SP

Director General: Nelson de Jesus Parada

G

Companhia de Pesquisa de Recursos Minerais (CPRM)

Avenida Pasteur, 404 - Anexo, $2^{\circ}$ Andar,

Praia Vermelha

22290 Rio de Janeiro, RJ

President: General Salvador

Goncalves Mandim

G

Rio Doce Geológica e Mineracao S/A (DOCEGEO)

Companhia Vale do Rio Doce (CVRD)

Av. Presidente Wilson $210,11^{\circ}$ Andar

22030 Rio de Janeiro, RJ

President CVRD: Eliezer Batista

Director-Superintendent of DOCEGEO:

Francisco de Assis Fonseca

G,R Petroleo Brasileiro S.A. (PETROBRAS)

Avenida Republica do Chile, 65, $22^{\circ}$ Andar

20031 Rio de Janeiro, RJ

President: Shigeaki Ueki

G, R Departamento Nacional de Producao Mineral (DNPM)

SAN - Q 1, Bloco B, $3^{\circ}$ Andar, Sala 308

70040 Brasilia, D.F.

Director General: Yvan Barretto de Carvalho
G,R Empresas Nucleares Brasileiras S.A. (NUCLEBRAS)

SAN - Bloco D - $8^{\circ}$ Andar

70040 Brasilia, DF

President: Dario Jose Goncalves Gomes

Director of Mineral Exploration: John M. A. Forman Av. Presidente Wilson, 231, $22^{\circ}$ Andar 20030 Rio de Janeiro, RJ

H

C

C

H

C

$\mathbf{R}$
Departamento Nacional de Obras de Saneamento (DNOS)

Av. Pres. Vargas $62,11^{\circ}$ Andar

20091 Rio de Janeiro, RJ

Director General: José Reinaldo Carneiro Tavares WMO/CHy Representative: Paulo Poggi Periera

Diretoria do Servico Geográfico do Exército (DSG) SMU - QG Ex., Bloco F, $2^{\circ}$ Pav. 70630 Brasilia, D.F.

Director: General Aristides Barreto

Diretoria de Geodesia e Cartografia Instituto Brasileiro de Geografia e Estatística (IBGE) Avenida Brasil, 15015 21241 Rio de Janeiro, RJ Director: Mauro Pereira de Mello

Departmento Nacional de Aguas e Energia Eletrica (DNAEE)

Ministerio das Minas e Energia Esplanada dos Ministérios Bloco J, $3^{\circ}$ Andar, Sala 300 70056 Brasilia, D.F.

Director General: Alvarino de Araujo Pereira

Projeto RADAM

Av. Antonio Carlos Magalhaes, 1131

Edf. Marechal Ademar de Queiroz, $4^{\circ}$ Andar

40000 Salvador, BA

Executive Secretary: Antonio Luiz Sampaio de Almeida

Ministry of Mines and Energy Esplanada dos Ministerios-Bloco J, $8^{\circ}$ Andar 70056 Brasilia, D.F.

Minister: Cesar Cals de Oliveira Filho

Conselho Nacional do Petroleo (CNP)

Ministerio das Minas e Energia

SGAN - Q. 603 Modulos J, I e H

70830 Brasilia, DF

President: General Oziel Almeida Costa 
$\mathrm{R}, \mathrm{G}$

Comissão Nacional de Energia Nuclear (CNEN)

Rua General Severiano, 90

Botafogo

22290 Rio de Janeiro, RJ

President: Rex Nazare Alves

Department of Mineral Resources (CNEN):

Carlos Pires Ferreira

\section{BRUNEI}

(No recent information)

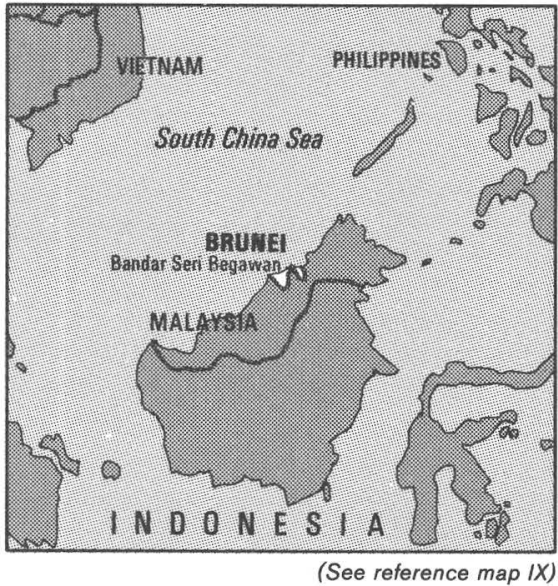

G

C

The Government Geologist

Bandar Seri Begawan

Survey Department of Brunei

Bandar Seri Begawan

\section{BULGARIA}

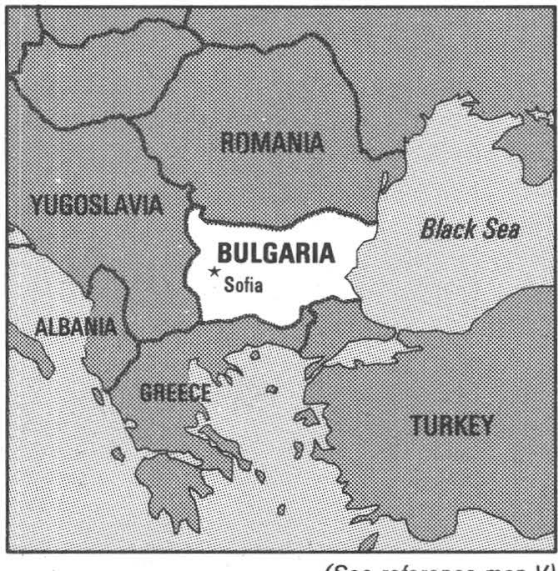

G
G, R

G

G

G

G

G

G

G

G
Research Institute for Mineral Resources

23, Chr. Kabakchiev Blvd.

Sofia 1505

Director: Dr. Chr. Datchev

Laboratory of Experimental and Technical Mineralogy at the Bulgarian Academy of Sciences

6, Moskovska Street

Sofia 1000

Director: Prof. Mihail Maleev

Committee of Geology

22, G. Dimitrov Blvd.

Sofia 1000

President: Dr. Rodion Dokov

Ministry of the Energy and Raw Material Resources

22, G. Dimitrov Blvd.

Sofia 1000

Minister: Dr. Todor Bojinov

Centre for Earth Sciences at the Bulgarian Academy of Sciences

Acad. G. Bonchev Street, Block 3

P.O. Box 134,

Sofia 1113

Director: Corr. Member, Prof. Kiril Mishev

Bulgarian Academy of Sciences

Institute of Geography

Acad. G. Bonchev Street, Block 3,

Sofia 1113

Director: Corr. Member, Prof. Kiril Mishev

Bulgarian Academy of Sciences

Geophysical Institute

Acad. G. Bonchev Street, Block 3,

Sofia 1113

Director: Dr. Dimitar Samardjiev

Bulgarian Academy of Sciences

Geotechnical Laboratory

Acad. G. Bonchev Street, Block 24

Sofia 1113

Director: Prof. Minko Minkov

National Oceanographic Committee

8 , Slavijanska Street

Sofia 1000

Bulgarian Academy of Sciences

Institute of Marine Research and Oceanology

P. Box 152

Varna 9000

Director: Dr. Zdravko Belberov 
Central Laboratory for Space Research

6, Moskovska Street

Sofia 1000

Director: Corr. Member, Prof. Kiril Serafimov

Bulgarian Academy of Sciences

Central Laboratory for Geodesy

1, 7 Noemvri Street

Sofia 1000

Director: Prof. Dr. Nikola Georgiev

$\mathrm{H}$

Bulgarian Academy of Sciences

Institute of Waterproblems

Acad. G. Bonchev Street, Block 3

Sofia 1113

Director: Prof. Peter Ignatov

\section{BURMA}

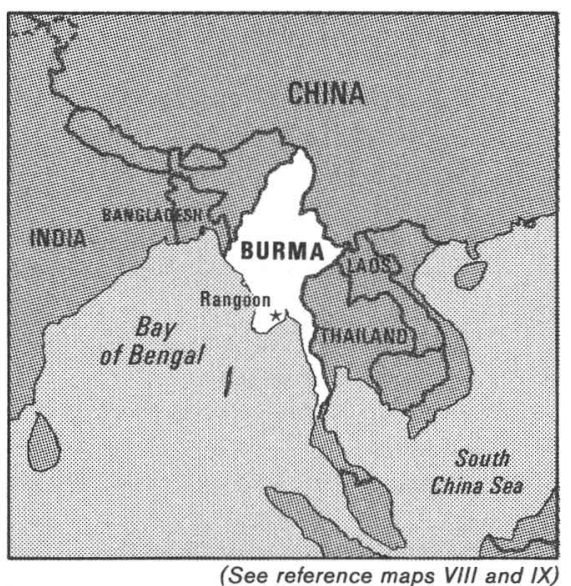

G

Department of Geological Survey and Exploration

Ministry of Mines

Kanbe Road, Yankin P.O.

Rangoon

Managing Director: Col. Aung Din

G

Applied Geology Department

6-1/2 Mile Prome Road

Rangoon

Director: Dr. Myint Lwin

G

Myanma Oil Corporation

604 Merchant Street

Rangoon

Managing Director: U Than Nyunt

G

No. (1) Mining Corporation
G

No. (2) Mining Corporation

Kanbe Road, Yankin P.O.

Rangoon

Managing Director: Lt. Col. Maung Thaung

No. (3) Mining Corporation

Rangoon-Insein Road, Thamaing

Rangoon

Managing Director: U San Maung

H

Irrigation Department

9/21 Strand Road

Ministry of Agriculture and Forestry

Rangoon

Director General: U Maung Maung Kyi

$\mathrm{H}$

Directorate of Meteorology and Hydrology

Kaba Aye Pagoda Road

Rangoon

Director General: U Thu Ta

C

Central Research Organization

Kanbe Road, Yankin P.O.

Rangoon

Director General: Col. Oo Saw Hla

C

\section{Survey Department}

Ministry of Agriculture and Forestry

Thirimingala Lana

Kaba Aye Pagoda Road

Rangoon

Director General: U Lun Pe

\section{BURUNDI}

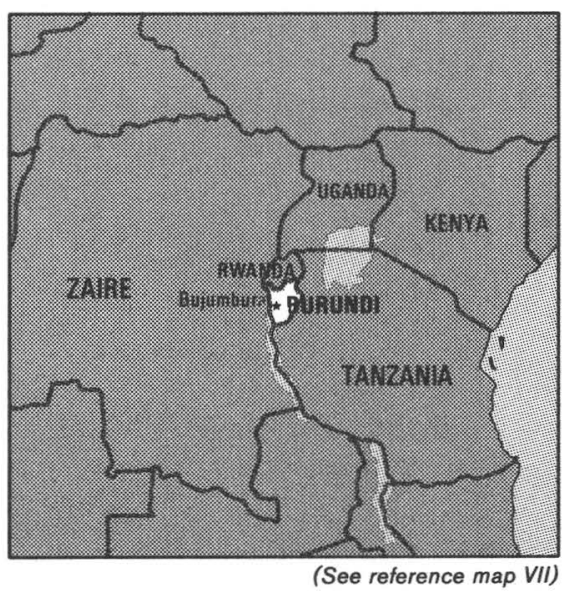

104 Strand Road

Rangoon

Managing Director: U Ko Ko Than 


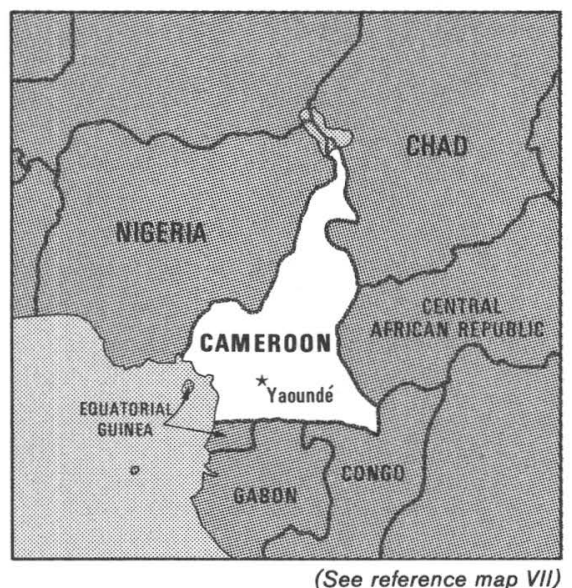

G

G

G

G,C,H Institut de Recherches Géologiques et Minières (IRGM)

B.P. 4110

Yaoundé

Director: Soba Djallo

Chief Hydrology Service: Emmanuel Naah

C Centre Géographique National

B.P. 157

Ave. Monseigneur Vogt

Yaoundé

Director: Daniel Schamukong

$\mathrm{C}, \mathrm{H} \quad$ Institut des Recherches Agricoles et Forestieres (IRAF)

B.P. 2123

Yaoundé

Director: Anthony Maimo

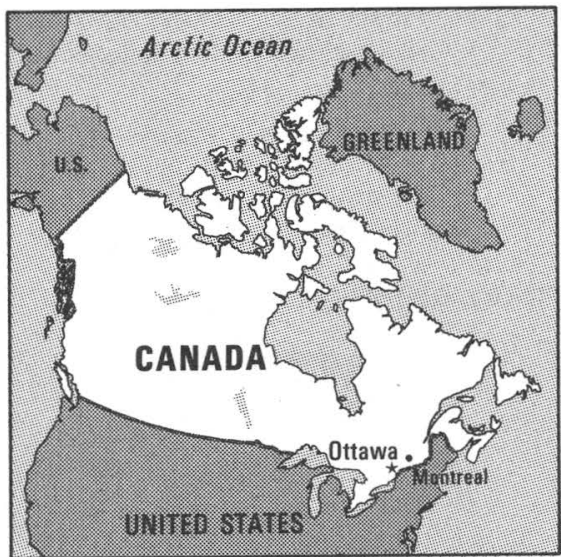

(See reference map II)

G

G

\footnotetext{
${ }^{2}$ French research organization; no governmental affiliation.
} 
Department of Energy, Mines, and Resources 580 Booth Street

Ottawa, Ontario, K1A 0E4

Senior Assistant Deputy Minister: L. Good

R,H Northern Affairs Program

Department of Indian and Northern Affairs

Les Ferrasses de la Chaudiere

Ottawa, Ontario KIA OH4

Assistant Deputy Minister: G. N. Faulkner

\section{CENTRAL AFRICAN REPUBLIC}

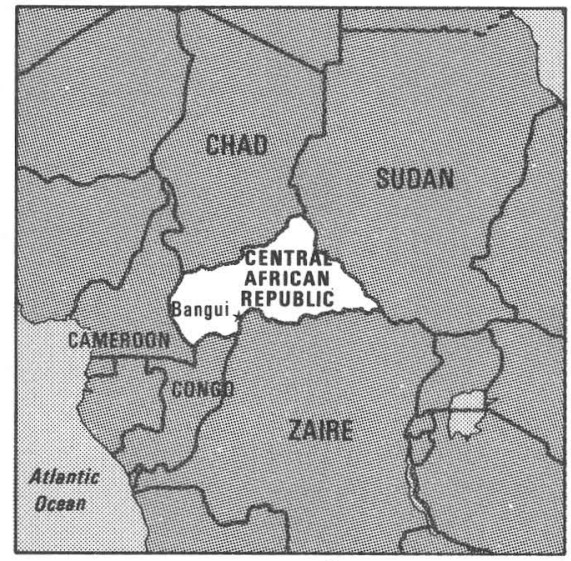

(See reference map VII)

G

Ministry of Water, Lands, Games Fishing, and Tourism

Bangui

Minister: Lieutenant-Colonel Sēbastien Guipi

G,C Ministry of Public Works and Town Development Bangui

Minister: Brigadier General Abel Nado

G, R,H,C Office de la Recherche Scientifique et Technique Outre-mer (ORSTOM), Centre de Bangui $^{3}$ B.P. 893

Bangui

Directeur: Jean-Paul Cointepas

G,H Ministry of Energy and Hydraulics

Bangui

State Minister: Lieutenant-Colonel Thomas Mapouka

G Ministry of Agriculture and Animal Husbandry Bangui

State Minister: Lieutenant-Colonel

Alphonse Gombadi

G, R High Commission for Mining and Geology

Bangui

High Commissioner:. Dr. Jean Euded Téya

C, G, R,H High Commission for Scientific and Technological Research

Bangui

High Commissioner: Jean-Claude Kazagui

C Institut National Géographique (ING)

B.P. 165

Bangui

Representative: Jean-Pierre Torrès
CHAD

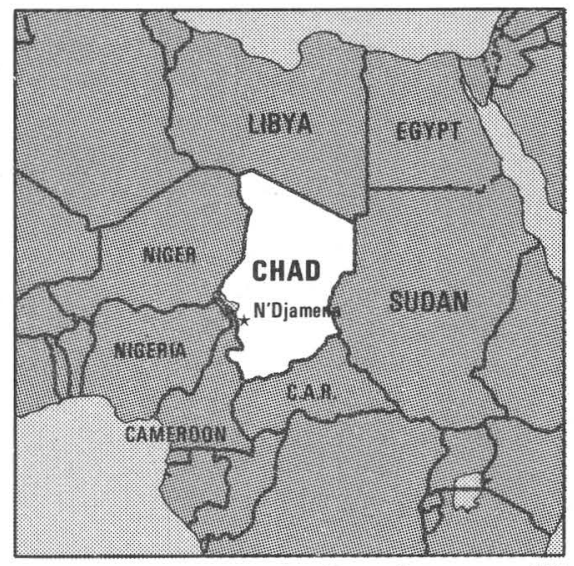

(See reference map VII)

G,C,R

Direction des Mines et Pétrole

B.P. 816

N'Djamena

Director: Eugène Tabe

C,H Direction du Génie Rural

B.P. 47

N'Djamena

Director: Abdéramane Abdoul

H

Office National de l'Hydraulique Pastorale

B.P. 750

N'Djamena

Director: Tahar Sougoudi Sidimi

\section{CHILE}

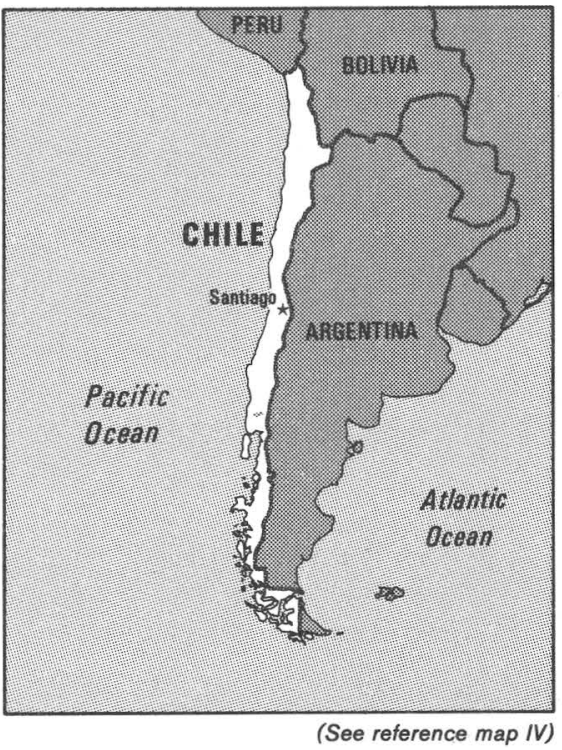

G

Dirección de Geología

Corporación Nacional del Cobre

Huérfanos $1189,8^{\circ}$ Piso

Santiago

Director: Héctor Flores Williams 
Centro de Investigaciones Minero-Metalúrgicas

Parque Institucional 6500, Lo Curro

Santiago

Director: Werner Schlein

G

Instituto de Investigación de Recursos Naturales (IREN)

Corporación de Fomento de la Producción

Manuel Montt 1164

Santiago

Executive Director: Enrique Junemann Mardones

G

Departamento Nacional de Geología

Empresa Nacional del Petróleo

Ahumada $341,8^{\circ}$ Piso

Santiago

Chief, Eduardo González Pacheco

Servicio Nacional de Geología y Minería (SERNAGEOMIN)

Teatinos 120, Piso 9

Santiago

National Director: María Teresa Cañas

Sub-Director of Geology: Carlos Ulriksen

Sub-Director of Mines: Juan Divin

Unidad de Minería

Corporación de Fomento de la Producción

Moneda 921, Of. 538

Santiago

Chief: Juan Montoya

Comisión Chilena de Energía Nuclear (CCEN)

Los Jesuítas 645

Casilla 188-D

Santiago

Director: Teniente General (R) Herman Brady Roche

$\mathrm{H}$

Empresa Nacional de Electricidad S.A.

Casilla 1392

Santiago

WMO/CHy Representative: Andrés Benitez

H

Instituto Hidrográfico de la Armada

Errazuriz 232

Casilla 324

Valparaíso

Captain Eduardo Barison Roberts

C

Servicio Aerofotogramétrico

Fuerza Aérea de Chile

Casilla 67

Correo Los Cerrillos

Santiago

Director: Comandante de Grupo del Aire Oscar Boronig S.

C

Instituto Geográfico Militar

Nueva Santa Isabel 1640

Santiago

Director: Enrique López Silva
R

Ministerio de Minas

Teatinos 120

Santiago

Minister: Samuel Lira Ovalle

\section{CHINA ${ }^{4}$}

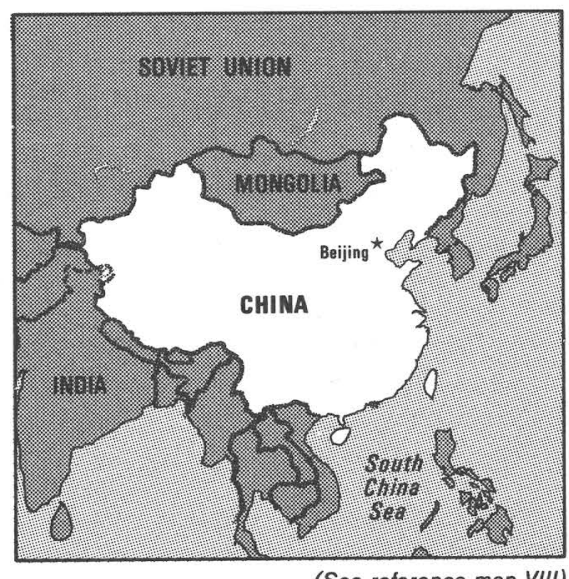

(See reference map VIII)

G

Academia Sinica

[Chinese Academy of Science]

Division of Earth Sciences

Vice President: Ye Duzheng

The Academy consists of the following Institutes:

G

Desert Studies

Lanzhou

Director:. Zhu Zhenda

G

Geochemistry

Guiyang

Director: Tu Guangzhi

G

Geography

Beijing

Director: Huang Bingwei

G

Geography

Changchun

Director: Huang Xichou

G

Geography

Chengdu

Director: Ding Xizhi

G

Geography

Xinjiang

Director: Yue Zhen

G

Geology

Beijing

Director: Zhang Wenyou

${ }^{4}$ See Taiwan, p. 96. 
G

G

G

G

G

G

G

G

G

C

G

G

C

H

G

G
Geology

Lanzhou

Director: Huang Ruchang

G

Geology and Geography

Urumchi

Geology and Paleontology

Nanjing

Director: Zhao Jinke

Geophysics

Beijing

Director: Fu Chengyi

Geotectonics

Changsha

Director: Chen Guoda

Glaciology and Cryopedology

Lanzhou

Director: Shi Yafeng

Oceanography

Qingdao

Director: Zeng Chengkui

Oceanography (South Seas)

Guangzhou

Director: Qiu Bingjing

Pedology

Nanjing

Director: Xiong Yi

Remote Sensing Application

Beijing

Chen Shuping

Rock and Soil Mechanics

Wuhan

Salt Lakes

Xining

Director: Liu Dagang

Surveying

Wuhan

Director: Fang Jui

Soil and Water Conservation

Shaanxi

Director: Zhu Xianmo

Vertebrate Paleontology of Paleoanthropology

Beijing

Director: Zhou Minzhen

Ministry of Geology and Mineral Resources

(Formerly known as the State Bureau of Geology)

Minister: Sun Daguang
G

G

G

G

G

G

G

$\mathrm{H}$

G

G

G

G

G

The Ministry consists of the following units:

Academy of Geological Sciences

Beijing

Director: Li Tingdong

Composite Geology Research Team

Shanghuxian

Director: Ma Shuzhao

Institute of Geology

Beijing

Director: Shen Qihan

Institute of Geology of Mineral Deposits

Beijing

Director:. Chen Yuchuen

Institute of Geology of Plateau Areas

Chengdu

Director: Liu Zengqian

Institute of Geologic Information

Beijing

Director: Feng Zhongguang

Institute of Geomechanics

Beijing

Director: Sun Dianqing

Institute of Hydrogeology and Engineering Geology

Zhengding

Director: Zhang Zoughu

Institute of Karst

Guilin

Director: Yuan Daoxian

Institute of Geology and Mineral Resources (Sedimentary Rocks)

Chengdu

Director: Liu Baojun

Institute of Geology and Mineral Resources (Volcanic Rocks)

Nanjing

Director: Ye Zhizeng

Institute of Geology and Mineral Resources Shenyang

Director:. Qin Nai

Institute of Geology and Mineral Resources (Quaternary and Precambrian)

Tianjin

Director: Shen Baofeng

Institute of Geology and Mineral Resources Xian

Director: Li Xianxi 
Institute of Geology and Mineral Resources Yichang

Director: Tan Zhangfu

Institute of Rock and Mineral Analysis

Beijing

Director: Wang Weiying

National Geological Library

Beijing

Director: Deng Geming

National Geological Museum

Beijing

Director:. Liu Yongquan

Institute of Multipurpose Utilization of Mineral Resources

Emei

Director: Zheng Youlang

State Seismological Bureau

Beijing

Director: An Qiyuan

Institute of Seismology

Wuhan

Director: Lee

Institute of Geophysics

Beijing

Director: Cheng Zongji

Institute of Geology

Beijing

Director: Ma Xingyuan

Institute of Engineering Mechanics

Harbin

Director: He Lungjiang

Ministry of Coal Industry

Beijing

Minister: Gao Yanquen

Institute of Coal Geology and Exploration Xian

Director: Yuan Yaoting

National Bureau of Oceanography

Beijing

National Bureau of Surveying and Cartography Beijing

Ministry of Petroleum Industry

Beijing

Minister: Tang $\mathrm{Ke}$

Institute of Petroleum Exploration and

Beijing Development

Director: Shen Lisheng
G

Ministry of Nuclear Industry

Beijing

Minister: Jiang Xinxiong

G

Institute of Uranium Geology

Beijing

$\mathrm{H}$

Ministry of Water Resources and Electric Power Beijing

Minister: Qian Zhengying

Bureau of Hydrology

Beijing

Director: Chen Jiaqi

\section{COLOMBIA}

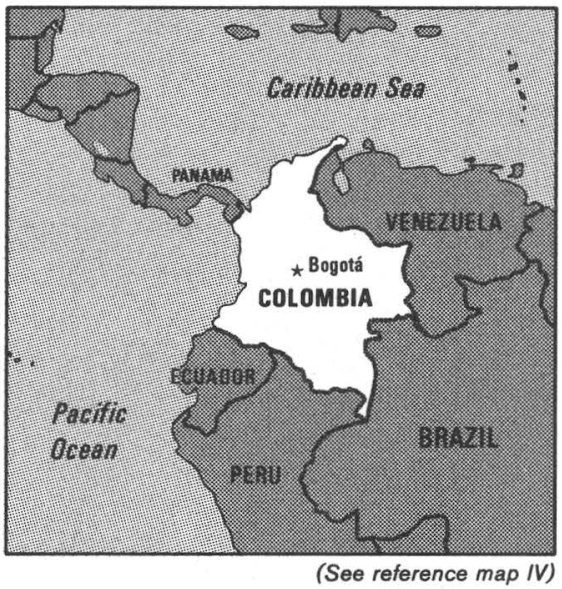

$\mathrm{G}, \mathrm{H}, \mathbf{R}$ Instituto Nacional de Investigaciones Geológico Mineras (INGEOMINAS)

Diagonal 53 \# 34-53

Apartado Aéreo 4865

Bogotá, D. E.

Director: Dr. Alfonso Lopez-Reina

G,H Instituto Colombiano de Hidrología, Meteorología y Adecuacion de Tierras (HIMAT)

Carrera 10 \# 20-19, Pisos 4 al 12

Bogotá, D.E.

Director: Dr. Fabio Bermúdez-Gómez, Oficina 1201

G

Instituto Geofísico de Los Andes

Carrera 7 \# 40-76, Universidad Javeriana

Apartado Aéreo 56710

Bogotá, D.E.

Director: Reverendo Padre Jose Rafael-Goberna

C Instituto Geográfico "Agustín Codazzi"

Carrera 30 \# 48-51

Apartado Aéreo 6721

Bogotá, D.E.

Director: Dr. Alvaro González-Fletcher 
Dirrección Mariitima y Portuaria-Armada Nacional

Ministerio de Defensa Nacional

CAN

Bogotá, D.E.

Apartado Aéreo 20294

Director: Contra Almirante Juan Pablo ReiránHernández

$\mathrm{C}$

Centro Interamericano de Fotointerpretación Carrera 30 \# 47A-57

Bogotá, D.E.

Director: Dr. Hernán Riviera-Hermida

R

Instituto de Asuntos Nucleares

IAN

Avenida Eldorado, Carrera 50

Apartado Aéreo 8595

Bogotá, D. E.

Director: Dr. Ernesto Villarreal

\section{CONGO}

(No recent information)

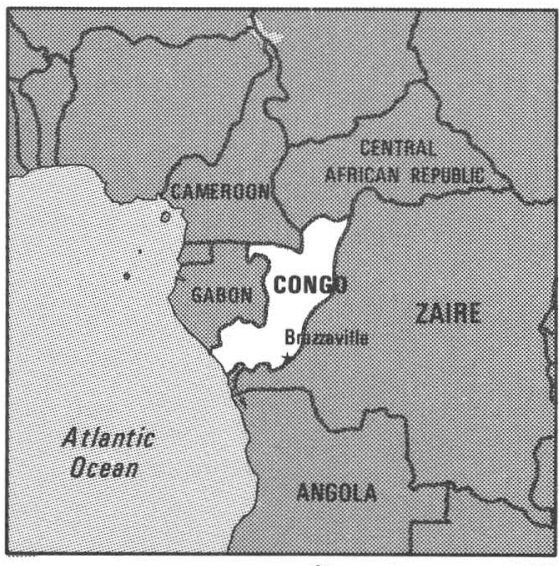

(See reference map VII)

G

Office de la Recherche Scientifique et Technique Outre-mer (ORSTOM) ${ }^{5}$

Centre Oceanographique de Point-Noire

B.P. 1286

Point Noire

Director: Fred Gueredrat

G Service des Mines et de la Géologie

B.P. 2124

Brazzaville

H Service Hydrologique de l'ORSTOM ${ }^{5}$

B.P. 181

Brazzaville

C Institut Géographique ${ }^{5}$

B.P. 125

Brazzaville

${ }^{5}$ French research organization; no governmental affiliation.
C

Cadastre du Congo

B.P. 544

Brazzaville

C

Service Topographique et du Cadastre

Agence de Brazzaville

B.P. 125 Route du Djove

Brazzaville

Director: Antoine Ondema

\section{COOK ISLANDS}

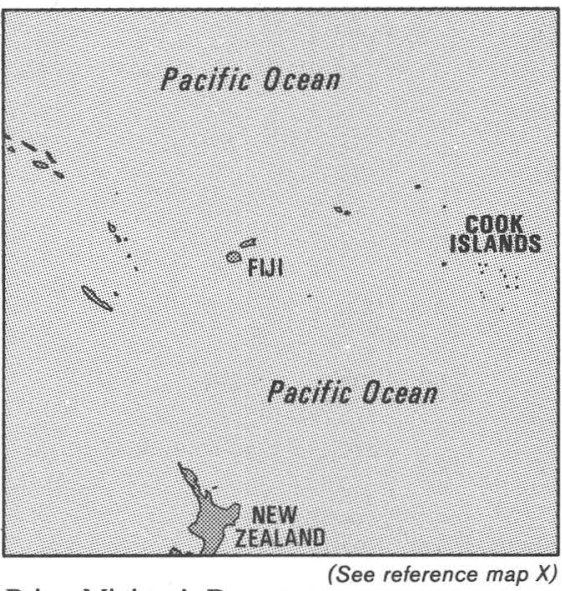

G Prime Minister's Department

P.O. Box 66

Raratonga

Scientific Research Officer: S. Kingan

G,C Department of Survey and Physical Planning Secretary of Survey and Physical Planning

Raratonga

Secretary: G. Cowan

\section{COSTA RICA}

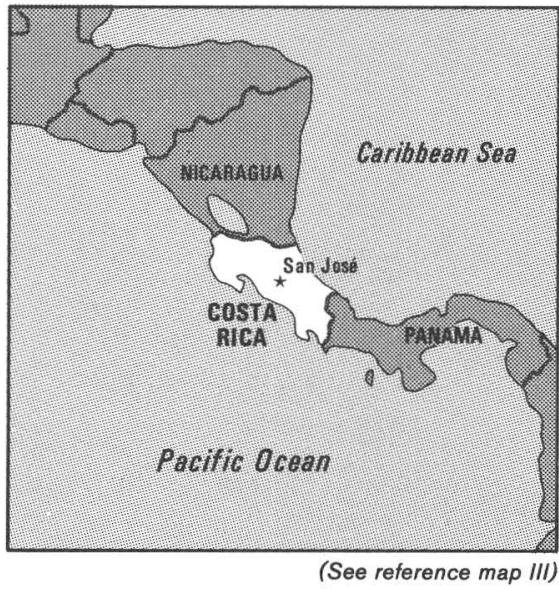

Departamento de Geología

Instituto Costarricense de Electricidad (ICE)

Apartado 10032, 1000 San José

Chief: Jorge E. Umaña 
Corporacion Costarricense de Desarrollo

$$
\text { (CODESA) }
$$

Apartado 10254, 1000 San José

Director: Dr. Alfonso Monge

Consejo Nacional de Investigaciones Científicas y Technológicas (CONICIT)

Apartado 10318, 1000 San José

President: Rodrigo Zeledon

Executive Secretary: Jose Martin Solozano

$\mathrm{C}, \mathrm{G} \quad$ Instituto Geográfico Nacional

Apartado 22721000 San José

Director: Fernando M. Rudin Rodriguez

$\mathrm{H}$

Departamento de Estudios Basicos

Instituto Costarricense de Electricidad (ICE)

Apartado 100321000 San José

Chief: Enrique Evans

H Servicio Nacional de Aguas Subterráneas (SENAS)

Apartado 52621000 San Jose

Executive Director: Alvaro Suarez

R

Dirección de Programación y Control de Exploración Petróleo

Refinadora Contarricense de Petróleo S.A. (RECOPE)

Apartado 43511000 San José

Director: Umberto Porta

Manager: Pedro Afonso

R,G Dirección de Minas, Geología, y Hidrocarburos Ministry of Energy, Mines, and Industry

Apartado 47521000 San José

Director: Jorge Vargas

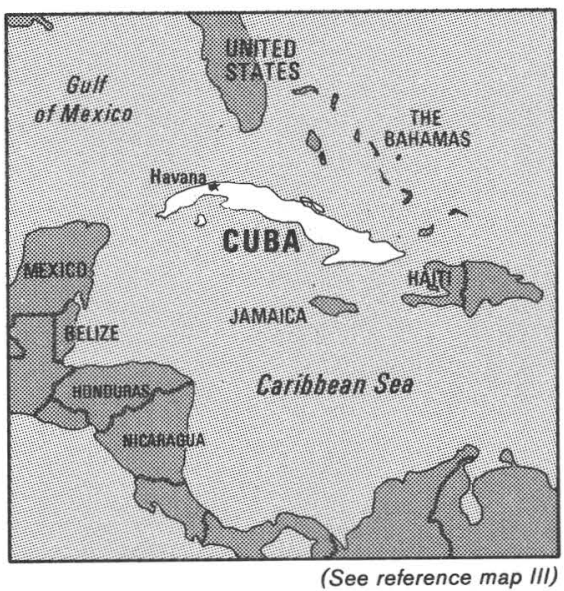

G

Instituto de Geología

Academia de Ciencias de Cuba

Ave. Van-Troi no. 17203

Rancho Boyeros-Apartado Postal 10

La Habana

Director: Jesus F. de Albear Franquiz

G

Instituto Nacional de Recursos Minerales (ICRM)

O'Reilly y Aguacate

La Habana

Director: Salvador Salas

Instituto Cubano de Geodesia y Cartografía

Loma y 39

Nuevo Vedado

La Habana

Director: Emilio Lluis Rojoo

G

Instituto de Geofísica y Astronomía

Calle 212, No. 2906

Reparto Cubánacan

La Habana 16

Director: Rosendo Alvares Morales

G

Instituto de Geología y Paleontología

Calzada No. 851

La Habana 4

Director: Lenia Montero Zamora

G

Instituto de Oceanología

Avenida Ira No. 18406

Cubanacan

La Habana 16

Director: Rodolfo Claso Madruga

$\mathrm{H}$
Instituto Cubano de Hidrografía

$4 \# 302$

Miramar

Director: Angel Joel Chaveco Hernandez

Calle 11, No. 514 


\section{CYPRUS}

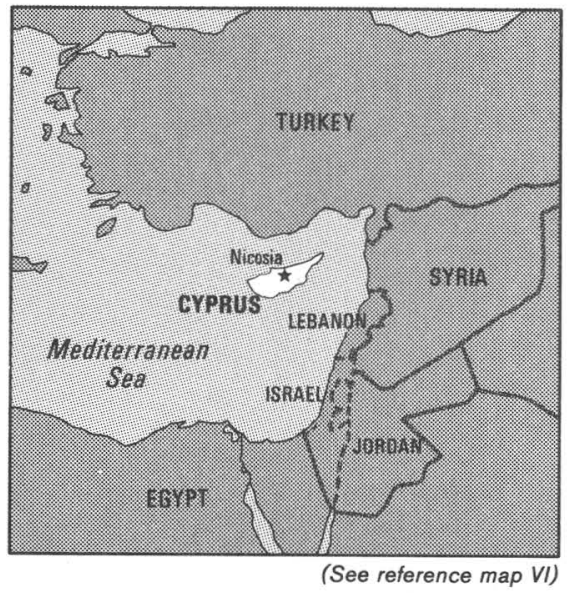

G

C
Geological Survey Department

Ministry of Agriculture and Natural Resources Nicosia

Director: George Constantinou

Department of Lands and Surveys

Ministry of the Interior

Nicosia

Senior Lands and Surveys Officer: Rois G. Nicolaides

Water Development Department

Ministry of Agriculture and Natural Resources

Nicosia

Director: Constantinos Lytras

Department of Mines

Ministry of Commerce and Industry

Nicosia

Senior Mines Officer: Glafkos C. Kronides

\section{CZECHOSLOVAKIA}

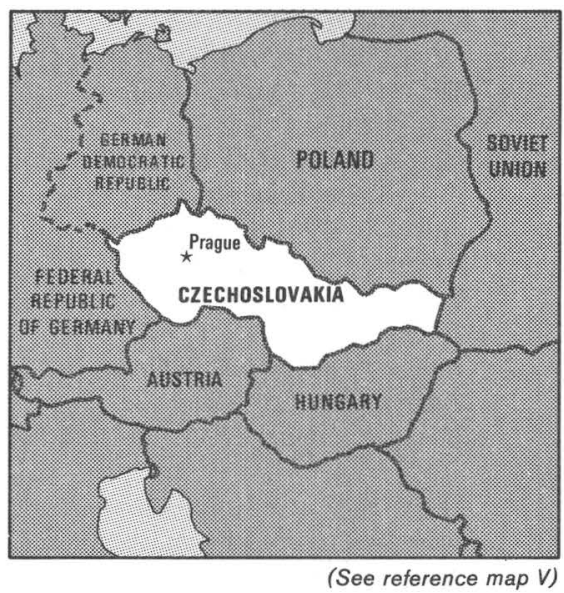

Geografický ustav Č SAV [Geographical Institute of Czechoslovak Academy of Sciences]

Mendelovo nam. 1

66282 Brno

Director: Ing. Vladimir Vahala, Dr. Sc.

Institute of Geology and Geotechnics of the Czechoslovak Academy of Sciences V Holešovičkach 41-

18209 Praha 8 - Liben

Director: Ing. Dr. Gustav Sebor, Dr. Sc.

Geodetický ustav (Geodetic Institute)

Kostelní 42

17030 Praha 7 - Holešovice

Director: Dr. Dimitrij Gebauer

Vyzkumný ustav geodetický, topografický a kartografický

25066 Zdiby 98

Praha - Vychod

Director: Ing. Jaroslav

Kouba

Ustředni ustav geologický

Malostranské namèsti 19

11821 Praha 1 - Mala Strana

Director: Dr. Jaroslav Vacek

Český Hydrometeorologický ustav

[Surface and Ground Water Resources]-Czech Republic

Holečkova 8

15129 Praha 5 - Smichov

Director: Ing. Vaclav Richter

Slovenský Hydrometeorologický ustav

[Surface and Ground Water Resources]-Slovak Republic

Jeseniová 17

88539 Bratislava - Koliba

Director: Dr. Ferdinand Samaj, Dr. Ved 
[Geodetic and Cartographic Administration]

Arbesovo nam. 4

Praha 5 - Smichov

Director: Ing. Jaromir Karnold

$\mathrm{C}$

Vojensky zemepisny ustav

[Military Geographical Institute]

Rooseveltova 23

15001 Praha 6

\section{DENMARK}

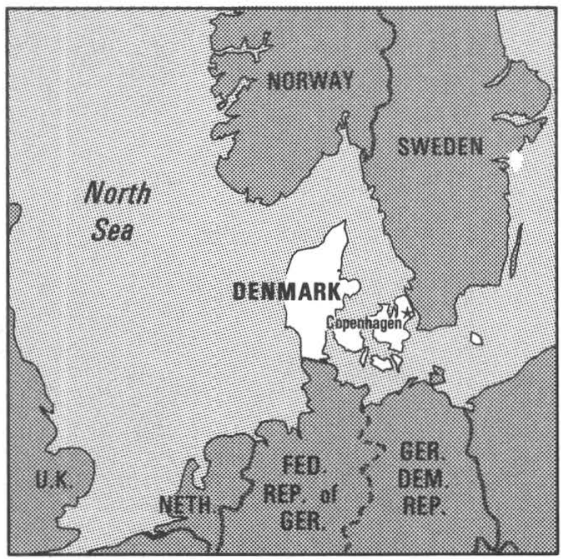

(See reference map $V$ )

G, R Danish Energy Agency

Landemarket 11

DK-1119 Copenhagen K

Director: Hans von Bulow

G Geological Survey of Denmark

Thoravej 31

DK-2400 Copenhagen NV

Director: Ole Berthelsen

G,C,H Ministry for Greenland

Oils and Minerals Department

Hausergade 3

DK-1128 Copenhagen K

Chief of Department: Gert Vigh

H

Hydrological Survey

Nytorv 13

4200 Slagelse

WMO/CHY Representative: Jørgen Lundager Jensen

C Geodetic Institute

Rigsdagsgaarden 7

DK-1218 Copenhagen $\mathrm{K}$

Director: Flemming Wiinblad
Ministry of Industry

Slotholmsgade 12

DK-1216 Copenhagen K

Chief, Office of Energy Legislation and

Concessions: Hagen Jørgensen

\section{DJIBOUTI, REPUBLIC OF}

(No recent information)

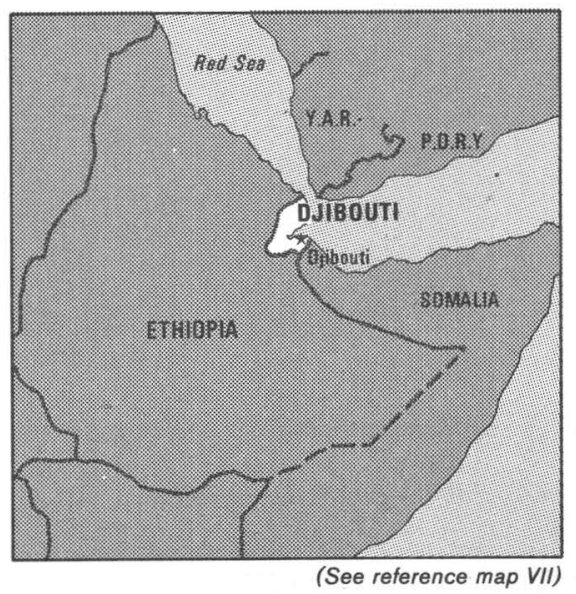

G

Ministère des Travaux Publiques

P.B. 24

Djibouti

Minister: Omar Kamil Warsama

$\mathrm{H}$

Water Authority, Genie Rural

Ministry of Agriculture

Djibouti

Hydrologist: Mohamed Waberi Askar 


\section{DOMINICA}

(No recent information)

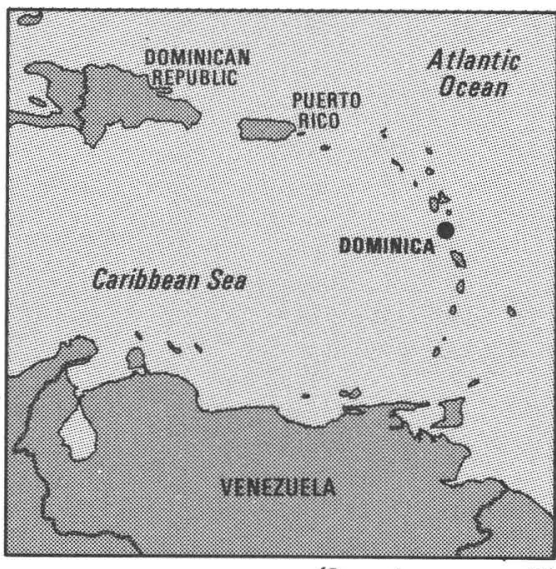

(See reference map III)

\section{G, C,H, R Department of Trade and Industry Roseau \\ Permanent Secretary: Arlington Riviere}

\section{DOMINICAN REPUBLIC}

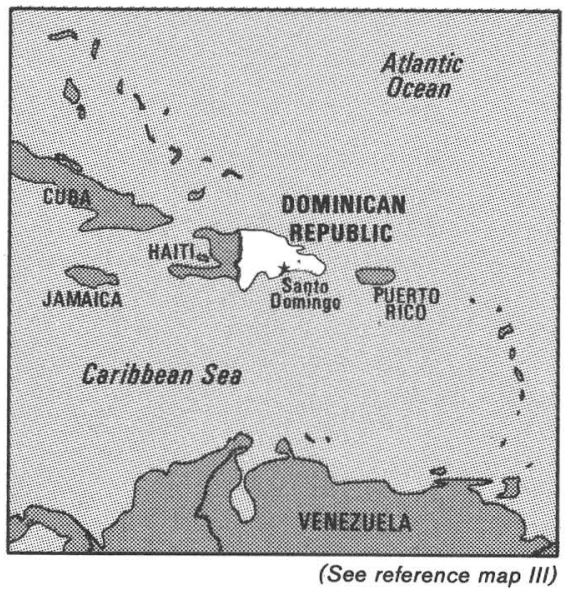

G

Dirección General de Minería

Secretaría de Estado de Industria y Comercio, Ave. Mexico, 11-A, El Huacal

Santo Domingo

Director General: Ing. Miguel A. Peña

G Departamento de Ciencias Geográficas

Facultad de Ciencias

Universidad Autónoma de Santo Domingo

Santo Domingo

H
C

Instituto Geográfico Universitario

Facultad de Ciencias

Universidad Autónoma de Santo Domingo

Santo Domingo

Director: Pablo Smeter

\section{ECUADOR}

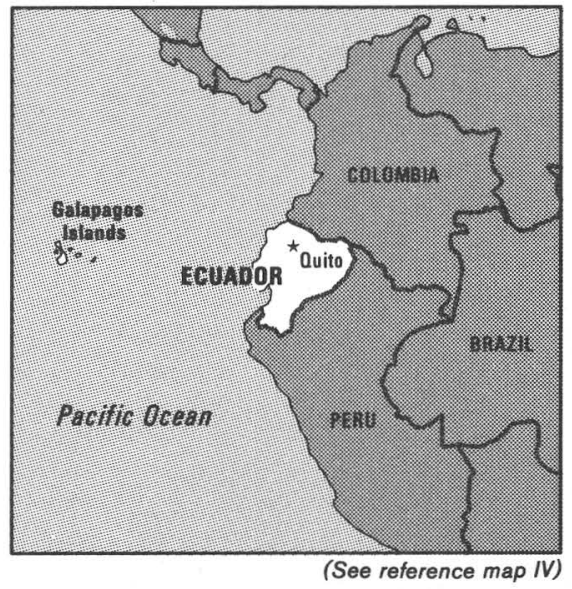

G

G

Dirección General de Geología y Minas Ministerio de Recursos Naturales y Energéticos Carrión \#1016 y Páez

Quito

Director-General: Ing. Pedro Freile Paz y Miño

Centro de Levantamientos Integrados de Recursos Naturales por Sensores Remotos (CLIRSEN) [Center of Integrated Surveys of Natural Resources by Remote Sensing]

Edificio Instituto Geográfico Militar

P.O. Box 8216

Quito

Director Ejecutivo: Ing. Alberto Segovia

Instituto Oceanográfico de la Armada (INOCAR) Casilla 5940

Guayaquil

Director: Fernando Alfaro Echeverria

Instituto Geofísico

Escuela Politécnica National

Apartado 2759

Quito

Director Técnico Encargado: Dr. Minard C. Hall

C Instituto Geográfico Militar (IGM)

Apartado 2435

Quito

Director:. Crnel. de E. M. Ing. Germán Ruiz Zurita 
Departamento de Hidrología

Instituto Nacional de Meteorología e

Hidrología (INAMHI)

Calle Daniel Hidalgo 132 y 10 de Agosto

Quito

Director: Ing. Galo Cisneros Freire

R

Direccion General de Minas e Hidrocarburos

Ministerio de Recursos Naturales y Energéticos

Santa Prisca \#215

Quito

Director General: Ing. Rodrigo Cerón Chamorro

\section{EGYPT}

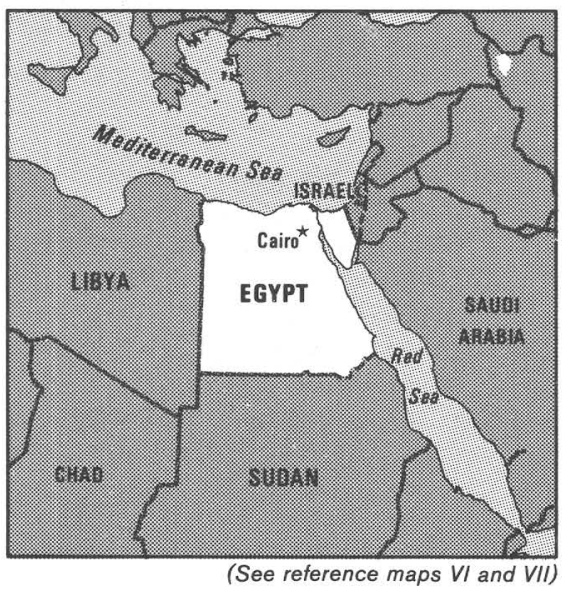

G

Helwan Observatory

c/o Academy of Scientific Research and

Technology

101 Kasr El Aini Street

Cairo

Director: Mohamed Fahim

G

Geological Survey and Mining Authority

4 Saleh Salem Road

Abbasia

Cairo

Acting Chairman: Mahmoud Fawzy El Ramly

G,H Academy of Scientific Research and Technology

101 Kasr El Aini Street

Cairo

President: Dr. Ibrahim Badran

C

Aerial Survey of Egypt

308 El-Haram Street

Giza, Cairo

C

Survey of Egypt
C, G

Egyptian Remote Sensing Center

101 Kasr El Aini Street

Cairo

Chairman: Mohamed Abdel Hady

H Hydrological Research Station

Kanater-al-Khaiyria Barrages

Cairo

Director: A. A. El-Darwish

R

Egyptian General Petroleum Company

Tayaran Street

Nasr City

Cairo

Chairman: Abdel Hady Kandil

G,R Nuclear Materials Authority

P.O. Box 530

Maadi

Cairo

Chairman: Dr. Ibrahim Shazli al-Shazli

\section{EL SALVADOR}

(No recent information)

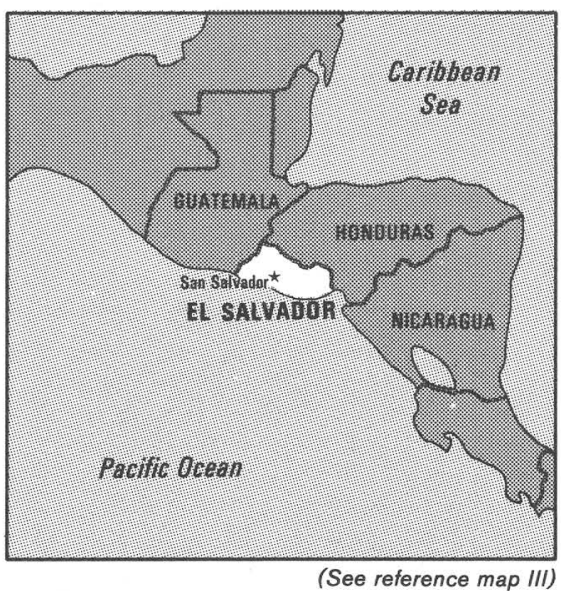

G

Centro de Investigaciones Geotécnicas

Ministerio de Obras Públicas

Avenida Peralta, final, costado Oriente Talleres El Coro

San Salvador

Director: Julio Bran Valencia

$\mathbf{R}$

Comision Nacional del Petróleo

Ministerio de Economía

3a. Calle Poniente 1225

San Salvador

C

Instituto Geográfico Nacional

Sharia El Misaha

Giza

Cairo

Chairman: Dr. Abdel Fattah Habib "Ingeniero Pablo Arnoldo Guzman"

Ministerio de Obras Publicas

Avenida Juan Bertis No. 79

San Salvador

Director: Jose Alberto Gonzalez Garcia 


\section{B,C \\ Ministerio de Obras Publicas \\ Palacio Nacional \\ San Salvador \\ Ministro: Leon Rivas Duran \\ H,C Servicio Hidrológico \\ Direccion General de Riego y Drenaje \\ Ministerio de Agricultura y Ganadería \\ Nueva San Salvador \\ Director General: José Fuente Alas \\ H Servicio Hidrológico \\ Direccion General de Recursos Naturales Renovables \\ Canton El Matasano \\ Soyapango, El Salvador, C.A. \\ Director General: Gonzalo Ernesto Martinez Menéndez \\ WMO/CHy Representative: Hernan Romero Chavarria}

H Servicio Meteorológico Nacional

Ministerio de Agricultura y Ganandería

Nueva San Salvador

Jefe: Gelio Tomás Guzman Lopez

H,C Ministerio de Agricultura y Ganadería

Boulevard Los Héroes y 21 Calle Poniente

San Salvador

Minister: José Rutilio Aquilera

\section{ETHIOPIA}

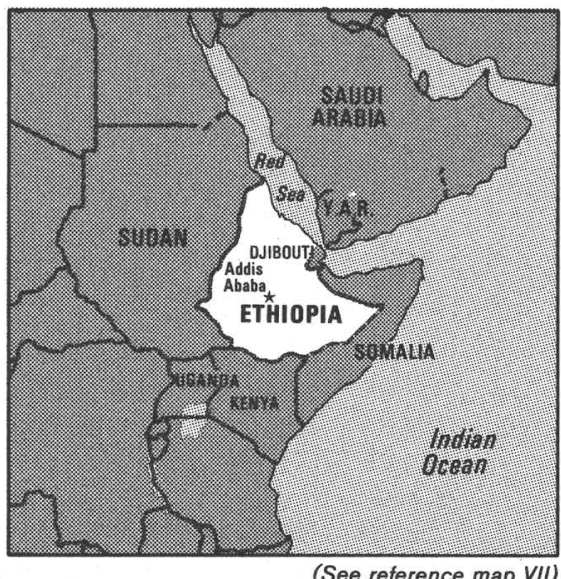

\section{FIJI}

G

Ethiopian Institute of Geological Survey (EIGS)

Ministry of Mines, and Energy

P.O. Box 486

Addis Ababa

General Manager: Ato Getahun Demissie

G, R,H Ministry of Mines, and Energy

P.O. Box 486

Addis Ababa

Minister: Tekeze-Shewa Aytenfisu
C,G,H,R Natural Resources Division

UNECA

Africa Hall

P.O. Box 3001

Addis Ababa

Chief: P. N. Mwanza

C

Cartography Unit

UNECA

Africa Hall

P.O. Box 3001

Addis Ababa

Chief: Funso Olujohungbe

C

Ethiopian Mapping Agency

P.O. Box 597

Addis Ababa

General Manager: Ato Asfaw Fanta

H National Water Resources Commission P.O. Box 1045

Addis Ababa

Commissioner: Ato Alem Alazar

$\mathbf{R}$

Mineral Resources Unit

UNECA

Africa Hall

P.O. Box 3001

Addis Ababa

Officer-in-Charge: F. S. Moshi

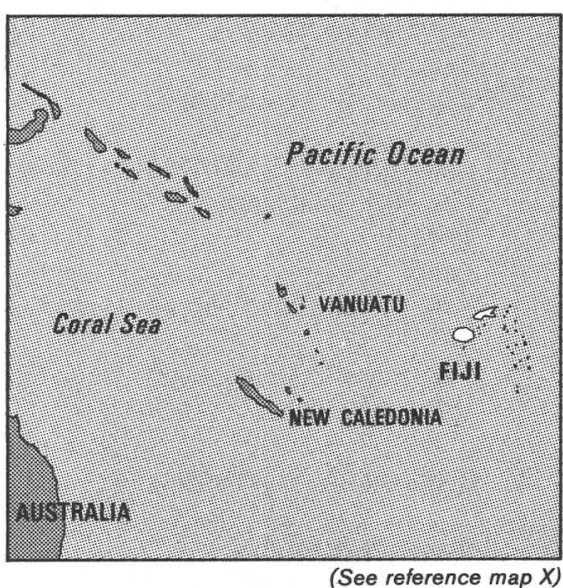

G,R,C Mineral Resources Division

Ministry of Energy and Mineral Resources Private Mail Bag, G.P.O.

Suva

Acting Director for Mineral Development:

A. Rahiman 
Department of Lands and Survey

Ministry of Lands, Local Government and Housing

P.O. Box 2222, Government Building

Suva

Director:. Brij Lal

$\mathrm{H}$

Hydraulics Section

Public Works Department

Ministry of Communications and Works

Ganilau House

Suva

Director: R.H. Sheratt

\section{FINLAND}

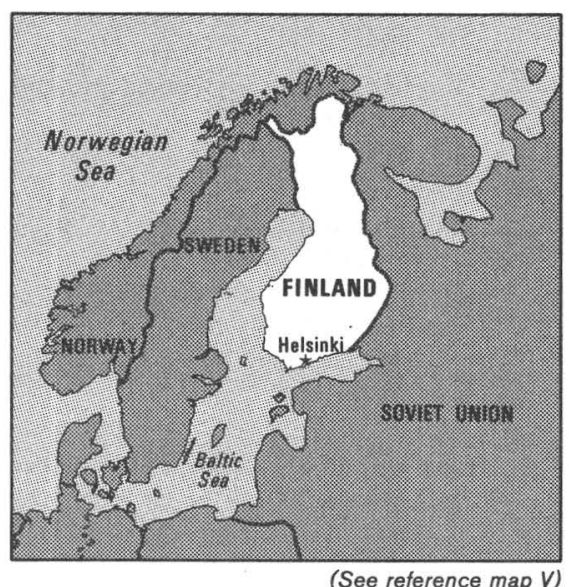

G

Geologinen Tutkimuskeskus

[Geological Survey of Finland]

Kivimiehentie 1

02150 Espoo 15

Director: Prof. L. K. Kauranne

G

Geotekniikan Laboratorio

[Geotechnical Laboratory under the State Technical Research Center]

Lämpömiehenkuja 2 B

02150 Espoo 15

Director:. Prof. Markku Tammirinne

C

Maanmittaushallitus

[National Board of Survey]

PL 84

00521 Helsinki 52

General Director: Lauri Kantee

C

Maanmittaushallituksen Karttapaino

[National Board of Survey, Publication

Division]

PL 85

00521 Helsinki 52

Office Chief: Osmo Niemelä
C

Geodeettinen Laitos

[Geodetic Institute]

Ilmalankatu $1 \mathrm{~A}$

00240 Helsinki 24

Director:. Prof. Juhani Kakkuri

$\mathrm{H}$

Vesitutkimustoimisto

PL 250

[Water Research Office]

0010 Helsinki 10

Office Chief: Reino Laaksonen

$\mathrm{H}$
Hydrologian Toimisto
PL 436
[Hydrological Office]
00101 Helsinki 10
Office Chief: Risto Lemmelä

\section{FRANCE}

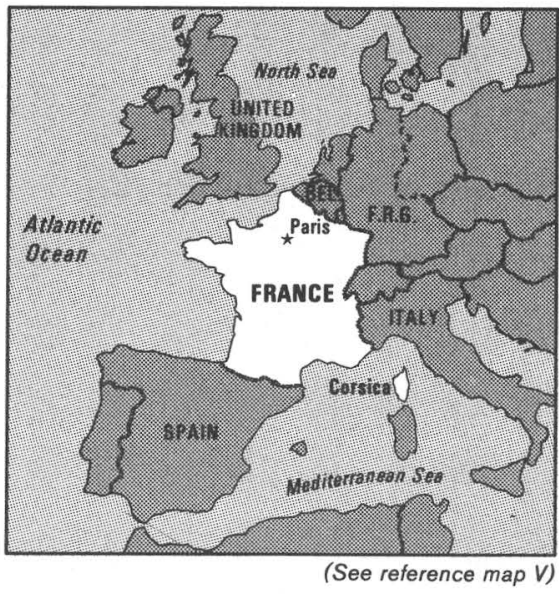

G

Centre National de la Recherche Scientifique 15 , quai Anatole-France 75700 Paris

President: Claude Frejacques

Directeur-General: Pierre Papon

G,H, R Bureau de Recherches Géologiques et Minières (BRGM)

B.P. 6009

45060 Orléans Cedex

Director: Hubert de la Roche

G

Institut Francais de Pétrole

4 , avenue de Bois-Preau

92502 Reuil Malmaison

Directeur-General: Jean-Claude Balaceanu

G

Office de la Recherche Scientifique et Technique Outre-Mer (ORSTOM)

[Office of Scientific and Technical Research Overseas]

24, rue Bayard

75008 Paris

Directeur General: Alain Ruellan 

Risques Naturels Majeurs

25 , avenue Charles-Floquet

75700 Paris

Commissaire: Haroun Tazieff

H

Electricité de France

134, boulevard Haussman

75336 Paris Cedex 8

C

Laboratoire d'Information et de Documentation en Géographie

191, rue St. Jacques

75114 Paris

Director: Michel Drain

C

Centre d'Etude et de Réalisations Cartographiques et Géographiques

191 , rue St. Jacques

75114 Paris

Director: Fernand Joly

C

Institut Géographique National

136 bis, rue de Grenelle

75700 Paris

Director: Jean Winghart

\section{FRENCH GUIANA}

(No recent information)

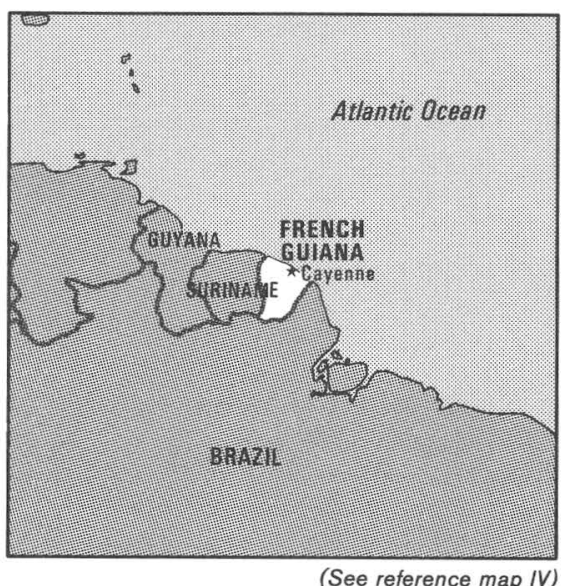

G

Bureau des Recherches Géologiques et Minières (BRGM)

B.P. 42

Cayenne

\section{GABON}

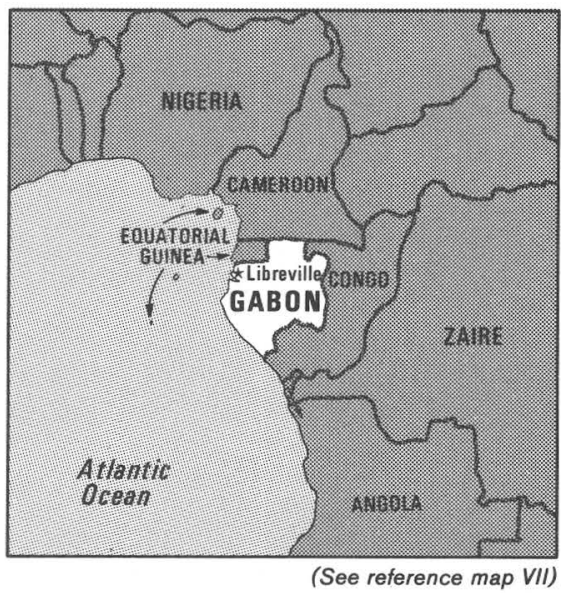

G,C

$\mathbf{R}$

\section{GAMBIA, THE}

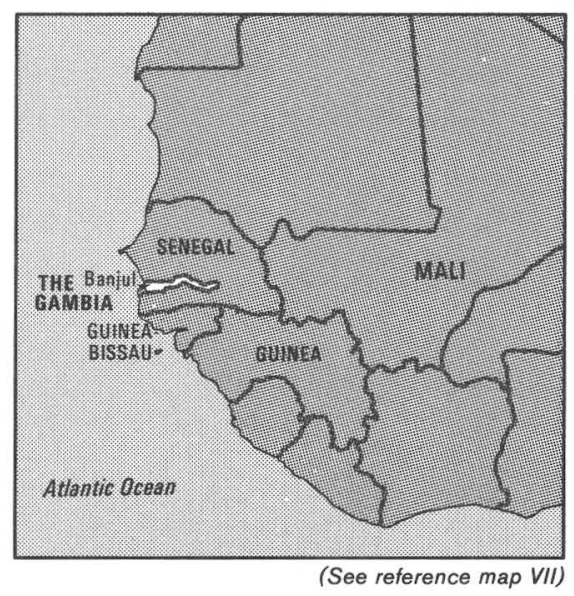

C

Survey Department

Half Die, Banjul

Director of Surveys: Mactarr O. Jobe 
Department of Hydrometeorological Services

7, Marina Parade

Banjul

Director: Edi N'jie

Ministry of Water Resources and the Environment 6, Marina Parade

Banjul

Permanent Secretary: O. N. R. Othman

H

L'Organization pour la Mise en Valeur du Fleuve Gambie (OMVG)

[Organization for the Development of the Gambia River Basin]

Chairman: Malick John

H Department of Hydrometeorological Services

7 Marina Parade

Banjul

Director: Edi N'jie
Kaolack, Senegal

\section{GERMAN DEMOCRATIC REPUBLIC}

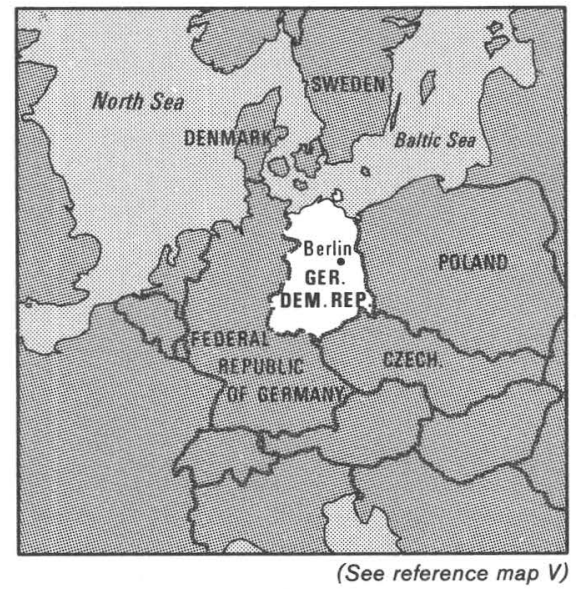

G

Zentrale Vorratskommission für Mineralogische Rohstoffe

Invalidenstrasse 44

1040 Berlin

G

Zentrales Geologisches Institut

Invalidenstrasse 44

1040 Berlin

Director: Dr. Karlheinz Bendsko

Gesellschaft für Geologische Wissenschaften der DDR

Invalidenstrasse 43

1040 Berlin
Akademie der Wissenschaften

Zentralinstitut für die Physik der Erde 1500 Potsdam Telegrafenberg

Director: Prof. Dr. Heinz Kautzleben

Institut für Bodenkunde

Abt. Standortkartierung

Clara-Zetkin-Strasse 105

1080 Berlin

Deutsche Buecherei

Abteilung Beschaffung

Dienststelle für Schriften des Auslandes

Deutscher Platz 1

7010 Leipzig

Institut für Wasserwirtschaft

Schnellerstrasse 140

1190 Berlin

Director: Dr. Peter Loese

Kammer der Technik

Fachverband Wasser

Clara-Zetkin-Strasse 115/117

1080 Berlin

\section{GERMANY, FEDERAL REPUBLIC OF}

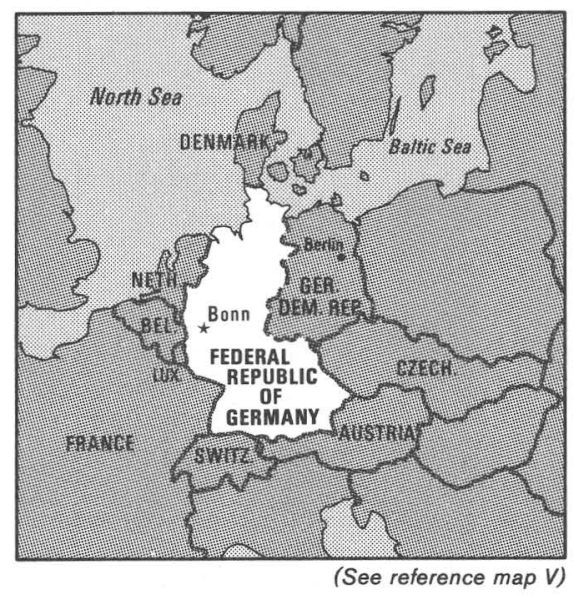

G,C,H Bundesminister der Verteidigung

Streitkraefteamt

Militaerisches Geowesen

Theaterplatz 10-18

5300 Bonn 2

President: Jürgen H. Denker

G,R,C Bundesanstalt für Geowissenschaften und Rohstoffe (BGR) (Geobund)

Alfred-Bentz-Haus

Postfach 510153

Stilleweg 2

3000 Hannover 51

President: Friedrich N. Bender 
$\mathrm{H}, \mathrm{R}, \mathrm{C}, \mathrm{G}$

Deutsches Hydrographisches Institut (DHI)

Bernhard-Nocht-Strasse 78

2000 Hamburg 4

President: Gerhard Zickwolff

$\mathrm{H}$

Bundesanstalt für Gewasserkunde

Kaiserin-Augusta-Andagen 15-17

5400 Koblenz

President: Herbert Knoepp

Arbeitsgemeinshaft der Vermessungsverwaltungen der Laender der Bundesrepublik Deutschland (AdV)

Warmbuechenkamp 2

3000 Hannover 1

Vorsitzer: Dr. Ing. Harald Lucht

Geschäfts fuhrer: Ministerialrat Wolf-Erich Von Daack

Institut für Angewandte Geodaesie

Richard-Strauss-Allee 11

6000 Frankfurt am Main 70

Director: Walter Satzinger

\section{GHANA}

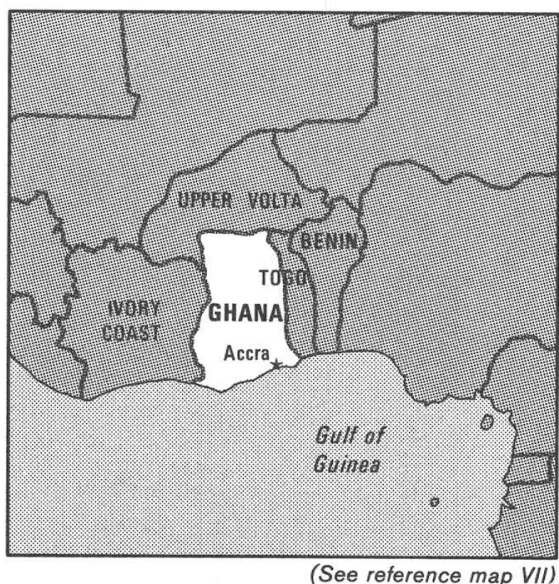

G

Industrial Research Institute

Council for Scientific and Industrial Research (CSIR)

P.O. Box M-32

Accra

Acting Director: Alexandria Amoako-Mensah

G

Geological Survey of Ghana

P.O. Box M-80

Accra

Director: G. O. Kesse

$\mathrm{H}$

Water Resources Research Unit

Council for Scientific and Industrial Research

P.O. Box M-32

Accra

Acting Director: Nii Boi Ayibotele
Hydrological Branch

Public Works Department

P.O. Box 136

Accra

Chief Hydrological Engineer: S. A. Acheampong

C

Ghana Geographical Association

University of Ghana

Legon

President: Prof. George Benneh

C

Survey Division

P.O. Box 191

Cantonments

Accra

Acting Chief Survey Officer: I. Abu

C

Buildings and Roads Research Institute

Council of Scientific and Industrial Research (CSIR)

P.O. Box 40

University Post Office

Kumasi

Acting Director: M. D. Gidigasu

R

$\mathbf{R}$

Ministry of Lands and Natural Resources

P. O. Box M-212

Accra

Principal Secretary: W. K. Alomatu

Ministry of Fuel and Power

P.O. Box M-212

Accra

Principal Secretary: J. K. Owusu

\section{GREECE}

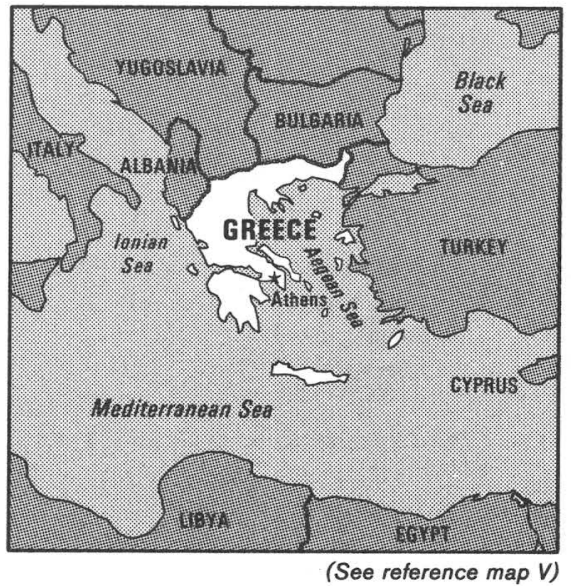

G

Institute of Geological and Mining Research (IGME)

70 Mesogion Street

GR-115 27 Athens 
54 Acadimias Street

GR-106 79 Athens

H

National Meteorological Service (EMY)

Climate, Agriculture and Hydrology Division

Athens Airport-East

Hellenikon Postal Service

GR-166 03 Athens

Geological and Hydrological Directorate

Ministry of Agriculture

46 Halkokondyli Street

GR-104 38 Athens

Hydraulic Works Engineering Division (BM3)

Ministry of Public Works

15 Panagi Tsaldari Street

Kalithea

GR-176 76 Athens

Hydraulic Works Construction and Maintenance Service (BY)

Ministry of Public Works

15 Panagi Tsaldari Street

Kalithea

GR-176 76 Athens

C Hellenic Army Geographic Service

3 Evelpidon Street

GR-113 62 Athens

R

4th Directorate of Mines

Ministry of Energy and Natural Resources

80 Michalakopoulou Street

GR-115 28 Athens

$\mathbf{R}$

National Petroleum Products Service Ministry of Energy and Natural Resources 80 Michalakopoulou Street

GR-115 28 Athens

\section{GREENLAND}

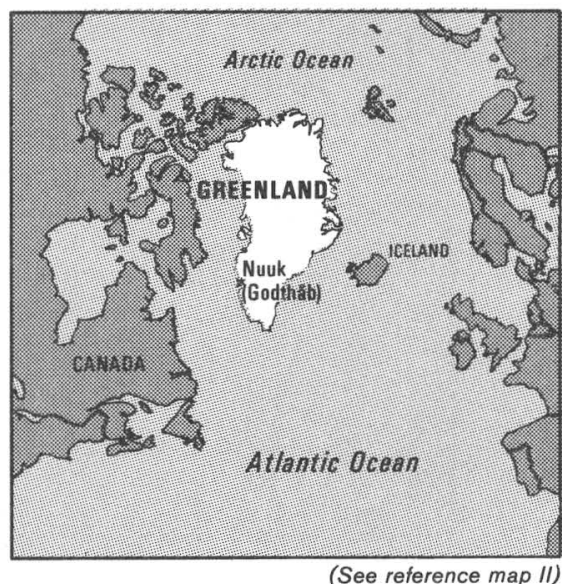

G

Grønlands Geologiske Unders $\varnothing$ gelser

Ostervoldgade 10, Tr. KL

DK-1350 Copenhagen K

Denmark

Director: Dr. Martin Ghisler

\section{GUADELOUPE}

(No recent information)

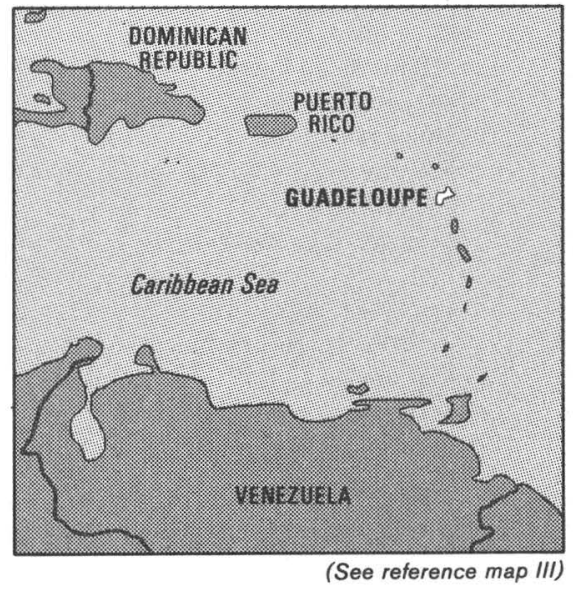

G

Arrondissement Minéralogique de la Guyane B.P. 448

Pointe-a-Pitre

H

Office de la Recherche Scientifique et Technique Outre-Mer (ORSTOM) 6

B.P. 504

97165 Pointe-a-Pitre

Cedex

F 1960

\footnotetext{
${ }^{6}$ French research organization; no governmental affiliation.
} 


\section{GUATEMALA}

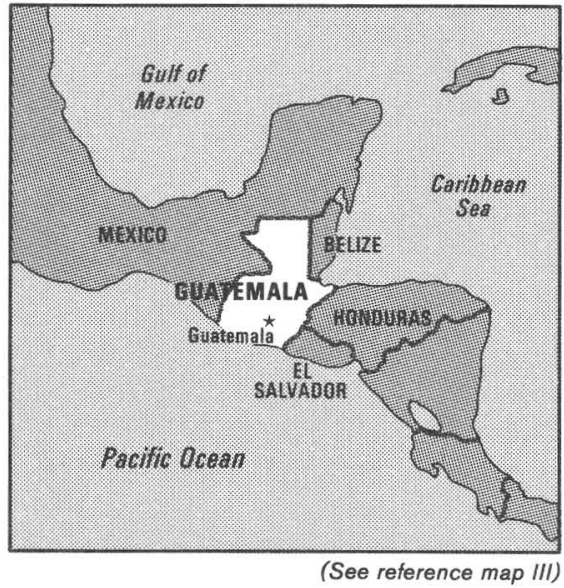

G,H Instituto de Sismología, Vulcanología,

Meteorología, e Hidrología (INSIVUMEH)

7 Avenida 14-57, Zona 13

Guatemala, C.A.

Director: Ing. Estuardo Velasquez Vásquez

G

Division de Geología

Instituto Geográfico Militar

Avenida Las Américas 5-76, Zona 13

Guatemala, C.A.

Director: Ing. Oscar Salazar Contreras

G,R Ministerio de Energía y Minería

Diagonal 17, 29-78, Zona 11

Guatemala, C.A.

Secretario General: Ing. Alejandro Contreras Bonilla

Minister: Ing. Alejandro Sigfrido Contreras

C Instituto Geográfico Militar

Avenida de las Americas 5-76, Zona 13

Guatemala, C.A.

Director: Coronel de Ingenieros Ing. Lionel Anibal Rivera Morataya

\section{GUINEA}

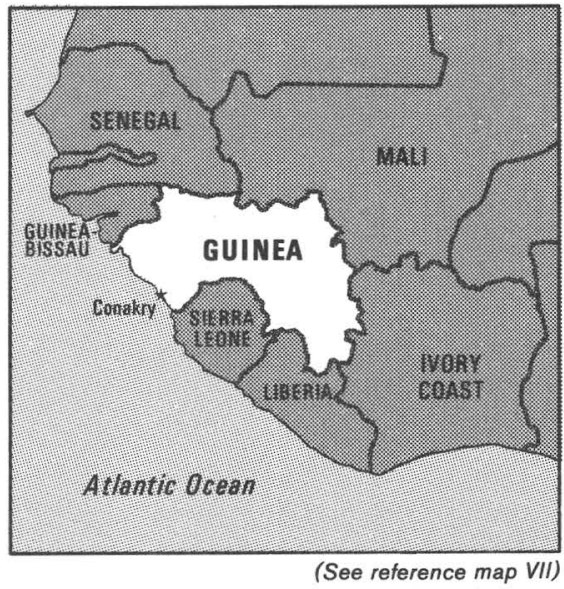

\section{G,C,R Ministry of Mines and Geológy \\ B.P. 295 \\ Conakry \\ Minister: Captain Jean Traore}

$\mathrm{H}$

Ministry of Agriculture

B.P. 576

Conakry

Minister: Chief of Battalion Alseny Fofana

Director General of Waters and Forests:

Khalidou Diallo

\section{GUINEA-BISSAU}

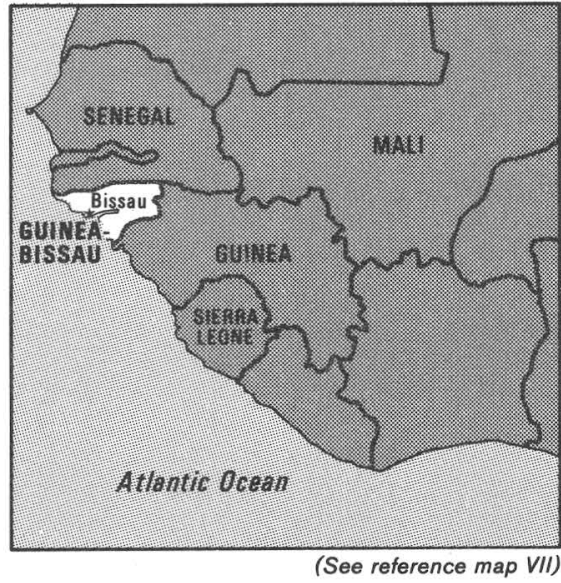

C, G,H, R Ministry of Natural Resources

Caixa Postal 399

Bissau

Minister: Joseph Turpin

Director General: Mario Ferreira Silva 


\section{GUYANA}

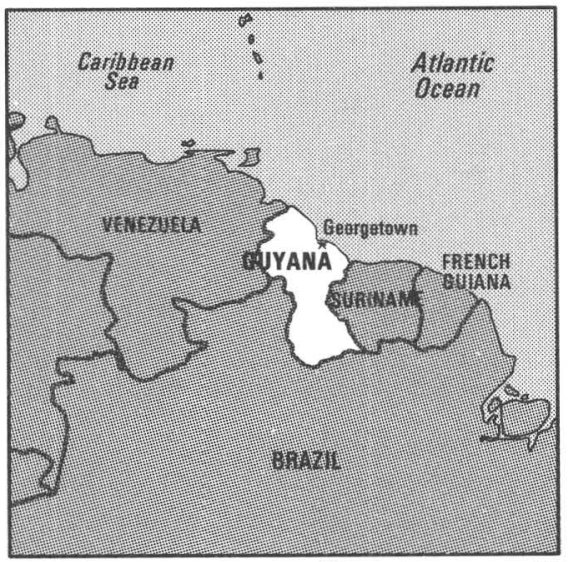

(See reference map IV)

Geological Surveys and Mines Department

P.O. Box 1028

Brickdam, Georgetown

Commissioner of Geology and Mines:

Grantley Walrond

H Hydrometeorological Service

Ministry of Works and Transport

P.O. Box 26

Georgetown

Chief: K. E. D. Potter

C

Lands and Surveys Department

Ministry of Agriculture

Upper Hadfield Street

Georgetown

Commissioner of Lands and Surveys: T. A. Earle

R

Petroleum Unit

\section{HAITI}

(No recent information)

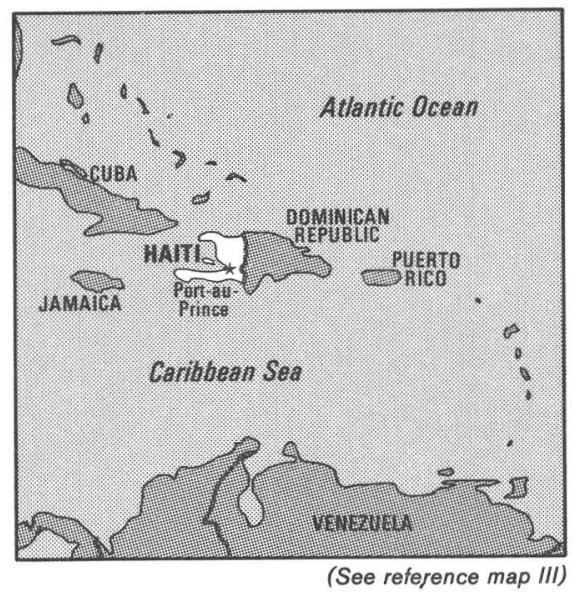

G,R Ministry of Mines and Energy Resources Village Willy Lamothe, Delmas 9

P.O. Box 2174

Port-au-Prince

Secretary of State: Claude Mompoint

Director General: Michel Simon

$\mathrm{H}$

Service Métérologique et Hydrologique

Département de l'Agriculture, des Ressources Naturelles et du Développement Rural

Damier, Port-au-Prince

Director: Alexandre Gontier

C

Service de Géodésie et de Cartographie

Boulevard Harry Truman

Cité de l'Exposition

Port-au-Prince

Director: Raymond B. Oriol

Ministry of Energy and Mines

Brickdam and Boyle Place

Georgetown

Chief: B. Sucre 
HONDURAS

(No recent information)

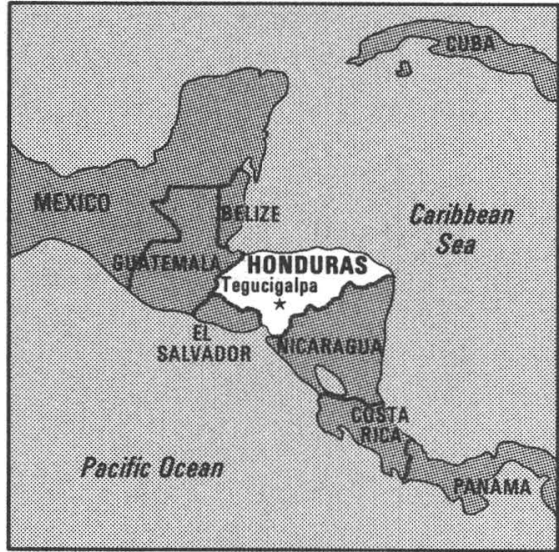

(See reference map III)

G

Exploracion Geotérmica

Empresa Nacional de Energía Eléctrica (ENEE)

Apartado 99

Tegucigalpa, D.C.

Chief: Werner Hugo Falck C.

G,C,R Dirección General de Minas e Hidrocarbouros

Ministerio de Recursos Naturales

Boulevard Centro América

Tegucigalpa, D.C.

Director-General: Edgardo Zuñiga R.

$\mathrm{H}$

$\mathrm{H}$

C

Servicio Autónomo Nacional de Acueductor y

Alcantarillados (SANAA)

Apartado Postal 437

Tegucigalpa, D.C.

Director-General: Rubén Flores Guillén

Servicios Hidrológicos y Climatológicos

Ministerio de Recursos Naturales

Apartado 1839

Tegucigalpa, D.C.

Chief: M. Lardizábal Becerra

Instituto Geográfico Nacional (IGN)

Apartado Postal 758

Barrio La Balsa

Comayaguela D.C.

Director-General: José Edmundo Alcerro P.

C Oficina Nacional de Programa Catastral

Edificio Didemo, 4 Piso

Tegucigalpa, D.C.

Director: Julio Cesar Zepeda

R

Comisión Nacional de Energía

Ministerio de Economía y Comercio

Altos Edif. Banco de Londres y Montreal

Tegucigalpa, D.C.

Director: J. Dalmiro Caballero

\section{HONG KONG}

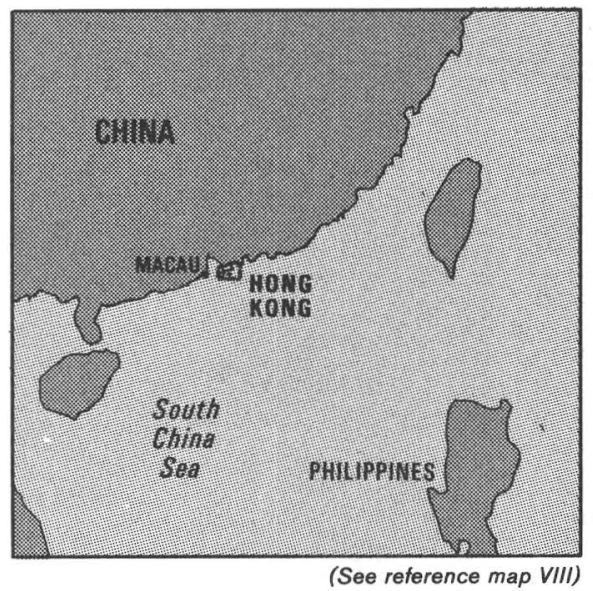

G

Mines Division

Canton Road Government Offices

Canton Road

Kowloon

Superintendent of Mines:. Borhylen Wang

G

Department of Geography and Geology

University of Hong Kong

$\mathrm{H}$

Royal Observatory

134A, Nathan Road

Kowloon

\section{HUNGARY}

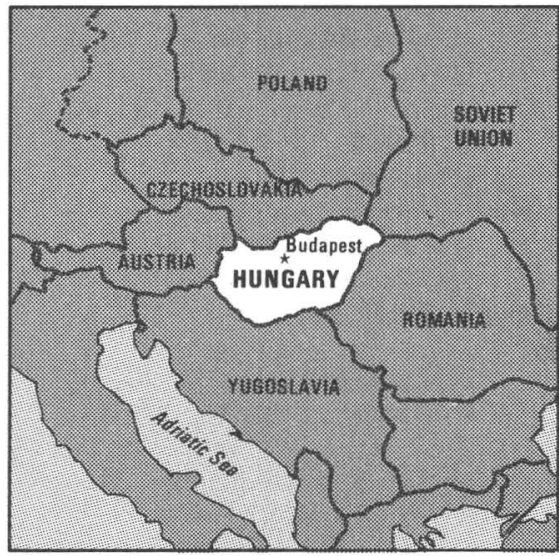

(See reference map V)

G

Központi Földtani Hivatal

[Central Office of Geology]

H-1011 Budapest, I.

Iskola u. 19-27

Chairman: Dr. József Fülöp 
Magyar Állami Földtani Intézet [Hungarian Geological Institute]

H-1143 Budapest, XIV.

Népstadion út 14

Director: Dr. Géza Hámor

Országos Koölajés Gásipari Tröszt

Geofizikai Kutatasi Vallalat

[Geophysical Exploration Co.]

H-1068 Budapest, VI.

Gorkij Fasor 42

Director: Karoly Molnar

Országos Koölajés Gázipari Tröszt

Magyar Szenhidrogenipari Kutató-Fejleszto

Intézet

[Hungarian Hydrocarbon Institute]

H-2443 Szazhalombatta

P.O. Box 32

Director: Dr. Sandor Doleschall

Magyar Állami Eötvós Lóránd Geofizikai Intézet [Hungarian Geophysical Institute L. Eotvos]

H-1145 Budapest

Columbus u. 17-23

Director: Dr. Pál Müller

Magyar Tudományos Akadémia

[Hungarian Academy of Sciences]

Geokémiai Kutatólaboratóriuma

[Geochemical Research Laboratory]

H-1112 Budapest, XI.

Budaorsi ut 45

Director: Dr. György Pántó

G,C Magyar Tudományos Akadémia

[Hungarian Academy of Science]

Geodéziai es Geofizikai Kutató Intézete

[Research Institute for Geodesy and

Geophysics]

H-9400 Sopron

Múzeum u. 6

Director: Dr. József Somogyi

Országos Koölajés Gazipari Troszt

[National Crude Oil and Natural Gas Trust]

H-1117 Budapest, XI.

Schonherz Z. u. 18

Director General: Dr. Istvan Zsengeller

Magyar Állami Vizügyi Hivatal

[National Water Authority]

P.O. Box 351

H-1394 Budapest

Vizgazdálkodási Tudományos Kutató Intézet (VITUKI)

[Research Institute for Water Resources

Development]

H-1095 Budapest, IX.

Kvassay Jenö u. 1

H-1095

Director: Dr. Karoly Stelczer
Vizgazdálkodási Intézet

[Institute for Water Management]

H-1054 Budapest, V.

Alkotmany u. 29

Director: Dr. Rudolf Muts

C

MEM Országos Földügyi es Terkepeszeti Hivatal

[National Cadaster and Cartography Bureau]

H-1055 Budapest, V.

Kossuth Lajos tér 11

\section{ICELAND}

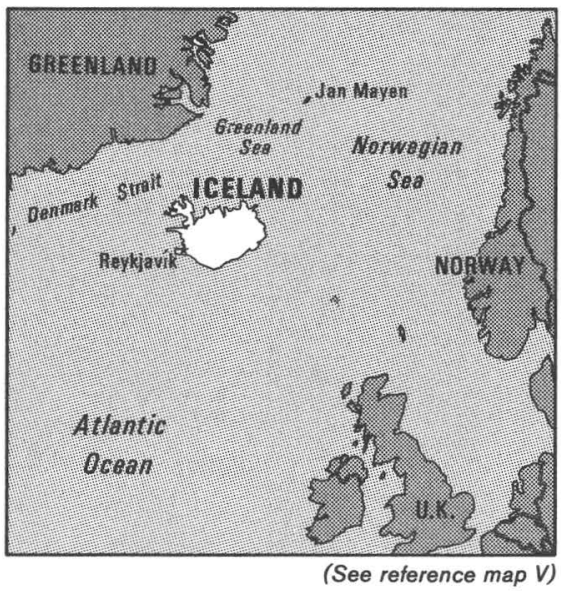

National Energy Authority of Iceland

Grensásvegur 9

108 Reykjavik

Director-General: Jakob Björnsson

Geothermal Division

National Energy Authority

Grensásvegur 9

108 Reykjavik

Director: Dr. Gudmundur Pálmason

Nordic Volcanological Institute

University of Iceland

Geoscience Building

Sudurgata

101 Reykjavik

Director: Dr. Gudmundur E. Sigvaldason

Division of Geology and Geography

Museum of Natural History

Laugavegi 105 and Hverfisgöta 116

P.O. Box 5320

105 Reykjavik,

Director: Dr. Sveinn P. Jakobsson

Marine Research Institute

Skulagötu 4

101 Reykjavik,

Director: Jón Jónsson 
Grensásvegur 9

108 Reykjavik

Director: Dr. Haukur Tómasson

C Iceland Geodetic Survey

Laugavegi 178

105 Reykjavik

Director: Bragi Gudmundsson

G

University of Science Institute

University of Iceland

Dunhagi 3

107 Reykjavik

Director: Dr. Sveinbjörn Björnsson

\section{INDIA}

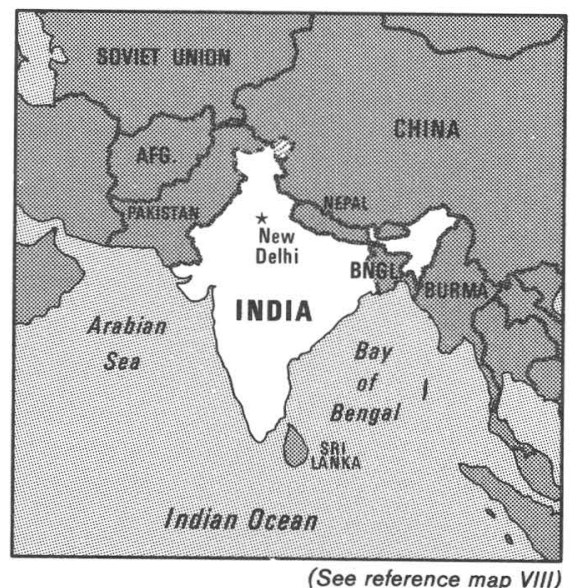

G

G
G,C,H National Remote Sensing Agency

Department of Science and Technology

No. 4 Sardar Patel Road

Secunderabad 500003

Andhra Pradesh

Director: Prof. B. L. Deekshatulu

C

C

C

Central Water Commission
Survey of India

Surveyor General Office

P.O. Box 37

Dehra Dun 248001

Surveyor General of India: K. L. Khosla

Geodetic and Geophysical Surveys

Survey of India

Dehra Dun 248001

Director: M. G. Arur

Indian Photo-Interpretation Institute (NRSA)

4, Kalidas Road

P.B. No. 235

Dehra Dun, Uttar Pradesh

India

Dean: Col. P. Misra (Retd.)

Central Ground Water Board

Ministry of Agriculture and Irrigation

Jam Nagar House

New Delhi 110001

Director: B. P. C. Sinha

Bikaner House, Shahjahan Road

New Delhi 110011

Chairman: M. G. Padhye

Central Board of Irrigation and Power

Kasturba Gandhi Marg

New Delhi 110001

Secretary: C. V. J. Varma

India Meteorological Department

Lodi Road

New Delhi 110003

Director General: P. K. Das

Ministry of Petroleum, Chemicals and Fertilizers

Shastari Bhavan

Dr. Rajendra Prasad Road

New Delhi 110001

Secretary: A. S. Gill 
Department of Science and Technology

Earth Sciences Division

Technology Bhawan off Mehrauli Road

New Delhi 110016

Advisor: M. N. Qureshy

\section{INDONESIA}

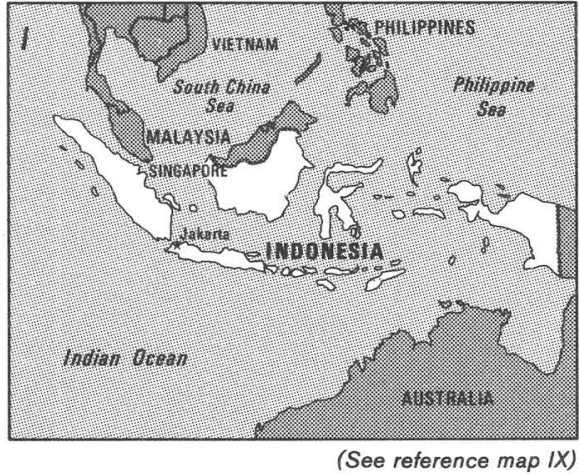

G

$\mathrm{G}, \mathrm{C}, \mathrm{H}$

G, R

G, R
Badan Meteorologi dan Geofisika

[Meteorological and Geophysical Institute]

Ministry of Communication

Jalan Arif Rachman Hakim 3, Jakarta Pusat

Jakarta

Director: C. Sutrisno

Direktorat Sumber Daya Mineral

[Directorate of Mineral Resources]

Jalan Diponegoro 57

Bandung

Director: Salman Padmanagara

Departemen Pertambangan Dan Energi

[Ministry of Mines and Energy]

Jalan Merdeka Selatan 18, Jakarta Pusat

Jakarta

Minister: Prof. Dr. Subroto, H.E.

$\mathrm{H}$

Direktorat Jenderal Pertambangan Umum

[Directorate General of Mining]

Jalan Jenderal Gatot Subrote Kav. 49, Jakarta Pusat

Jakarta

Director General of Mines: Prof. Dr. John A. Katili

Direktorat Jenderal Minyak dan Gas Bumi (MIGAS) [Directorate General of Oil and Gas]

Jalan M. H. Thamrin 1, Jakarta Pusat

Jakarta

Director General: Wijarso

Pusat Pengembangan Teknologi Minyak Dan

Gas Bumi [Oil and Gas Technology

Development Centre]

Jalan Cipulir, Kebayoran Lama, Jakarta Selatan

Jakarta

Director: Prof. Dr. Wahyudi Wisaksono
Lembaga Oceanologi Nasional

[National Institute of Oceanology]

Komplek Bina Samudera

Jalan Pasir Putih 1, Ancol Timur

P.O. Box 580 DAK

Jakarta

Director: A. Sugiarto

Jawatan Topografi Angkatan Darat (JATOP)

[Army Topographic Service]

Jalan Gunung Sahari 90

Jakarta

Director: Brig. General Rahardjo Dirdjosaputro

Direktorat Tata Guna Tanah

[Directorate of Land Use]

Ministry of Internal Affairs

Jalan Sisingamangaraja 2, Jakarta Selatan

Kebayoran, Jakarta

Director: I. M. Sandy

Pusat Survai dan Pemetaan A.B.R.J. (Surta

A.B.R.J.) [Armed Forces Surveys and Mapping Agency]

Jalan Dr. Wahidin I/11, Jakarta Pusat

Jakarta

Chief: First Marshall D. U. Martoyo

Badan Koordinasi Survai Dan Pemetaan

Nasional (Bakosurtanal) [National

Coordinating Body for Survey and Mapping]

Jalan Raya Jakarta km 46

Cibinong, Bogor

Chairman: Pranoto Asmoro

Jawatan Hidro-Oseanografi Angkatan Laut (JANHIDROS) [Navy Hydro-Oceanographic Institute]

Jalan Gunung Sahari 87

Jakarta

Chief: Navy Kolonel P. L. Katoppo

Departemen Pekerjaan Umum

[Ministry of Public Works]

Jalan Pattimura 20

Kebayoran, Jakarta

Minister: Soeyono Sosrodarsono (H.E.)

Jawatan Pemotretan Udara

[Aerial Mapping Service]

Komplek Lapangan Udara Halim Perdanadusuma

Jakarta

Chief: First Marshall Suhardjo

Pusat Penelitian dan Pengembangan Geologi

[Geological Research and Development Center]

Jalan Diponegoro 57

Bandung

Director: Hartono

Pusat Pengembangan Teknologi Mineral

[Mineral Technology Development Centre]

Jalan Jenderal Sudirman 623

Bandung

Director: Bambang Sulasmoro 
Jalan Diponogoro 57

Bandung

Director: A. Sudradjat

Direktorat Geologi Tata Lingkungan [Directorate of Environmental Geology] Jalan Diponogoro 57

Bandung

Director: E. J. Patty

\section{IRAN}

(No recent information)

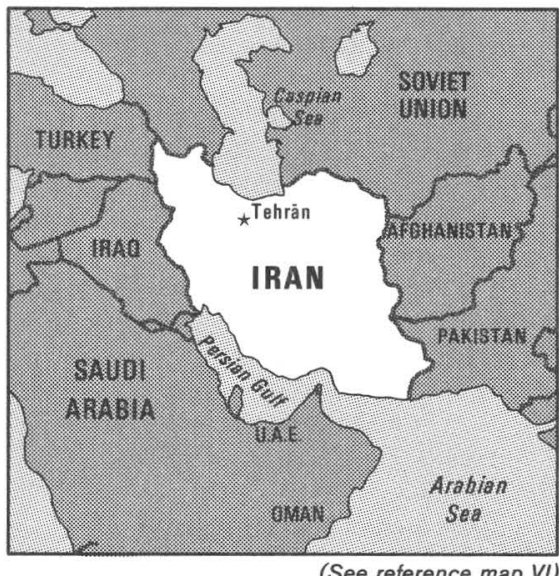

G

G

G

C
H

$\begin{array}{ll} & 64 \text { Ghadessi Street, North } \\ & \text { Boulevard Elizabeth } \\ \text { Tehrān } \\ \text { Director: S. Nazhand }\end{array}$

IRAQ

(No recent information)

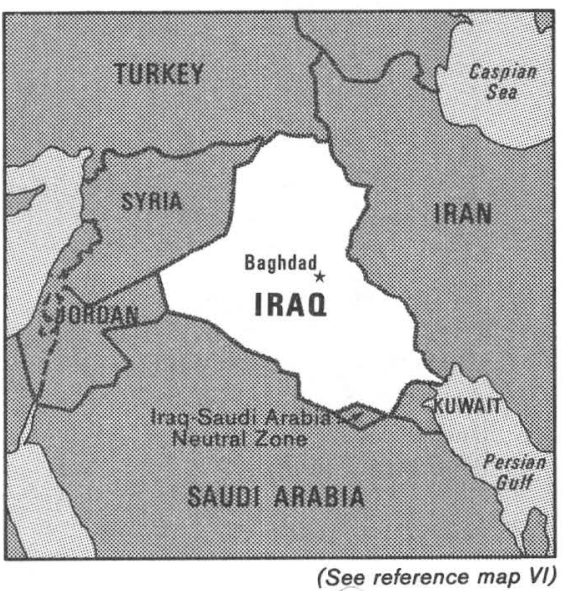

G, R,C Directorate General of Geological Survey and Mineral Investigation

State Organization for Minerals

P.O. Box 986 - Alwiyah

Baghdad

G,R,C State Establishment for Oil and Gas Exploration Iraqi National Oil Company

P.O. Box 476

Khullani Square

Baghdad

H,C State Establishment for Studies and Design State Organization for Dams

P.O. Box 11001 - Karadah

Baghdad

H,C State Organization for Groundwater Investment P.O. Box 3231 - Waziriyah

Baghdad

C

State Establishment for Surveying

Ministry of Irrigation

P.O. Box 5813

Gailani Square

Baghdad 
Scientific Research Council

P.O. Box 2416 - Jadiriyah

Baghdad

Seismology Unit

Building Research Center

Scientific Research Council

P.O. Box 255 - Jadiriyah

Baghdad

G, R Petroleum and Gas Engineering Department

Petroleum Research Center

Scientific Research Council

P.O. Box 10039 - Jadiriyah

Baghdad

\section{IRELAND}

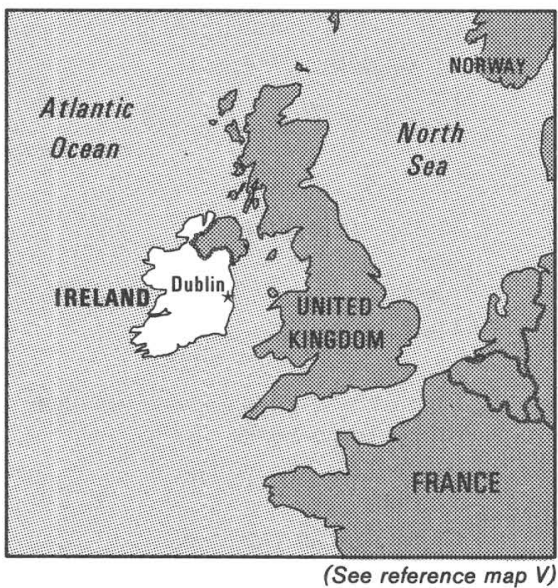

G

G,H Institute for Petroleum Research and Geophysics P.O. Box 1717

Holon

Director: Zvi Benari

C

Department of Surveys

Ministry of Labor

P.O. Box 14171

1 Rehov Lincoln

Tel Aviv

Director: Ron Edward

Hydrological Service

Geological Survey of Ireland

Ministry of Agriculture, Water Commission

14 Hume Street

Dublin 2

Director: Dr. C. E. F. Williams

Yirmiyahu Street, 50

Jerusalem 91060

Director: Martin Jacobs

H

Hydrometric Service

Office of Public Works

17/19 Lower Hatch St.

Dublin 2

C

Ordinance Survey

Phoenix Park

Dublin 8

Assistant Director: Commdt. M. C. Walsh

Petroleum and Minerals Division

Department of Industry, and Energy

Nassau House

Dublin 2

Principal Officers: P. O’Brien

M. O'Connell

H

$\mathrm{H}$

$\mathrm{C}, \mathrm{G}, \mathrm{H}, \mathrm{R}$

Tahal-Water Planning for Israel

P.O.'Box 11170

Tel Aviv

Director General: Arieh Gissem

Oceanographic and Limnological Research Institute Tel Shikmona, Haifa

P.O. Box 8030

Haifa

Director: Dr. Collette Zruia

Earth-Sciences Research Administration

Ministry of Energy and Infrastructure

P.O. Box 1442

jerusalem

Director: Dr. Michael Beyth 
Direzione Generale delle Miniere e della Geologia Ministero dell'Industria e del Commercio

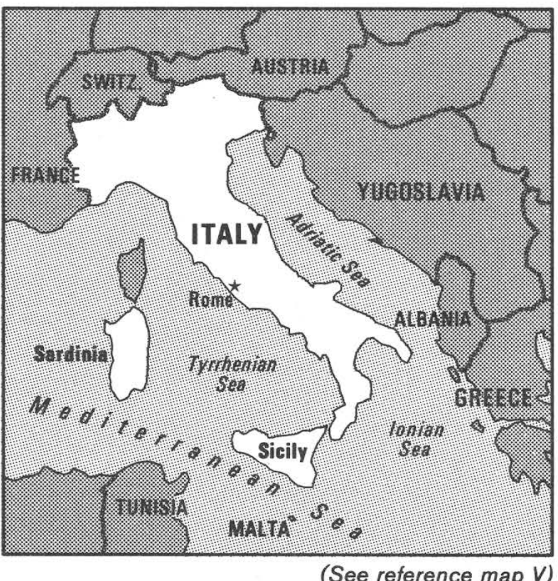

Via Molise 2

00187 Roma

Director General: Ing. Silvano Santini

\section{IVORY COAST}

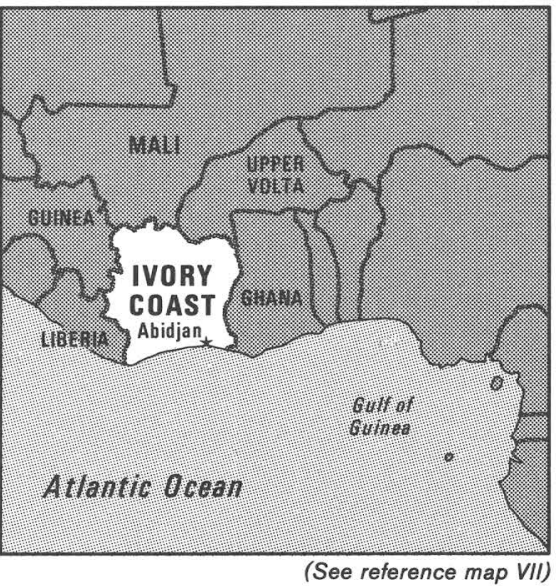

Istituto Internazionale per le Richerche Geotermiche

Via del Buongusto 1

56100 Pisa

Director: Prof. Learco Taffi

Istituto Internazionale di Vulcanologia

Viale Regina Margherita 6

95123 Catania

Director: Prof. Letterio Villaria

Osservatorio Vulcanologico Vesuviano

80056 Ercolano (Napoli)

Director: Prof. Paolo Gasparini

R,C,G Ministère des Mines

B.P. V 50

Abidjan

Minister: Paul Gui Dibo

Direction des Mines et de la Géologie Ministère des Mines

B.P. V 81

Abidjan

Director: Jean Yagui Likan

H Servizio Idrografico Centrale

Ministero dei Lavori Pubblici

Via Nomentana 2

00161 Roma

Director: Dr. Bruno Travaglini

Istituto Geografico Militare

Via Cesare Battisti 10

50122 Firenze

Director: Gen. Luigi Zanetti

H Istituto Idrografico della Marina

Passo Osservatorio 4

161134 Genova

Director: Capt. Allesandro Ronca

Comitato Nazionale per le Scienze Geologiche e Minerarie

Consiglio Nazionale delle Ricerche

Piazzale Aldo Moro 7

00185 Roma

President: Prof. Antonio Praturlon

Direction des Hydrocarbures

Ministère des Mines

B.P. V 42

Abidjan

Director: René Brancart

Direction de la Géologie

Ministère des Mines

B.P. V 28

Abidjan

Director: Victor Sea

G,C Société pour le Développement Minier de la Côte d'Ivoire (SODEMI)

B.P. 2816

Abidjan

Director: Joseph N'zi

G, R Société Nationale d'Opérations Petrolieres de la Côte d'Ivoire (PETROCI)

Ministère des Mines

B.P. V 194

Abidjan

Director-General: Paul Ahui 
Abidjan

C

Institut Géographique de Côte d'Ivoire (IGCI)

Ministere des Travaux Publics et des Transports 01 BP 3862

Abidjan 01

Director: Alpha Cissé

H

Société pour la Realisation de Forages d'Exploitation en Côte d'Ivoire

Ministère du Plan

B.P. V 65

Abidjan

Director: Antoine Kouao

\section{JAMAICA}

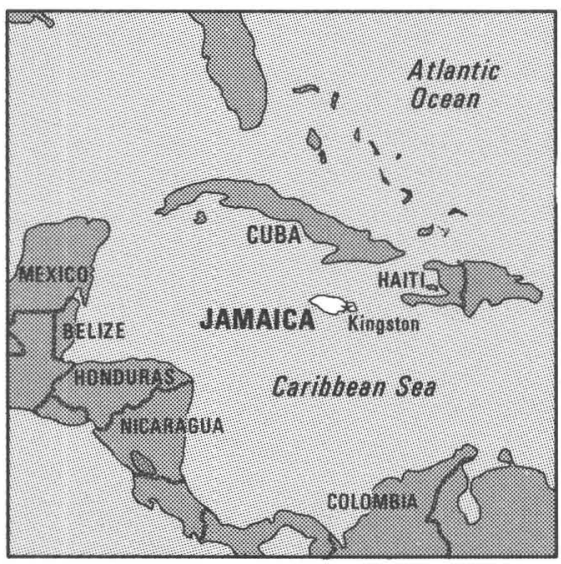

(See reference map III)

G

$\mathrm{H}$

Geological Survey Division

Ministry of Mining and Natural Resources

Hope Gardens

Kingston 6

Acting Director: Godfrey Perkins

Water Resources Division

Ministry of Local Government

P.O. Box 91, Hope Gardens

Kingston

Director: Serrant Hardware

C

Survey Department

P.O. Box 493

Kingston

Acting Director of Surveys: Rupert Robinson

National Water Authority

14-19 Trinidad Terrace

Kingston 5

Managing Director: S. A. Small
Mines and Quarries Division

Ministry of Mining and Natural Resources

Hope Gardens

Kingston 6

Commissioner of Mines:. Godfrey Perkins

$\mathbf{R}$

Petroleum Corporation of Jamaica

2 King St.

Kingston

Managing Director: William Saunders

\section{JAPAN}

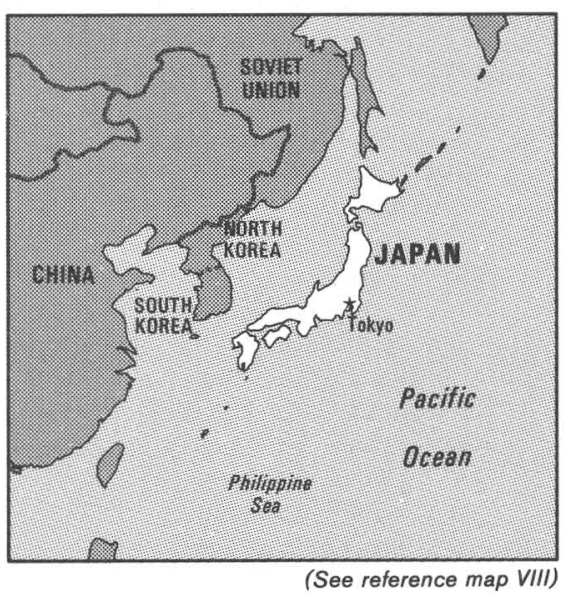

G

G,H

G

G

\section{Nippon Kazan Gakkai}

[Volcanological Society of Japan]

Earthquake Research Institute

University of Tokyo

1-1 Yayoi-cho 1-chome

Bunkyo-ku, Tokyo 113

President: Joyo Ossaka

National Institute of Resources

Science and Technology Agency

2-2-1 Kasumigaseki

Chiyoda-ku, Tokyo 100

Director: Kiichiro Nagara

Public Works Research Institute

Ministry of Construction (includes engineering geology)

1, Asahi, Toyosato-machi

Tsukuba-gun, Ibaraki-ken 305

Director:. Ryuichi Iida

Earthquake Research Institute

University of Tokyo

1-1-1 Yayoi-cho 1-chome

Bunkyo-ku, Tokyo 113

Director: Daisuke Shimozuru 
Geological Survey of Japan, Ministry of International Trade and Industry (MITI)

1-1-3 Higashi, Yatabe, Tsukuba-gun, Ibaraki-ken 305

Director: Junji Suyama

G Agency of Natural Resources and Energy Ministry of International Trade and Industry (MITI)

1-3-1 Kasumigaseki

Chiyoda-ku, Tokyo 100

Director-General: Toru Toyoshima

G,R National Research Institute for Pollution and Resources

Ministry of International Trade and Industry (MITI)

Onogawa Yatabe-machi,

Tsukuba-gun, Ibaraki-ken 305

Director: Toru Ishihara

$\mathrm{H}$

River Bureau

Ministry of Construction

2-1-3 Kasumigaseki

Chiyoda-ku

Tokyo 100

Director-General: Masatomo Kawamoto

H Hydrographic Department

Maritime Safety Agency

Ministry of Transport

5-3-1, Tsukiji

Chuo-ku, Tokyo 104

Director-General: Kuniro Sugiura

H Water Resources Bureau

National Land Agency

Prime Minister's Office

1-6-19 Azabudai

Minato-ku, Tokyo 106

Director-General: Hidenobu Takahide

C Geographical Survey Institute

Ministry of Construction

1, Kitasato, Yatabe-cho

Tsukuba-gun, Ibaraki-ken 305

Director-General: Minoru Tajima

$\mathbf{R}$

Technology Research Center

Japan National Oil Corporation

2-2-2 Uchisaiwai-cho, Chiyoda-ku, Tokyo 100

Director: Masakazu Kato

\section{JORDAN}

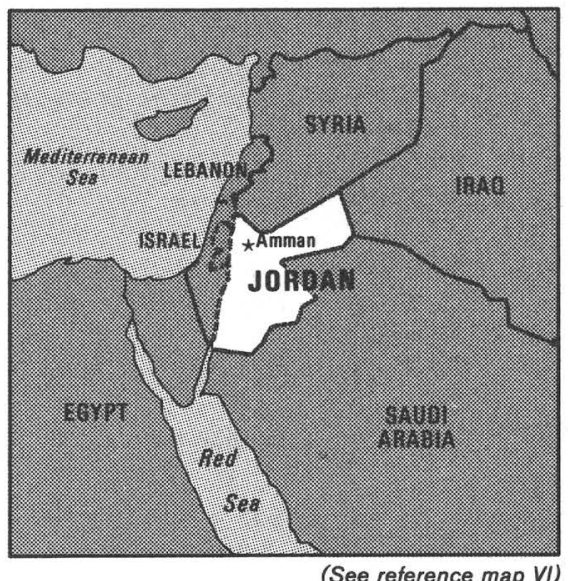

G

Department of Geological Resources and Mining (NRA)

P.O. Box 39

Amman

Director: Mohammed Abu Ajamiyeh

G

Royal Scientific Society

P.O. Box 6945

Amman

President: Albert Butros

G,C,H, R Natural Resources Authority

P.O. Box 7, 39, or 2220

Amman

Vice-President: Ahmad Dukhgan

C

Department of Lands and Surveys

Ministry of Finance

P.O. Box 70

Amman

Director: Badri Al-Mulqi

C Jordan National Geographic Center

P.O. Box 20214

Amman

Director: Rafat Majali 


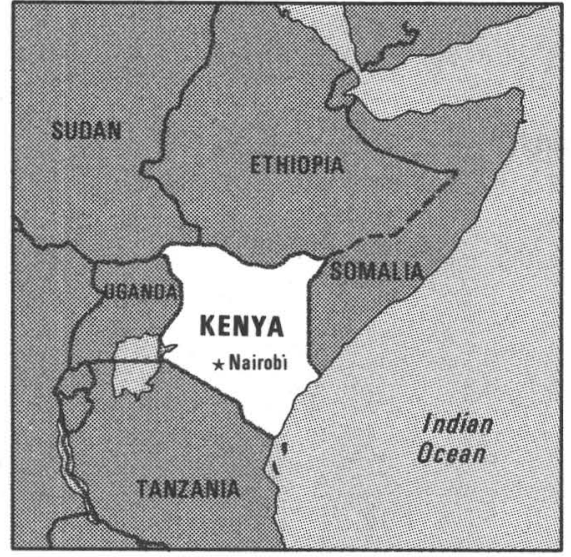

(See reference map VII)

G,R Geological Survey of Kenya

Mines and Geological Department

Ministry of Natural Resources

Madini House, Machakos Road

P.O. Box 30009

Nairobi

Chief Geologist: J. K. Wachira

G

Department of Geology

University of Nairobi

P.O. Box 30197

Nairobi

Chairman: Dr. I. O. Nyambok

C Survey of Kenya

P.O. Box 30046

Nairobi

Director of Surveys: D. Kamau

$\mathrm{H}$

Water Department

Ministry of Water Development

P.O. Box 30521

Nairobi

Director: Water Eng. Dept. - C. N. Mutitu

Water Res. Dept. - D. N. Kirori

$\mathbf{R}$
Ministry of Environmental and Natural Resources P.O. Box 30126

Nairobi

Deputy Secretary: G. G. Njoroge

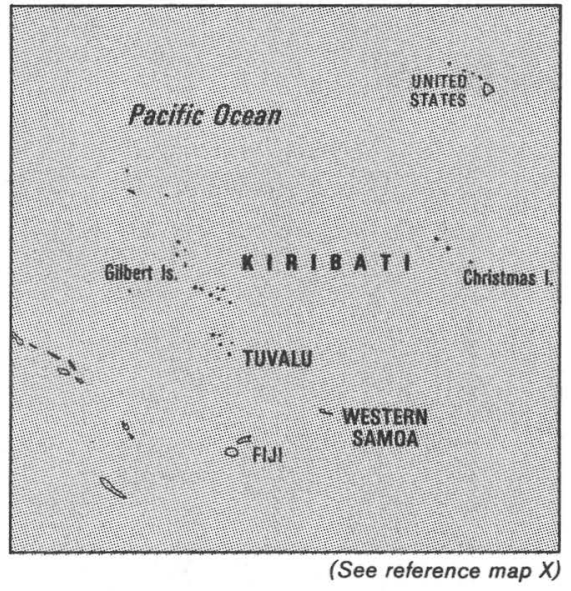

G

Ministry of Local Government and Rural Development

Lands and Survey Division

P.O. Box 405

Betio, Tarawa

$\mathrm{H}$

Ministry of Natural Resources

P. O. Box 64

Bairiki, Tarawa

Secretary for Natural Resource: M. Irata

\section{KOREA, NORTH}

(No recent information)

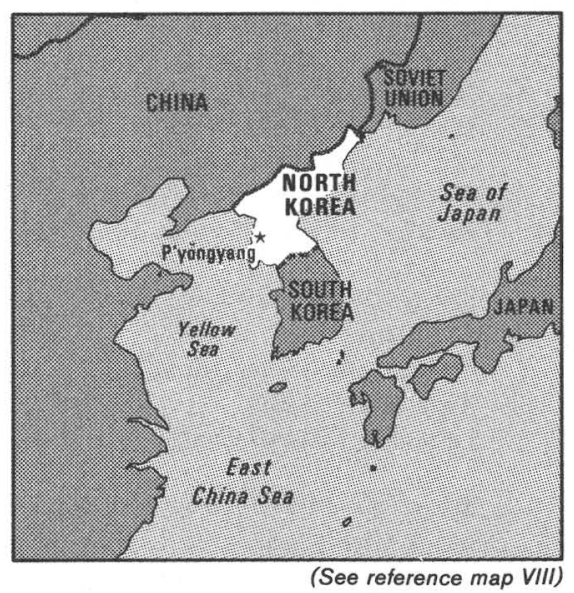

C

Geology and Geography Research Institute

Academy of Sciences

Mammoon-dong, Central District

P'yongyang

Director: Pak Sung Wook

$\mathrm{H} \quad$ Hydro-Meteorological Bureau

P.O. Box 100

P'yongyang

Director: Li Gon I1 


\section{KOREA, SOUTH}

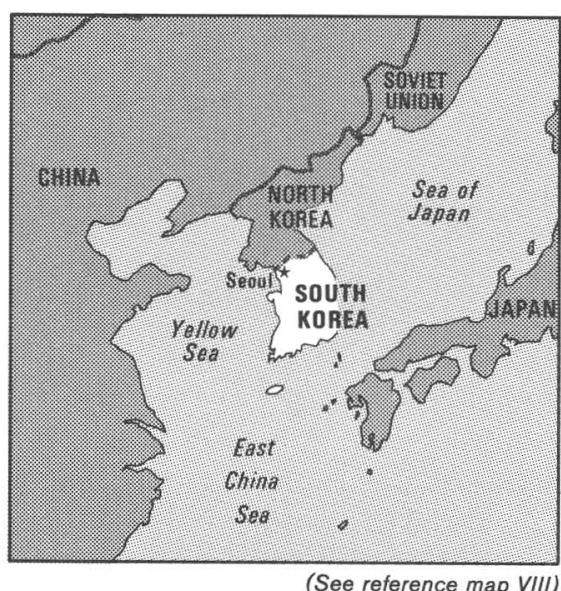

(See reference map VIII)

G

G,C Korea Institute of Energy and Resources (KIER)

219-5, Garibong-dong

Guro-Gu, Seoul

President: Park Keung-Shik

H,C Hydrographic Office

Ministry of Transportation

355 , Chungrim-dong

Chung-Gu, Seoul

Director General: Kim Mun-Son

R

$\mathrm{H}$

Korea Mineral and Mining Development

Cooperation

48-686-1, Sindaebang-dong

Kwanak-Gu, Seoul

President: Kim Bok-Dong

Korea Ocean Development Research Institute 215-1, Socho-dong

Kangnam-Gu, Seoul

Director General: Huh Hyung-Dack

C

Korean Society of Geodesy, Photogrammetry and Cartography

18, Kyonam-dong

Jongro-Gu, Seoul

President: Ahn Chul-Ho

\section{KUWAIT}

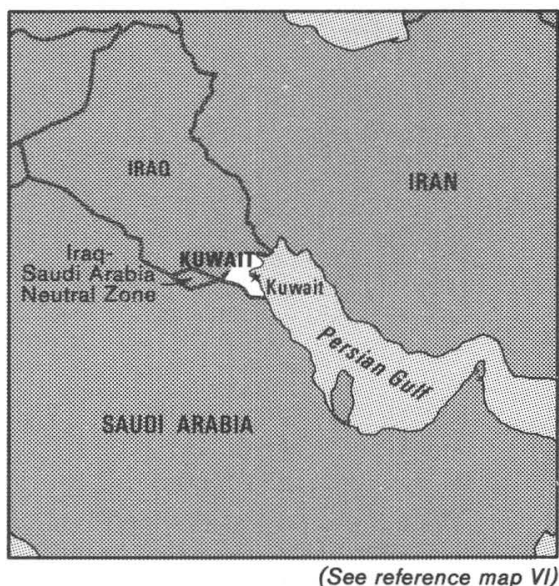

G P.O. Box 12009 Shamiah

Kuwait

Director-General: Adnan Shehabedin

Ministry of Electricity and Water

P.O. Box 12

Kuwait

Head of the Design Section of Water Department: Mustapha Al Shater

Director of Building and Survey Department Kuwait Municipality

P.O. Box 10, Safat

Kuwait

Director and Chief Engineer: Hamed A. Shuaib

Ministry of Oil

P.O. Box 5077

Kuwait

Assistant Secretary for Technical Affairs: Sulaiman N. Al-Omani

Kuwait National Petroleum Company

P.O. Box 70

Kuwait

Chairman and Managing Director: Ahmad Abdulmohsin Al Mutair 
Ministry of Hydro-Electric Resources

Beirut

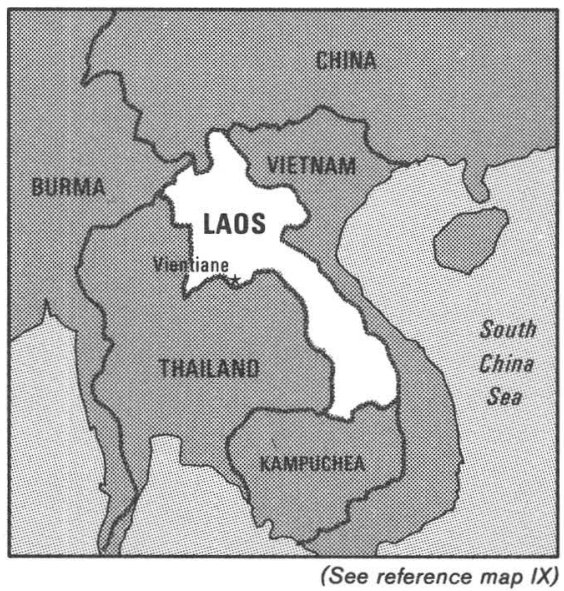

Director General of Hydro-Electric Installation: Rida Dehaini

Director of Hydraulic Installations: Lucien Mubayed

$\mathbf{R}$

Ministry of Petroleum

Beirut

Director General: Ibrahim Choueiri

R

Ministry of Industry

Beirut

Director, Technical Department: Imad Nawam

\section{LESOTHO}

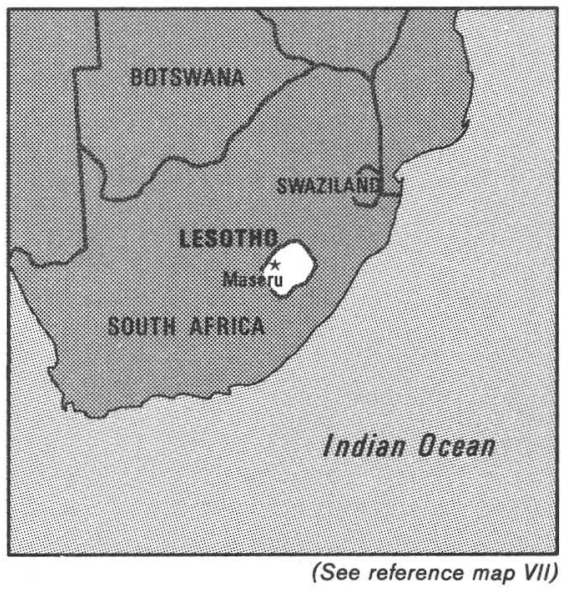

Vientiane

Director of Cartography: Kone Keognothi

\section{LEBANON}

(No recent information)

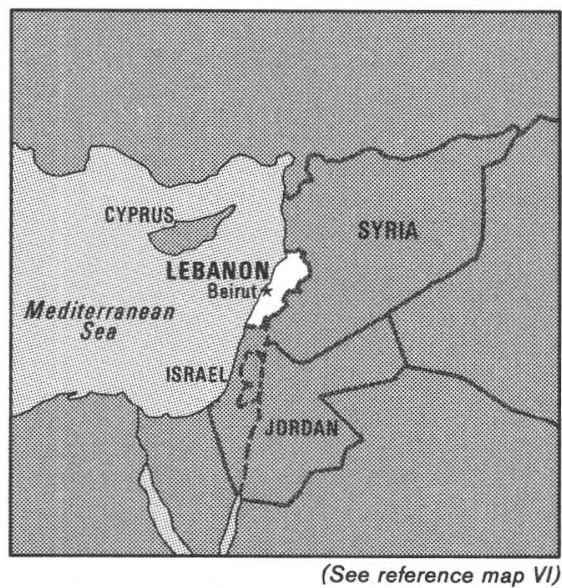

Directorate General of Public Works

Ministry of Public Works

Beirut

Director General: Antoine Rayes
G

Department of Mines and Geology Ministry of Water, Energy and Mining

P.O. Box 750, Maseru 100

Commissioner: M. Lerotholi

C

Department of Lands, Surveys and Physical Planning

Ministry of Interior, Chieftainship Affairs and Social Welfare

P.O. Box 174, Maseru 100

Commissioner: Abner Mosaase

Hydrology and Meteorological Services Ministry of Water, Energy, and Mining P.O. Box 772, Maseru 100

Principal Hydrologist: Sechocha Makhoalibe Acting Principal Hydrologist: Tseliso Sekoli 


\section{LIBERIA}

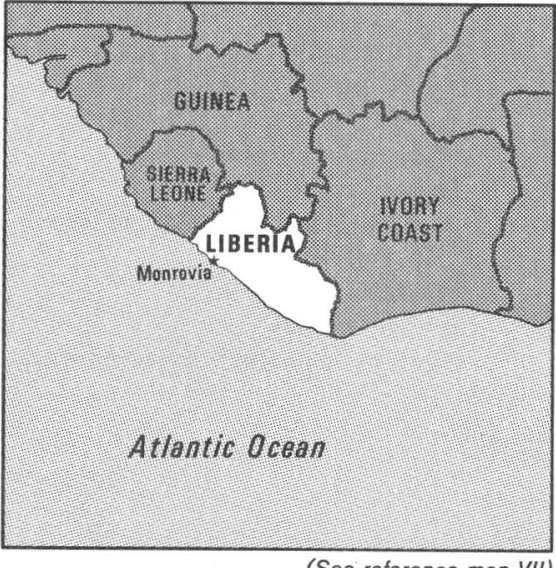

G,C,H, R Ministry of Lands, Mines and Energy

Capitol Hill

P.O. Box 9024

Monrovia

Minister: Fodee Kromah

G

Liberian Geological Survey

Ministry of Lands, Mines and Energy

P.O. Box 9024

Monrovia

Director: Dr. Eugene Shannon

C

Liberian Cartographic Service

Ministry of Lands, Mines and Energy

P.O. Box 9024

Monrovia

Director: G. Yarkpazuo Miller

H Liberian Hydrological Service

Ministry of Lands, Mines and Energy

P.O. Box 9024

Monrovia

Director: John Nylander

G, R Bureau of Mines

Ministry of Lands, Mines and Energy

P.O. Box 9024

Monrovia

Director: Amara Bako Freeman

R Bureau of Hydrocarbon

Ministry of Lands, Mines and Energy

P.O. Box 9024

Monrovia

Director: Dr. E. Miedi Himie Neufville

$\mathbf{R}$
Bureau of Lands, and Survey

Ministry of Lands, Mines and Energy

P.O. Box 9024

Monrovia

Director: James G. Glay

\section{LIBYA}

(No recent information)

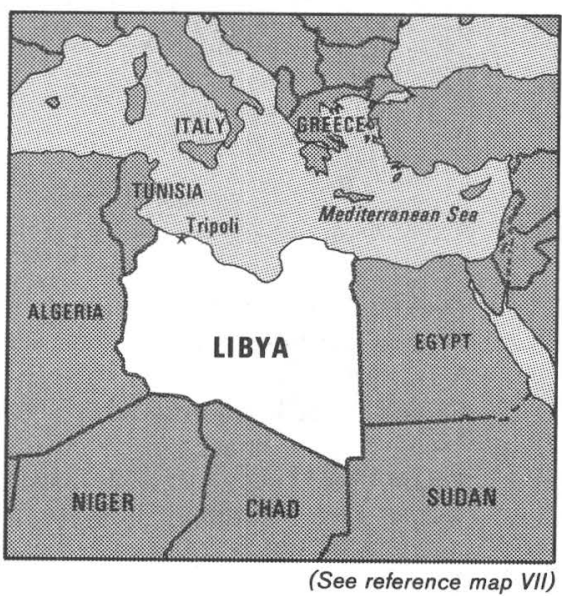

G

G, C, H, R Planning Division

Secretariat of Agrarian Reform and Land Reclamation

P.O. Box 190

Tripoli

Director: Ahmad Gartaa

G,H Agricultural Research Center

P.O. Box 2480

Tripoli

Chairman: Abdoulmajid Bensad

G,H,C, R Geological Department

Faculty of Science

Al-Fateh University

P.O. Box 398

Tripoli

$\mathrm{H}$

Water Resources and Dams Ministry

Tripoli

$\mathrm{H}$

Water Department

Secretariat of Agrarian Reform and Land

Reclamation

P.O. Box 190

Tripoli

Director: Abdullah M. Krewi 
C

Department of Surveying

Secretariat of Planning

P.O. Box 600

Tripoli

Director: Muftah M. Unis

R Secretariat of Petroleum

Tripoli

Minister: Izziddin Mabruk

\section{LIECHTENSTEIN}

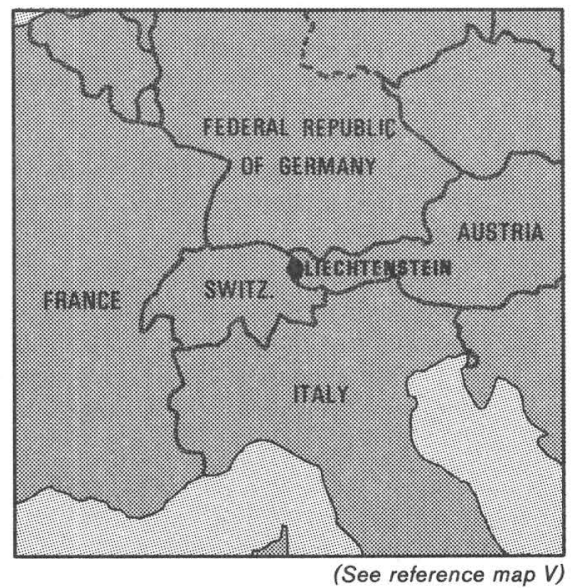

G,C

Landesbauamt des Fürstentums Liechtenstein

Städte 49

9490 Vaduz

Chief Surveyor: Dr. Karl Hartmann

$\mathbf{R}$

Amt. für Gewässerschultz

[Water Conservation Office]

Städtle 49

9490 Vaduz

Director:. Theo Kindle

$\mathrm{C}, \mathrm{R}$

Landwirtschaftamt

[Agricultural Office]

Städtle 49

9490 Vaduz

Director: Ernst Ospelt

\section{LUXEMBOURG}

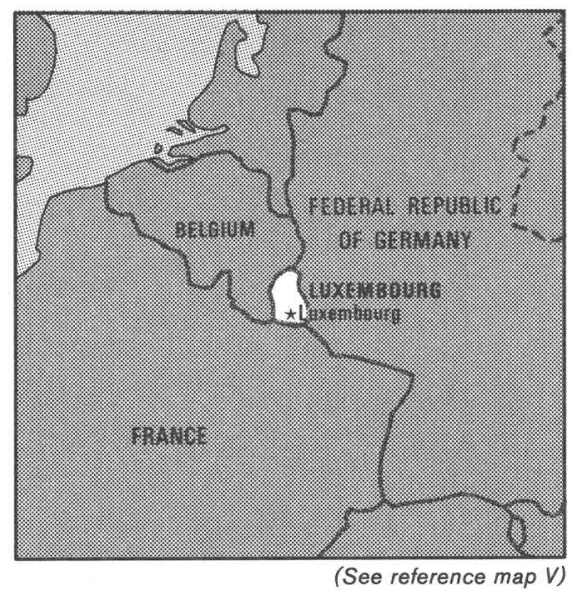

G

Centre Séismologique

Walferdange

Administration Communale

Service Topographique

51, Bol. Royal

Luxembourg

Director: Jean Flick

G

Service Géologique

Ponts et Chaussées

43. Bol. G.D.-Charlotte

Luxembourg

Director: Jacques Bintz

$\mathrm{H}$

Service de la Metéorologie et de l'Hydrologie

16, route d'Esch

Luxenbourg

Director: R. Kipgen

C

Administration du Cadastre et de la Topographic 54 Avenue Gaston Diderich

Luxembourg

Director: Victor Poos 


\section{MADAGASCAR}

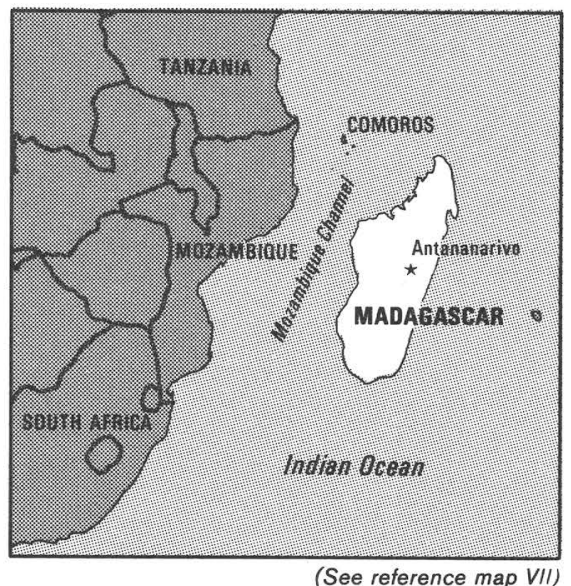

G,E,C, R Ministere de l'Industrie, de l'Energie at de Mines (MIEM)

B.P. 280

Antananarivo

Minister: Tantely Andrianarivo

Director General: Blandin Razafimanjato

$\mathbf{R}$

G, $R \quad$ Chief of Service of Mines and Geology: Jean Rene S. Ratsimbazafy

G

$\mathrm{H}$

$\mathrm{G}, \mathrm{H}$

G

G

Chief of Division of Geology: Jeannot Rasoanaivo

Chief of Service of Energy: Ammanuel Randrianariosoa

\section{Chief of Division of Hydrogeology:} Jean Herivelo Rakotondrainibe

Ministere de La Recherche Scientifique et B.P. 694

Antananarivo

Minister: Zafera Antoine Rabesa

Secretary General: Henri Rasolondraibe

\section{MALAWI} Technologie pour le Developpement (MRSTD

Centre National de Recherches Oceanographiques B.P. 68

Nosy Be

Director: Andrianirina Ralison
Institut National de Géodesie et de Cartographic [Foibe-Taosarintanin'i Madagasikara (FTM)] 3 Lalana J. H. Ravelomanantsoa

B.P. 323, Andohalo

Antananarivo

Director: M. S. Andriamihaja

Service de la Meteorologie Direction des Transports et de la Meteorologie, MTRT

\section{B.P. 125}

Chief of Service: Elie Randrianarison

Division de l'Hydrologie

Chief of Division: Andriambola Ralison

Office Militaire National Pour les Industries Strategiques (OMNIS)

B.P. 1 bis

Antananarivo

Director General: General Hubert Andrianasolo Director of Atomic Energy:

Andrianaivomanana Razafindramisa

Director of Hydrocarbons: Georges Ramanoara

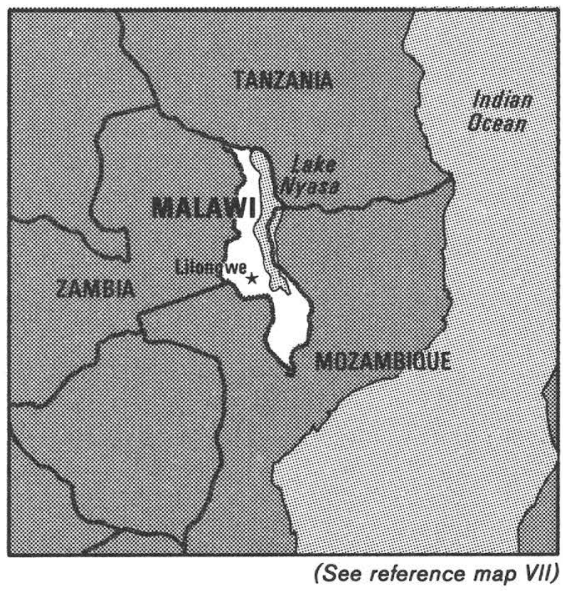

G,H Geological Survey Department Ministry of Natural Resources P.O. Box 27, Liwonde Road Zomba Chief Geologist: Dr. R. L. Johnson 
Ministry of Natural Resources

P.O. Box 349

Blantyre

Chief Cargographer: A. F. Tambala

$\mathrm{H}$

Water Resources Division

Ministry of Agriculture

P.O. Box 30026

Lilongwe 3

Chief Water Resources Officer: K. A. Edwards

Senior Hydrologist: L. Z. Laisi

\section{MALAYSIA}

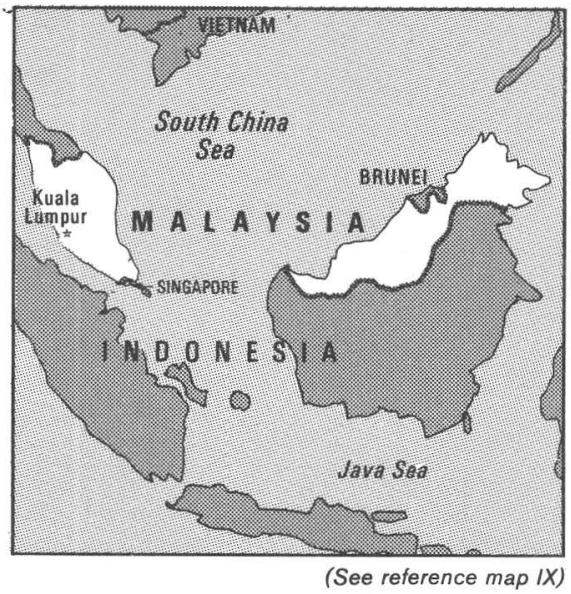

G

Geological Survey Department

Bangunan Ukor, 2d Floor

Jalan Gurney

Kuala Lumpur

Director-General: Dr. Santokh Singh

$\mathrm{H}$

Drainage and Irrigation Department

Ministry of Agriculture

Jalan Mahameru

Kuala Lumpur, Malaysia 10-02

Director General: Pang Leong Hoon

C

Directorate of National Mapping

Bangunan Ukor, 4th Floor

Jalan Gurney

Kuala Lumpur

Director: Seah Kok Seang
$\mathbf{R}$

Petronas [National Petroleum Company]

P.O. Box 2444

Kuala Lumpur

Chairman: Tan Sri Abdullah

Bin Mohd. Salleh

R

Mines Department

Bangunan Ukor, 6th Floor

Jalan Gurney

Kuala Lumpur

Head: Tan Kim Bee

\section{MALI}

(No recent information)

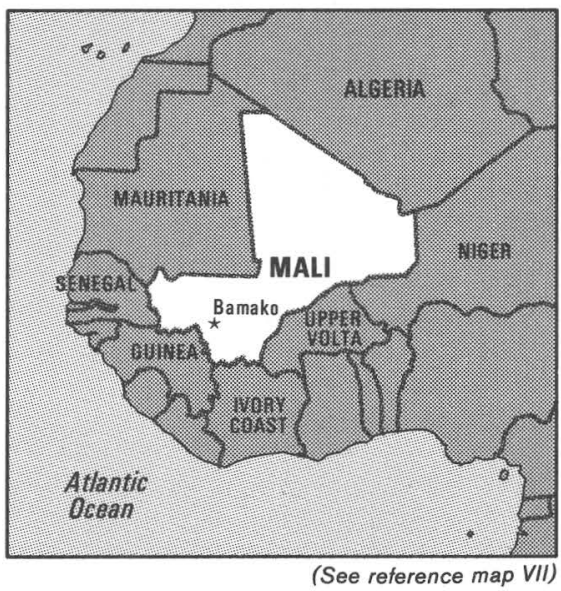

G

Direction Nationale des Mines et de la Geologie B.P. 223

Koulouba, Bamako

Directeur General: Sekou Diallo

C Institut National de Topographie

B.P. 240

Bamako

Director: Diadie Traore

H

Service Hydrologique

Direction Generale de l'Hydraulique et de

B.P. 66 l'Energie

Bamako

Chief: Dembelle Aly

$\mathbf{R}$
Société Nationale de Recherches et d'Exploitation des Resources Minières du Mali (SONAREM)
B.P. 2
Kati
Directeur General: Makan Kayentao




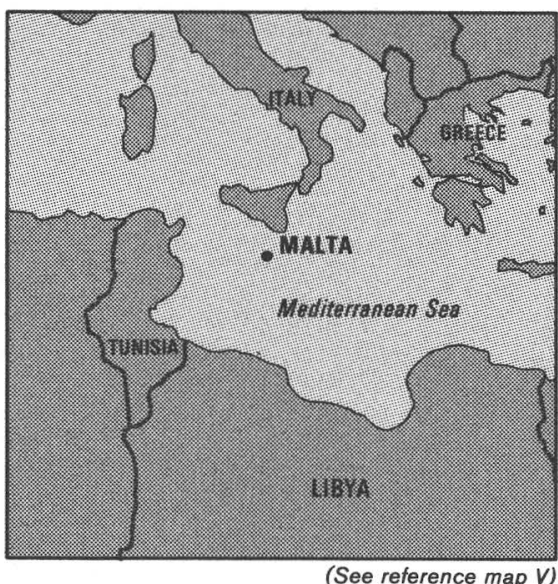

G

H

C

R
Public Works Department

Beltissebh

Hydrology Section

Water Works Department

Beltissebh

Floriana

Chief: Francis Toledo

Information Division

Auberge de Castille

Valletta

Ministry of Parastatal and People's Investments

Lascaris

Valletta

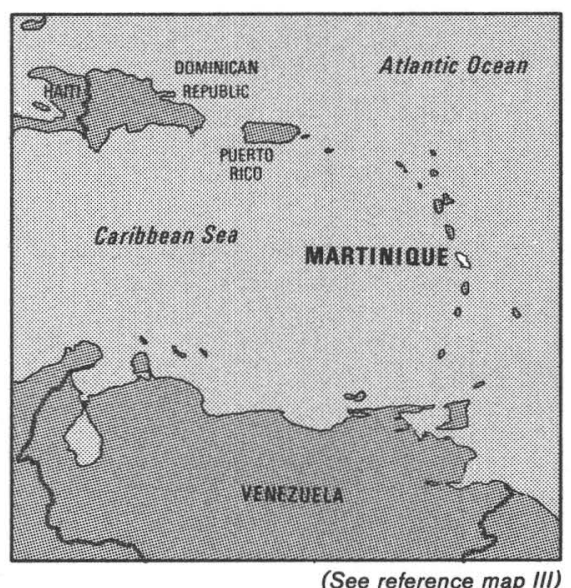

$\mathrm{G}, \mathrm{H}$

Office de la Recherche Scientifique et Technique Outre-mer (ORSTOM) ${ }^{7}$

B.P. 81

97000 Fort-de-France

\section{MAURITANIA}

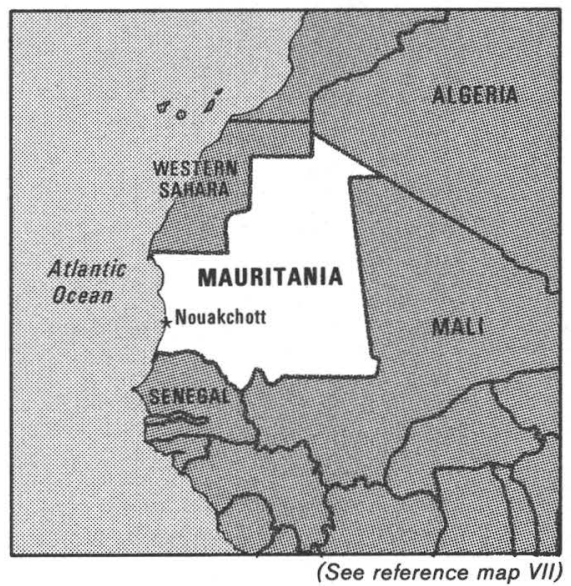

G

Direction des Mines et de la Géologie Ministère des Mines et de l'Energie B.P. 199

Nouakchott

Director: Abdel-Kader Ould Saleh

G

Office Mauritanien de Recherches Géologiques

B.P. 654

Nouakchott

Director: Cheikhouna Kamara

\footnotetext{
${ }^{7}$ French research organization; no governmental affiliation.
} 


\section{MAURITIUS}

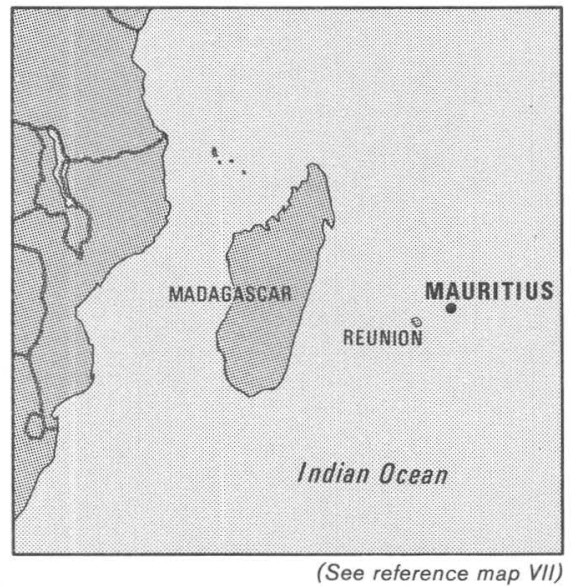

Ministry of Agriculture and Natural Resources and The Environment

Port Louis

Ministry of Housing, Lands, and Town and Country Planning

Survey Department

Port Louis

Chief Surveyor:. M. Derblay

Central Water Authority

Jules Koenig Street

Port Louis

Principal Hydrologist: G. Rogbeer

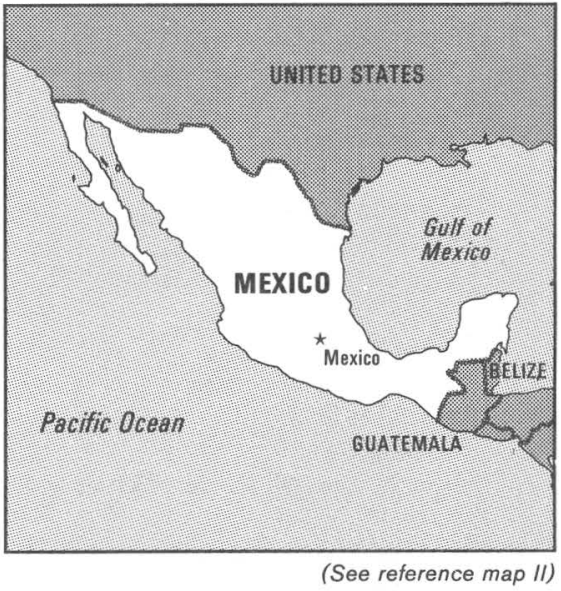

Instituto de Géologia

Universidad Nacional Autónoma de Mexico (UNAM)

Cuidad Universitaria

México 20, D.F.

Director: Dr. José Guerrero García

Instituto de Biología

Universidad Nacional Autónoma de México (UNAM)

Ciudad Universitaria

México 20, D.F.

Director: Dr. José Sarukhán Kermez

Consejo de Recursos Minerales Niños Heroes 139, Esq. Dr. Navarro

México 7, D.F.

Director General: Jorge Leipen Garay

G

Coordinación de la Investigación Científica

Universidad Nacional Autónoma de México (UNAM)

Ciudad Universitaria

México 20, D.F.

Coordinador: Dr. Jaime Martuscelli

G

Instituto de Ciéncias del Mar y Limnología Universidad Nacional Autónoma de México (UNAM)

Ciudad Universitaria

México 20, D.F.

Director General: Dr. Agustín Ayala Castañares

G

Comision de Fomento Minero

Puente de Tecamachalco No. 26

México 10, D.F.

Apartado Postal 10-762

Director General: Lic. Luís de Pablo Ferna 
Instituto de Geofísica

Universidad Nacional Autónoma de México (UNAM)

Ciudad Universitaria

México 20, D.F.

Director: Dr. Ismael Herrera Revilla

G

Instituto Mexicano de la Fluorita, A.C.

Tokio No. 4, Esq. Praga

Apartado Postal 6-783

México 6, D.F.

Director General: Ing. Alejandro Prieto Huesca

G

Petroleos Mexicanos

Av. Marina Nacional 329

Edificio $1810,12^{\circ}$ piso

México 17, D.F.

Director General: Lic. Mario Ramón Beteta

G

Instituto Mexicano del Petróleo

Av. Cien Metros No. 152

México 14, D.F.

Director General: Ing. José Luis García Luna Hernández

G

G

G

C,G Departmento Geográfico Militar

Servicio Cartográfico

Secretaria de la Defensa Nacional

Lomas de Sotelo

México 10, D.F.

Director General: General Ulises Beltran Tenorio

C,G Dirección General de Estudios del Territorio Nacional (DETENAL)

Secretaria de Programacion y Presupuesto

San Antonio Abad $124,5^{\circ}$ piso

México 8, D.F.

Director: Lic. Nestor Duch Gary

$\mathrm{H}$
MONGOLIA

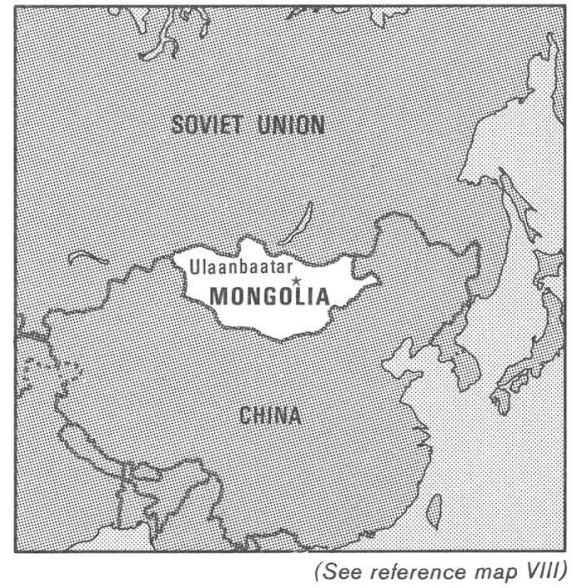

G

Institute of Geology

Academy of Sciences

UI, Leniadom 2

Ulaanbaatar

Director: B. Luvsandandzan

G,C Institute of Geography and Permafrost Studies Academy of Sciences

Ulaanbaatar

Director: S. H. Tsegmed

G,H Hydrometeorological Service

Gudamj Ooctober 5

Ulaanbaatar

Director: D. Tubdendorf

$\mathrm{H}$

Ministry of Water Economy

Gudamj Natsagdorjyn

Ulaanbaatar 


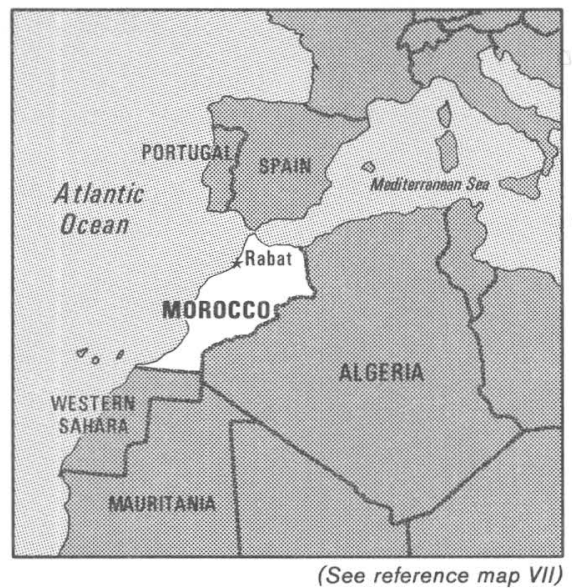

G Division de la Géologie

Ministère de l'Energie et des Mines

Quartier Administratif

Rabat

Director: Mohamed Ben Said

Direction de la Recherche Agronomique

Service Cartographique des Sols et de l'Erosion

Avenue de la Victoire

B.P. 415

Rabat

Chief: M. H. Ghanem

C

Ministère de l'Agriculture et de la Reforme Agraire

Quartier Administratif

Rabat

Minister: Othman Demnati

H Direction de l'Hydraulique

Division de Ressources en Eau

Avenue John Kennedy

B.P. 525 Rabat-Chellah

Rabat

Director: Abdelhaq Fakihani

$\mathrm{R}$

Ministere de l'Energie et des Mines

Ministry of Energy and Mines

Quartier Administratif

Rabat

Minister: Moussa Saaid

Director of Mines: Othman Kettouche

Director of Energy: Ahmed Bouhouli

R

Societe Nationale des Ressources Pétrolières (SNPP)

42, avenue de l'Armée Royale

3 ème étage

B.P. 178

Casablanca

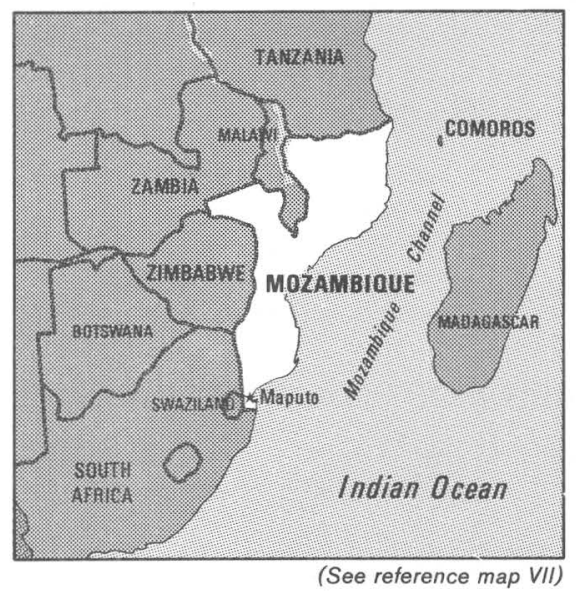

G,R Direccão Nacional de Geologia [National Directorate of Geology]

Praca 25 de Junho/Maputo

P.O. Box 217

Maputo

Director: Rui Santos Garcia

Direccão Nacional de Minas

[National Directorate for Mines]

Avenida Filipe Samuel Magaia, 528

2d Floor

P.O. Box 217

Maputo

Director: Dr. Carlos Magno

R

Empresa Nacional de Minas E.E.

[National Mining Enterprise]

Avenida 24 de Julho 1895, 2d Floor

P.O. Box 1152

Maputo

Director: Dr. Mahomed Jaffarullah

R

Secretariado de Estado do Carvao e

Hidrocarbonetos

[State Secretariat for Coal and Hydrocarbons] Avenida Samora Machel, 39

P.O. Box 2904

Maputo

Secretary of State: Abdul Magid Osman

Direccão Nacional de Águas

[National Directorate of Hydrology]

Avenida 25 de Setembro, 942 - 7th Floor/Maputo

P.O. Box 1611

Maputo

Director: Eng. Arnaldo Pereira 
[National Directorate for Geography and Mapping]

Avenida Josina Machel, 589/Maputo

P.O. Box 288

Maputo

$\mathbf{R}$

Ministry for Mineral Resources

Praca 25 de Junho

P.O. Box 217

Maputo

Minister: José Carlos Lobo

\section{NAMIBIA (SOUTHWEST AFRICA)}

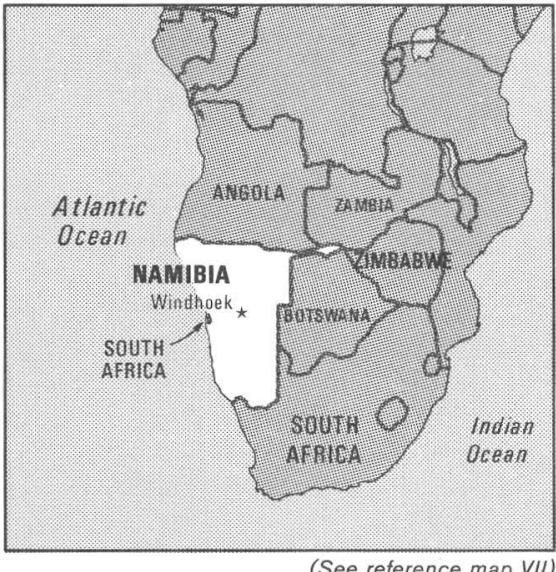

G

Geological Survey

P.O. Box 2168

Windhoek

C
NEPAL

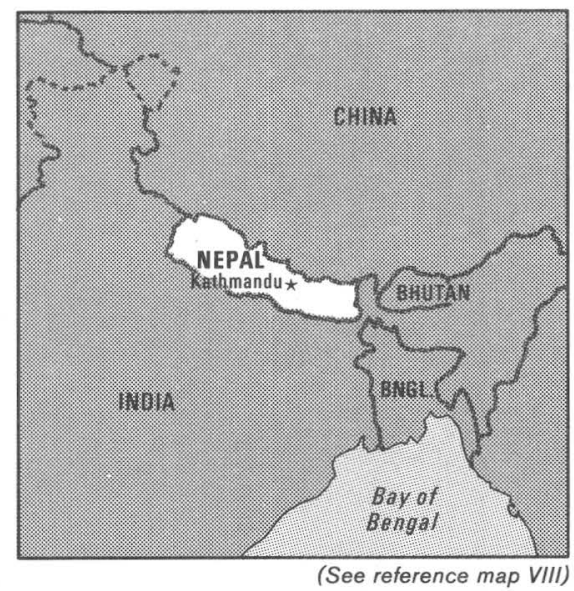

G, R Department of Mines and Geology

Ministry of Industry

Lainchaur

Kathmandu

Director-General: Mahendra Narsingh Rana

C

Survey Department

Ministry of Land Reform

Dilli Bazar

Kathmandu

Director General: Arjun Bahadur Basnyat

$\mathrm{H}$

Department of Irrigation, Hydrology and Meteorology

Ministry of Water Resources

Panipokhari

Kathmandu

Director-General: Chitra Dev Bhatta

\section{NETHERLANDS, THE}

P/B 13182 Kaiser Street

Windhoek

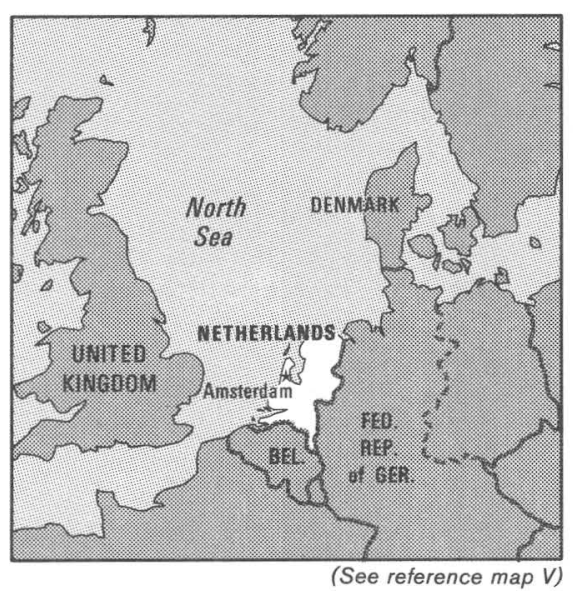

Rijks Geologische Dienst

Spaarne 17, P.O. Box 157

2000 AD Haarlem

Director: B. P. Hageman 
2501 BD 's-Gravenhage

Groundwater Survey TNO

P.O. Box 285

2600 AG Delft

Director: F. Walter

H Ministry of Transport and Water Resources

Rijkswaterstaat [State Public Works]

Directorate for Water Management and Hydraulic Research

1 Hooftskade

2526 KA The Hague

Dienst van het Kadaster

Waltersingel 1

7314 NK Apeldoorn

Topografische Dienst

Westvest 9

2611 AX Delft

Ministry of Economic Affairs

Directorate General for Energy

Bezuidenhoutseweg 30

2594 AV The Hague

Director General: Dr. G. H. B. Verberg

\section{NETHERLANDS ANTILLES}

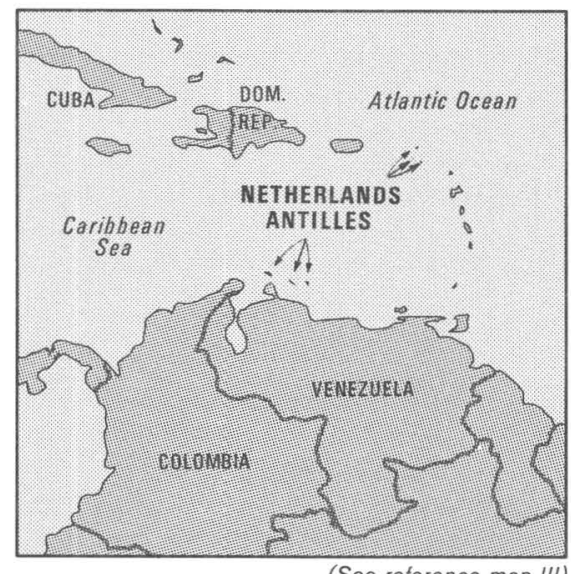

G

Oranjestad, Aruba

Director: T. M. Monzon

\section{NEW CALEDONIA}

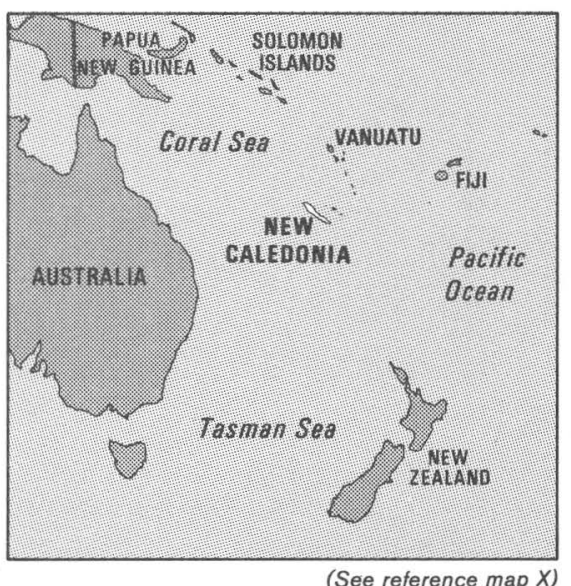

Office de la Recherche Scientifique et Technique Outre-mer (ORSTOM) ${ }^{8}$

B.P.A. 5

Noumea Cedex

Chief Scientist: J. Recy

Service des Mines et de la Géologie

Rte. No. 1

B.P. 465

Noumea

C Service Topographique

Territorial Administration Center

Avenue Paul Doumer

Noumea Nouvelle

\footnotetext{
${ }^{8}$ French research organization; no governmental affiliation.
}

Bureau voor Kadaster en Kaartering

Sint Anna Boulevard 4

Willemstad, Curacao

Director: Ir. H. B. Calvo

$\mathrm{R}$

Bureau voor Olie Aangelegenheden

[Bureau of Petroleum Affairs]

Wolkstraat 3

Willemstad, Curacao

Director: Clark Gomes-Casseres 
NEW ZEALAND

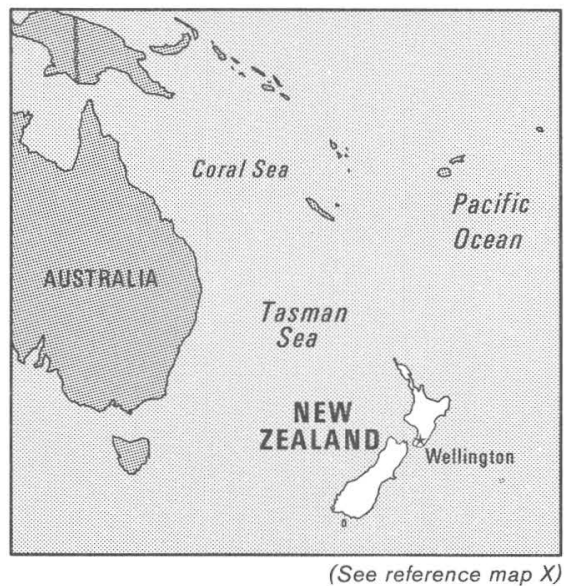

G

Geophysics Division

Department of Scientific and Industrial Research

P.O. Box 1320

Wellington 1

Director: Dr. T. Hatherton

G

C

$\mathrm{H}$

$\mathrm{H}$

$\mathbf{R}$

P.O. Box 30-368

Lower Hutt

The Surveyor General, Head Office

Department of Lands and Survey

Private Bag, Charles Ferguson Building

Wellington

Surveyor General: Warren N. Hawkey

Ministry of Works and Development

Private Bag

Wellington

Commissioner: R. G. Norman

P.O. Box 12346

Wellington

Director: Dr. D. Hurley

New Zealand Mines Department

\section{NICARAGUA}

(No recent information)

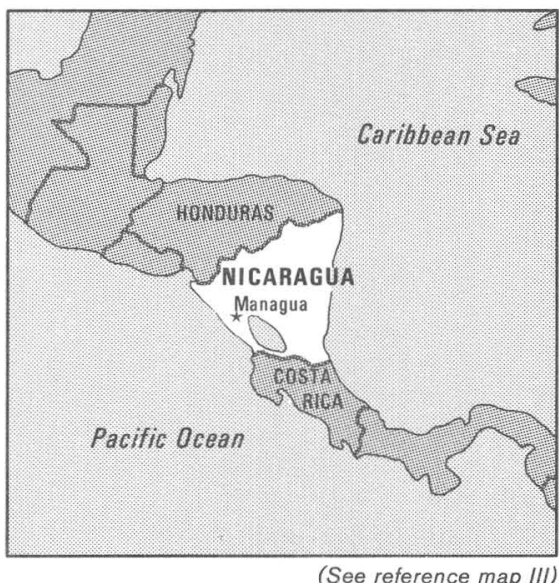

G

Proyecto Geotérmico

Empresa Nacional de Luz y Fuerza (ENILUF)

Apartado 55

Managua

Director: Emilio Rappaccioli

Corporación Nicaraguense de Minas e Hidrocarburos (CONDEMIA)

Director: Dr. R. P. Suggate

New Zealand Oceanographic Institute, Evans Bay

Oil and Gas Exploration

P.O. Box 6342

Te Aro, Wellington

Chief Mines Engineer (Minerals): A. B. Cowie
Apartado Postal No. 8

Managua, D.N.

Ministro: Carlos Zarruck

G

G

Instituto Nicaraguense de Recursos Naturales y del Ambiente (IRENA)

Apartado Postal No. 5123

Managua, D.N.

Director: Jorge Jenkins

Instituto de Investigación Sísmica

Apartado Postal No. 1761

Managua, D.N.

C

Instituto Geográfico Nacional

Ministerio de Fomento y Obras Publicas

Apartado Postal 2120

Managua, D.N.

Director: Adolfo Espino G. 


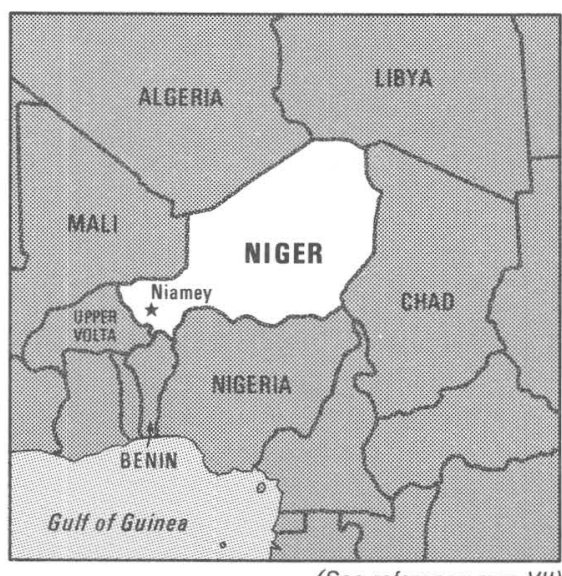

(See reference map VII)

G,H Direction des Mines et de la Géologie

Ministère des Mines et de l'Hydraulique

BlP. 257

Niamey

Secretary General: Sori Boubacar

Directeur of Mines: Issoufou Mahamane

Service Hydrologique de Génie Rural

Ministère du Developpement Rural

B.P. 241

Niamey

Directeur: Ali Seyni

Direction du Service Topographique et du Cadastre

Ministère des Finances

B.P. 250

Niamey

Directeur: Abdou Kané

H Office des Eaux de Sous-sol (OFEDES)

B.P. 734

Niamey

Directeur: Imini Mahamane

Office Nationale des Recherches Minieres (ONAREM)

B.P. 210

Niamey

Directeur: Sani Koutoubi

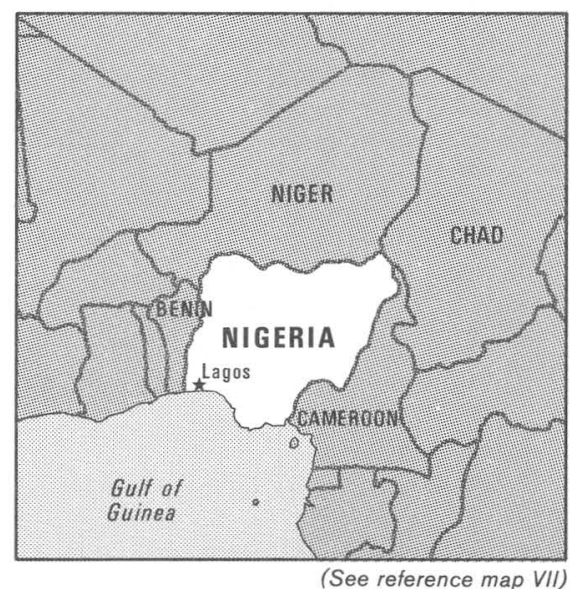

G,H Geological Survey Department of Nigeria Ministry of Mines and Power

P.M.B. 2007

Kaduna South, Kaduna State

Director: C. N. Okezie

G

Ministry of Mines and Power

Lagos Office, Geologial Survey Department

P.M.B. 12574

Lagos

Principal Geologist: H. T. Molokwu

G

Nigerian Coal Corporation

P.M.B. 1053

29 Okpara Ave

Enugu, Anambra

Administrator: O. A. Udemah

G

Nigerian Mining Corporation

24 Naraguta Ave.

P.M.B. 2154

Jos, Plateau State

General Manager: Alhaji Rilwanu Lukman

G

Ministry of Mines and Power

Lagos Office, Mines Division

P.M.B. 12574

Lagos

Director of Mineral Resources:

Alhaji Inua Gombe

$\mathbf{R}$
Nigerian National Petroleum Corporation P.M.B. 12701

Lagos

Managing Director: F. R. A. Marinho 
Geological Survey Department

P.M.B. 2007

Kaduna South, Kaduna State

Officer in Charge: J. A. Ogunkoya

C

Surveys Division

Federal Ministry of Works

Igbosere Road at Okesuna Street

Lagos

Director of Federal Surveys:
A. O. Adebekun

H

Federal Ministry of Water Resources

9 Kofo Abayomi Street

Victoria Island

P.M.B. 12700

Lagos

Minister: Dr. Bykar Shaib

R Federal Ministry of Mines and Power

Broad Street Office

Lagos Office, Geological Survey Department

Lagos

Minister: Alhaji Rilwanee Lukman

\section{NORWAY}

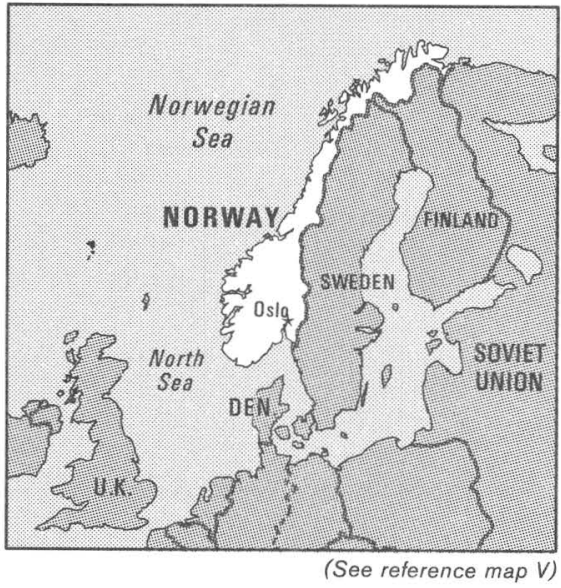

G

Norges Geologiske Unders $\varnothing$ kelse

[Norway Geological Survey]

Leiv Erikssons Vei 39

P.B. 300

7001 Trondheim

Managing Director: Knut S. Heier

G
G,C
Norwegian Geotechnical Institute

P.O. Box 40 Tasen

Oslo

Director: Dr. Kaare Hoeg

Norsk Polarinstitutt

[Norwegian Institute for Polar Affairs]

Rolfstangveien 12

Postboks 158

1330 Oslo Lufthavn

Director: Tore Gjelsvik

Continental Shelf, Institute (IKU)

Hakon Magnussonsgt. 1B

Postboks 1883

7001 Trondheim

Director: Tore Hartvig

Norsk Institutt for Vannforskning

[Norwegian Institute for Water Research]

Postboks 333, Blindern

Oslo 3

Chief: Lars Overrein

Ministry of Environment

Myntgata 2

Postboks 8013 DEP

Oslo 1

Director General for Resources: Kjell Glomnes

Oljedirektoratet

[Norwegian Petroleum Directorate]

Laagaardsveien 80

4000 Stavanger

Director: Fredrik Hagemann

Ministry of Petroleum and Energy

Tollbugata 31

Oslo 1

Director-General: Knut Daehlin 


\section{OMAN}

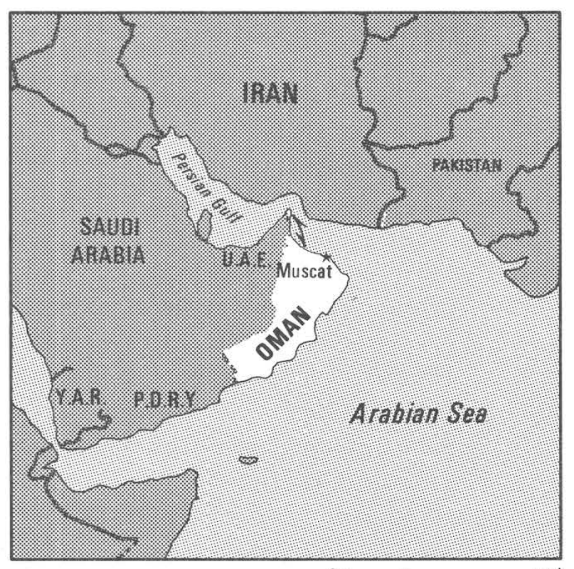

(See reference map VI)

G, R Ministry of Petroleum and Minerals

P.O. Box 551

Muscat

Minister: Said Ahmad Al-Shanfari

Director General of Technical Affairs: Khalifa Mubarak Al-Hinai

Ministry of Agriculture and Fisheries

P.O. Box 467

Muscat

Minister: Abdul Hafez Salim Rajab

C

Ministry of Defence

P.O. Box 113

Muscat

Deputy Prime Minister for National Security and Defense: Sayyid Fahar bin Taimur al-Said

Ministry of Communications

P.O. Box 684

Muscat

Contact:. Sayyid Salim Bin Nasser

Al-Busaidi

Public Authority for Water Resources

P.O. Box 5225

Ruwi

Chairman: Abdul Hafiz Salim Rajab

Technical Secretary: Dr. Robert Dale

\section{PAKISTAN}

(No recent information)

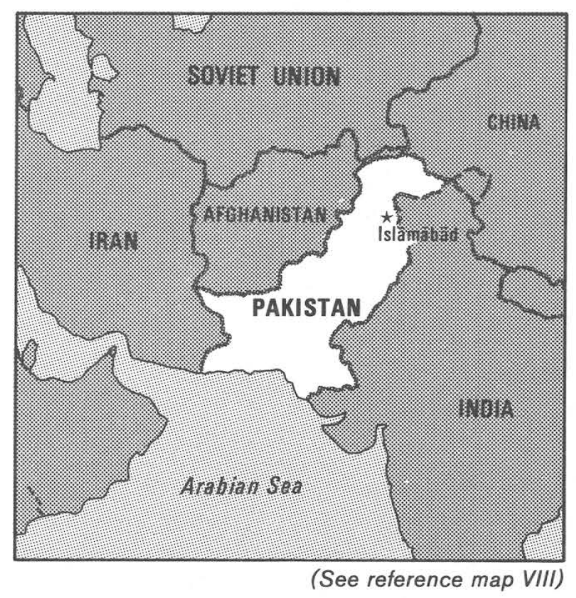

G

G

G

Resource Development Corporation 8th Floor, Dawood Center

Karachi-4

General Manager: S. A. Bilgrami

Oil and Gas Development Corporation

Shafi Chambers, Club Road

Karachi-4

Chairman: Iftikharuddin Ahmed

Geological Survey of Pakistan

P. O. Box 15

Quetta

Director General: Asrarullah

C

Survey of Pakistan

Shahrah-i-Muhammad Raza Shah Pehlvi

Post Box 10

Rawalpindi

Surveyor General: Mian Muhammad Sharif

Water and Power Development Authority

WAPDA House

Lahore

Chairman: Fazle Raziq

$\mathrm{H}$

Ministry of Water and Power

Secretariat Block "A"

Islamabad

Additional Secretary: M. Q. Siddiqui

$\mathrm{H}$
Flood Forecasting and Warning Center

46 Gulberg Road

Lahore

Member: Zarif Ahmed 
Ministry of Petroleum and Natural Resources

Secretariat Block "A"

Islamabad

Secretary, Petroleum Division: T. H. Hashmi

Secretary, Water, Power and Natural Resources Division: Masroor Hasan Khan

R

Pakistan Minerals Development Corporation (PMDC)

PIDC House

Dr. Ziauddin Ahmed Road

Karachi

Chairman: F. K. Khilji

\section{PANAMA}

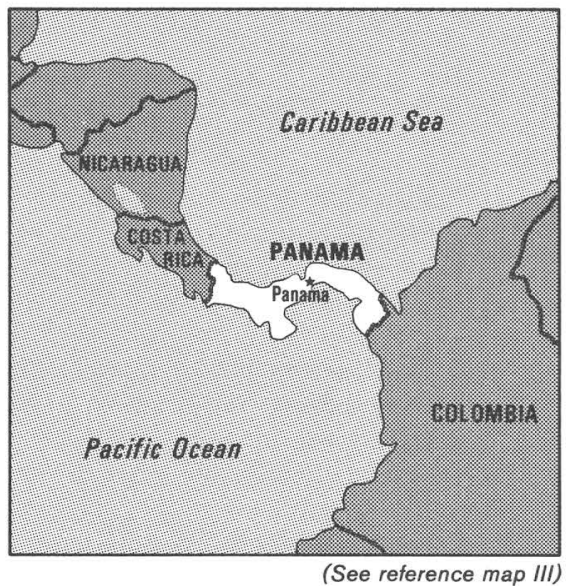

G, R

Dirección General de Recursos Minerales

Apartado Postal 9658, Zona 4

Panama

Director: Julio Mérida

G, R Dirección General de Hidrocarburos

Apartado Postal 9658, Zona 4

Panama

Director: Eng. Nitzia de Villarreal

G

Corporación de Desarrollo Minero-Cerro Colorado (CODEMIN)

Apartado 5312

Panama 5

Presidente: Eng. Jaime Roquebert

C Instituto Geográfico Nacional "Tommy Guardia"

Apartado Postal 5267, Zona 5

Panama

National Director: José Antonio Tejada
Instituto de Acueductos y Alcantarillados Nacionales (IDAAN)

Apartado 5234

Panama 5

Director: Ivan Estribi

$\mathrm{H}, \mathrm{G}$ Instituto de Recursos Hidráulicos y Electrificación (IRHE)

Apartado 5285

Panama 5

Director:. Edwin Fabrega

H,R Dirección Nacional de Recursos Nacionales Renovables (RENARE)

Ministerio de Desarrollo Agropecuario

Apartado 2016

Paraiso, Corregimiento de Ancón

Director: Dr. Sergio Castillo Martinez

\section{PAPUA NEW GUINEA}

(No recent information)

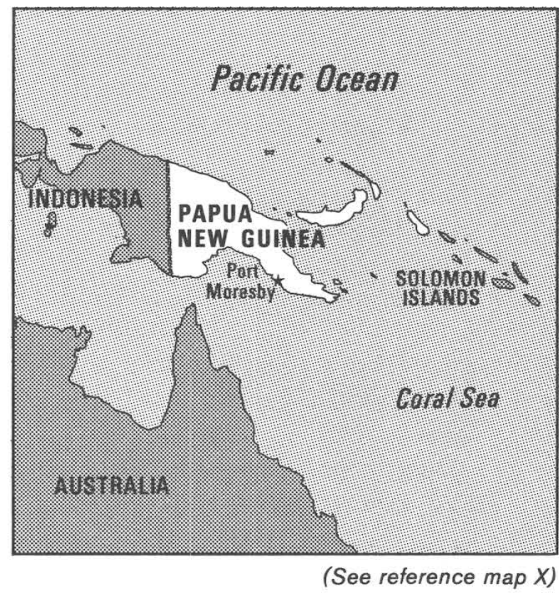

G Geological Survey Division Office of Minerals and Energy Box 778

Port Moresby

Chief Geologist: A. McKinlay

Department of Natural Resources

Papua

Surveyor General: Kisokau Pochapon 


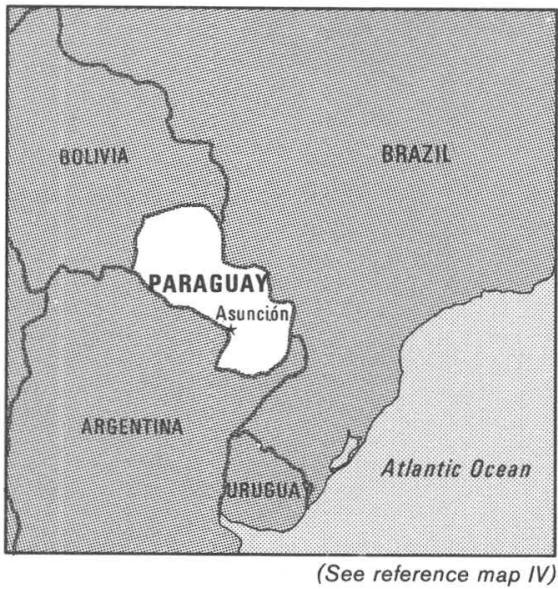

C

Instituto Geográfico Militar

Avenida Artigas casi Perú

Asunción

Director: Gral. Abraham Abed

G,R Dirección de Recursos Minerales

Ministerio de Obras Pûblicas y Comunicaciones

Calle Alberdi y Oliva

Asunción

Director: Ing. Luis Alberto Ruotti

G

Institute of Basic Sciences

Ciudad Universitaria

Km 10, San Lorenzo

Director: Prof. Dr. Narciso Gonzalez R.

Department of Science and Technology

Ciudad Universitaria, Lambare

Director: Dr. Luis Meyer

Dirección de Meteorología

Ministerio de Defensa Nacional

Av. Mariscal López y Vice Presidente Sánchez

Asunción

Director: Lic. Francisco Solano Santacruz

Administración Nacional de Navegación y Pertos Plazoleta Isabel La Católica

Asunción

Presidente: Contralmirante Ramón E. Martino

R
Dirección de Industrias Militares

Ministerio de Defensa Nacional

Avenida General Santos y Manuel Domínguez

Asunción

Director: Gral. Robert P. Knopfelmacher

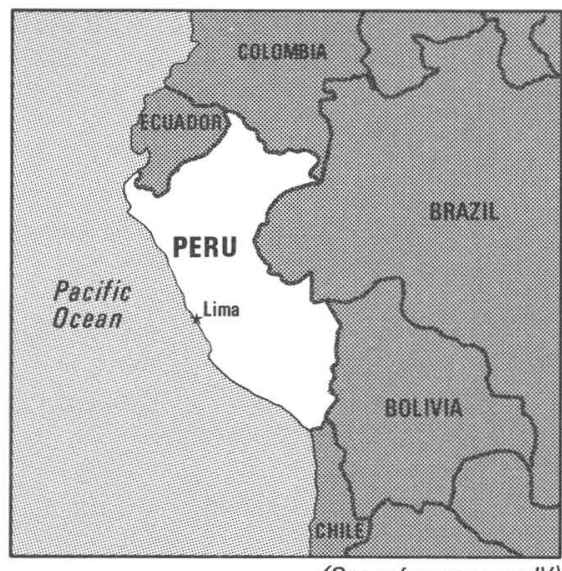

(See reference map IV)

G

G

G,C

G

G

G,C,H,R Oficina Nacional de Evaluación de Recursos Naturales (ONERN)

Calle 17 \# 355, Urb. El Palomar, San Isidro

Lima

Jefe Encargado: Carlos Zamora 
Servicio Aerofotográfico Nacional (SAN)

Base Aérea "Las Palmas"

Barranco, Lima

Director: Mayor Gral. F. A. P. Oscar Carrera

C

Instituto Geográfico Militar

Av. Andres Aramburu 1198

Apartado 2038

Lima

Director General: General E. P. Alberto Delgado $\mathrm{V}$.

$\mathrm{C}, \mathrm{H}$ Dirección de Hidrografía y Navegación de la Marina (DHNM)

Calle Saenz Peña No. 590, La Punta (CAllao)

Casilla Postal \#80, Callao

Lima

Director: Contralmirante A. P. Jorge del Aguila

$\mathrm{H}$

Servicio Nacional de Meteorología e Hidrología (SENAMHI)

Rep. de Chile 295, Of. 205

Lima

Director: General F. A. P. Jacobo Delmar

$\mathbf{R}$

R

R
Dirección General de Hidrocarburos

Ministerio de Energia y Minas

Edificio Sol Gas

Esq. Av. Javier Prado y Aviación

Urb. San Borja, Lima

Director: Luis del Castillo

Petroleos del Perú (PETROPERU)

Paseo de la Republica No. 3361

San Isidro, Lima

Presidente: Juan F. Cassabonne

Dirección General de Minería

Ministerio de Energia y Minas

Urb. San Borja, Lima

Director General: Luis Gonzales Cacho

Minister of Energy and Mines:

Juan Inchaustegui Vargas
PHILIPPINES, THE

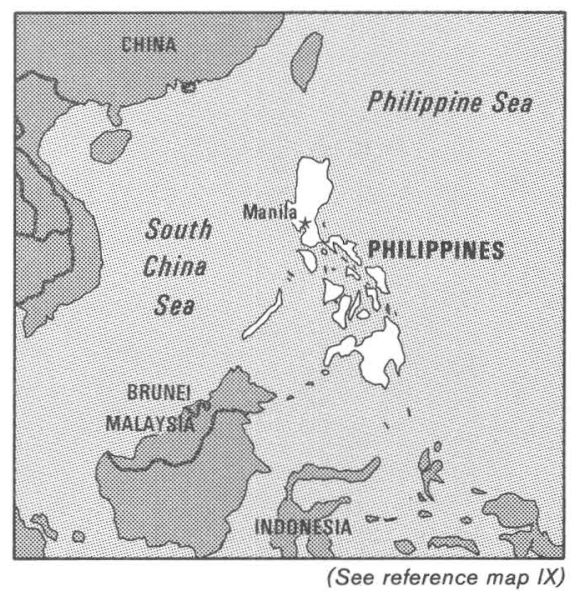

Philippine Institute of Volcanology

National Science and Technology Authority

Hizon Building, Quezon Avenue

Quezon City

Director: R. S. Punongbayan

G

G

G

G

G

G,R Bureau of Mines and Geosciences

P.O. Box 1595

Pedro Gil Street

Manila 2801

Director: J. C. Fernandez

Chief, Geological Survey Division: Dr. Guillermo Balse 
National Power Corporation

NPC Complex

Quezon Blvd. Extension

Corner BIR Road, East Triangle

Diliman, Quezon City

President:. G. Y. Itchon

Ministry of Public Works and Highways

Bonifacio Drive

Minister: Jesus Hipolito

National Irrigation Administration

National Irrigation Administration Building

Epifanio Delos Santos Avenue

Quezon City, Metro Manila

Administrator:. Cesar L. Tech

$\mathrm{H}$

National Water Resources Council

National Irrigation Administration Building

Epifanio Delos Santos Avenue

Quezon City 3008

Executive Director: Angel A. Alejandrino

C

Bureau of Coast and Geodetic Survey

421 Barraca Street

Binondo, Manila 2807

Director: A. P. Ventura

\section{POLAND}

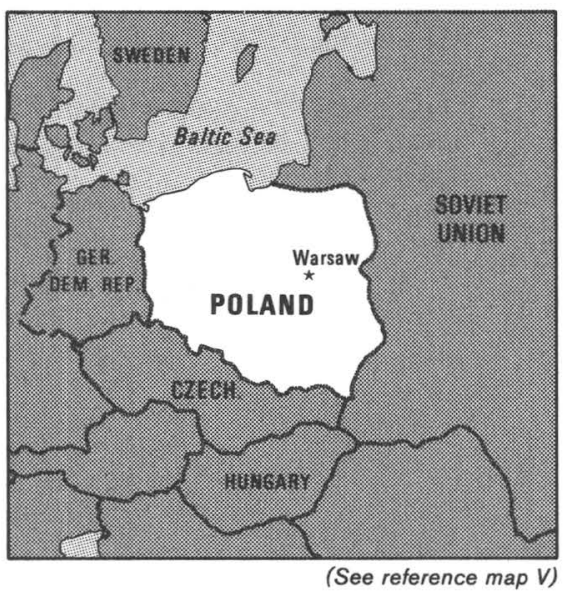

G

Zaklad Paleobiologii PAN

A1. Zwirki i Wigury 93

02-089 Warszawa

Director: Prof. Halszka Osmolska

G

Instytut Geofizyki PAN
Port Area, Manila

G

G,R

G

G

G

G,H,C,R

C

C

C

ul. Pasteura 3

02-093 Warszawa

Director: Prof. Jerzy Jankowski
Glówny Instytut Górnictwa

[Central Mining Institute]

Plac Gwarków I

40-951 Katowice

Director: Dr. Jozef Pazdziora

Centralny Urzad Geologii

[Coordinative and Administrative Body]

ul. Jasna 6

00-013 Warszawa

President: Dr. Zdziaslaw Dembowski

Instytut Górnictwa Naftowego i Gazownictwa [Institute of Petroleum]

ul. Lubicz 25a

31-503 Kraków

Director: Prof. Józef Raczkowski

Instytut Nauk Geologicznych PAN

Al. Zwirki i Wigury 93

02-089 Warszawa

Director: Prof. Maria Borkowska

Zaklad Oceanologii PAN

ul. Powstancow Warszawy 55

81-967 Sopot

Director: Prof. Czeslaw Druet

Muzeum Ziemi PAN

Al. Na Skarpie 20/26

00-488 Warszawa

Director: Dr. Krzysztof Jakubowski

Instytut Geologiczny

ul. Rakowiecka 4

00-975 Warszawa

Director: Prof. Waclaw Ryka

Instytut Meteorologii i Gospodarki Wodnej

[Institute of Meteorology and Water

Management]

ul. Podlésna 61

01-673 Warszawa

Director: Dr. Maria Cetnarowicz

Glówny Urzad Geodezji i Kartografii

[Coordinative and Administrative Body]

ul. Jasna $2 / 4$

00-013 Warszawa

President: Prof. Zdzislaw Adamczewski

Instytut Geodezji i Kartografii

ul. Jasna $2 / 4$

00-013 Warszawa

Director: Prof. Bogdan Ney

Instytut Geografii i

Przestrzennego

Zagospodarowania PAN

ul. Krakowskie Przedmiescie 30

00-325 Warszawa

Director: Prof. Jerzy Kastrowicki 


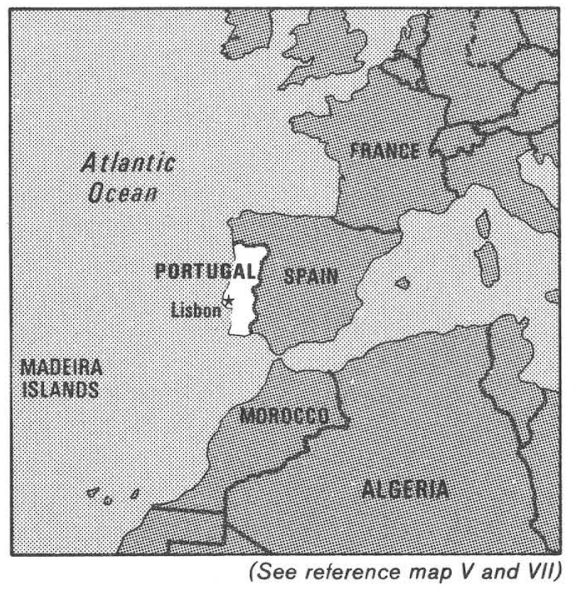

G

G

G,H Laboratório Nacional de Engenharia Civil Ministério de Equipamento Social Avenida do Brasil, 101 1799 Lisboa Codex Director: Eng. Júlio Ferry Borges

Direccâo-Geral de Geologia e Minas Ministerio da Indústria e Enérgia Rua António Enes, 5 1000 Lisboa

Director: Dr. Alcides Rodrigues Pereira

Serviços Geológicos de Portugal Rua da Academia de Ciências, 19-2 ${ }^{\circ}$ 1200 Lisboa

Director: Dr. Delfim de Carvalho

Instituto Geográfico e Cadastral Ministério das Finances e do Plano 1200 Lisboa

Director-Geral: Eng. Rui Henriques Galeano Barata Pinto

Direccão-Geral dos Recursos e Aproveitamentos Hidráulicos Ministério do Equipamento Social Rua de S. Mamede, 23 1100 Lisboa Director-Geral: Eng. Adolfo Goncalves

Portuguese Commission on International Hydrologic Program

Ministério do Equipamento Social

Rua de S. Mamedo, 23

1100 Lisboa

President: Eng. Adolfo Goncalves

Ministério da Qualidade de Vida

Rua Prof. Gomas Teixeira

1300 Lisboa

Minister: Dr. António d'Orey Capucho

Ministério da Indústria e Energia

Rua da Horta Seca, 14

1200 Lisboa

Minister: Prof. Dr. José Veiga Simão Praça da Estrela

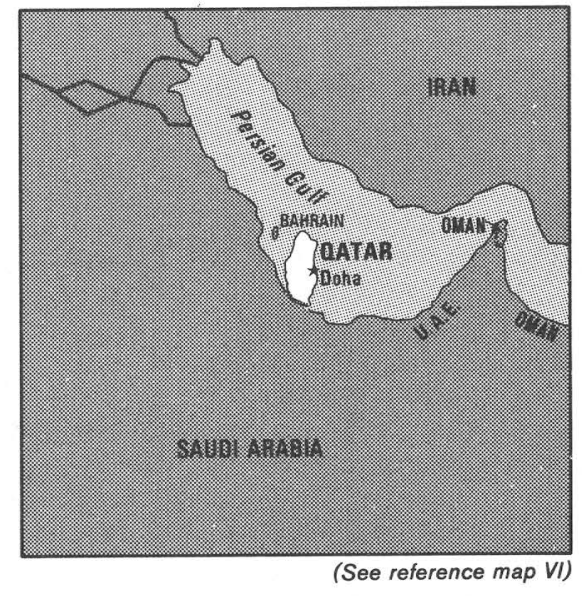

G,H,C Industrial Development Technical Center (IDTC)

P.O. Box 2599

Doha

Director: Said Mishal

H

Water Resources Section Integrated Water and Land Use Project P.O. Box 1967

Doha

Chief:. Mohamed Abdul Wahab

H

Food and Agriculture Organization United Nations Development Program P.O. Box 3237

Doha

Chief Technical Advisor: John Pike

R, H,C Department of Petroleum Affairs Ministry of Finance and Petroleum P.O. Box 83

Doha

Director: Abdullah Sallat 


\section{REUNION}

(No recent information)

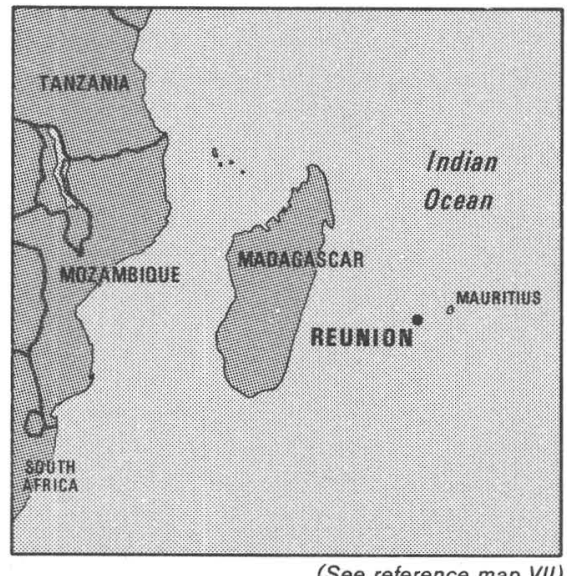

G

Services des Travaux Publics

St. Denis

\section{ROMANIA}

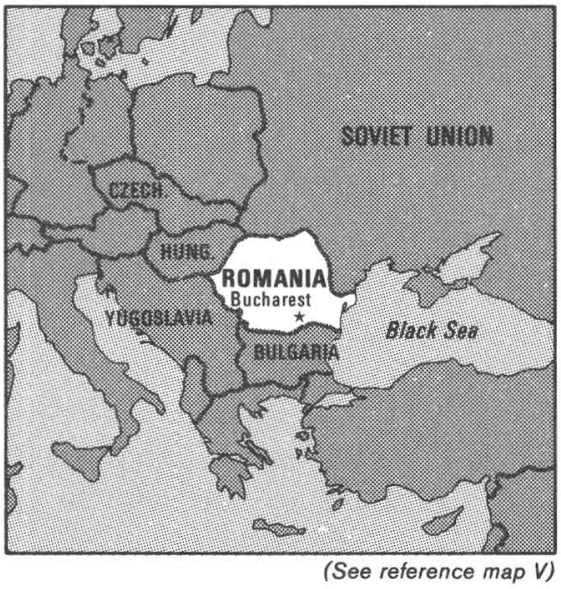

G

Ministerul Geologiei

Str. Mendeleev no. 36-38

Bucharest

Minister: Ioan Folea

G

Institutul de Geologie si Geofizicå

Str. Caransebes no. 1

Sector 7, Bucharest

Director: Iosif Bercia

G
Comisia Romania pentru Activitati Spatiale [Remote Sensing]

Str. Roma 32-34

Bucharest

President: Constantin Teodorescu

G,C Institutul de Geografie si Geologie

Str. Dimitrie Racovită no. 9

Bucharest

Director Professor: Gratian Ceoflică

Scientific Director: Petre Ghistescu

H Institutul de Meteorologie si Hidrologie

Sos, Bucuresti-Ploiesti

Bucharest

Director: Constantin Diaconu

$\mathbf{R}$

Ministerul Minelor

Str. Mendeleev no. 36-38

Bucharest

Minister: Ion Lazarescu

$\mathbf{R}$

Ministerul Petrolului

Calea Victoriei no. 109

Bucharest

Minister: Gheorghe Vlad

\section{RWANDA}

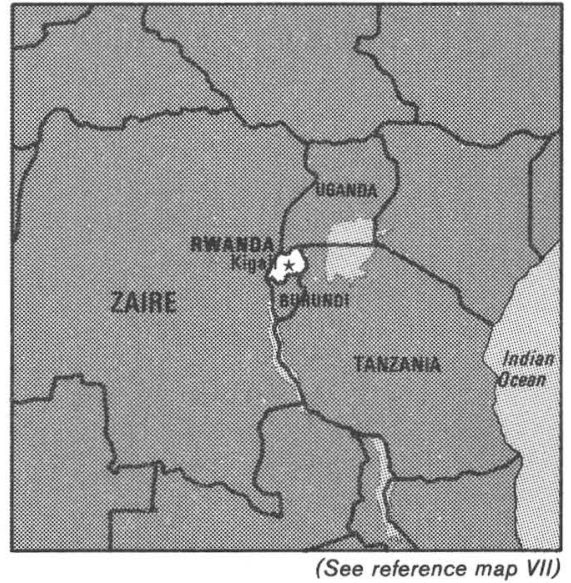

G,R,C Ministère des Ressources Naturelles

B.P. 413

Kigali

Minister: M. D. Iyamuremye

Director General, Service des Ressources Naturelles: Casimir Karangwa

Director: Division de la Cartographie: T. Bizimungu

Director General, Service des Mines et Carrières: P. Mahungiro

Director, Mining Research: I. Kifnir 


\section{SAUDI ARABIA}

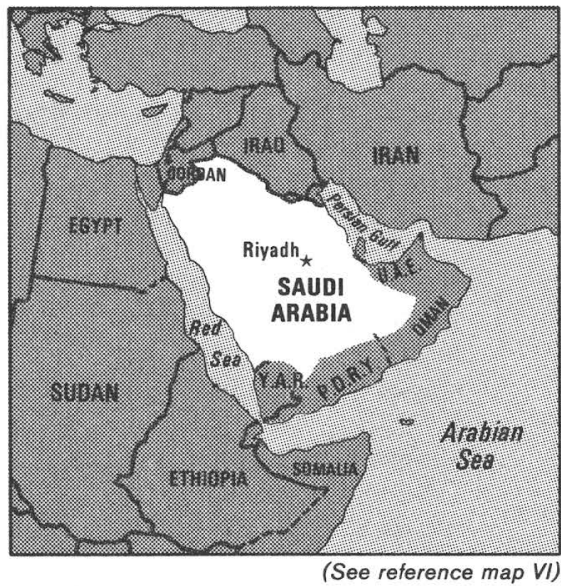

G, R Ministry of Petroleum and Mineral Resources Deputy Ministry for Mineral Resources P.O. Box 2880

Jiddah

Deputy Minister for Mineral Resources: Ghazi Sultan

G

University of Petroleum and Minerals

Department of Earth Science

P.O. Box 144, Dhahran International Airport ATTN: Box 287, UPM

Dhahran

Chairman: Adnan M. Miazy

G,H University of Petroleum and Minerals P.O. Box 144, Dhahran International Airport ATTN: Box 274, UPM

Dhahran

Rector of the University: Dr. Bakr A. Bakr

G

King Abdul Aziz University

Faculty of Earth Sciences

P.O. Box 1450

Jiddah

Dean: Dr. Abdul Razák Bakr

C Ministry of Petroleum and Mineral Resources Aerial Survey Department

P.O. Box 247

Riyadh

Director General: Soliman Saleh Al-Robaishy

Saline Water Conservation Corporation P.,O. Box 5968

Riyadh

Governor: Dr. Abdullah Mohammad Al-Ghulaiqa

Ministry of Agriculture and Water

Water Resources Development Department P.O. Box 478

Riyadh

Director: Mustafa Noory

R

Ministry of Petroleum and Mineral Resources P.O. Box 247

Riyadh

Minister: Ahmad Zaki Yamani

\section{SENEGAL}

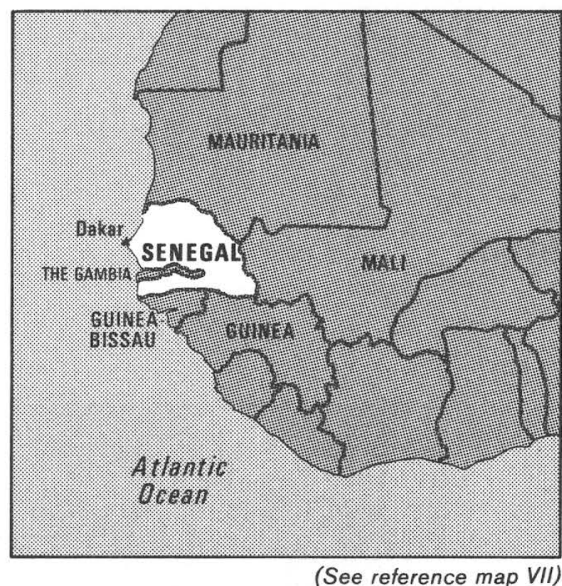

Direction des Mines et de la Géologie Ministère du Developpement Industrial Route de Ouakam

B.P. 1238

Dakar

Director: Babacar Faye

Office de la Recherche Scientifique et Technique d'Outre-Mer (ORSTOM) ${ }^{9}$

Route des Peres Maristes-Hann

B.P. 1386

Dakar

Director: Bernard Dalmayrac

G

Centre de Géophysique

Office de la Recherche Scientifique et Technique d'Outre-Mer (ORSTOM) ${ }^{9}$

B.P. 50

M'Bour

Director: Bernard Dalmayrac

\footnotetext{
${ }^{9}$ French research organization; no governmental affiliation
} 
Bureau de Recherches Géologiques et Minières $\left(\right.$ BRGM) ${ }^{9}$

7, rue Mermoz

B.P. 268

Dakar

Director: Remy Delafosse

C Institut Géographique National (IGN)

7, rue Mermoz

B.P. 4016

Dakar

Director: Gilbert Durieux

C Service Géographique du Senegal

14 , rue Victor Hugo

B.P. 740

Dakar

Director: Serigne M'Baye Thiam

Direction de l'Hydraulique Urbaine et Rurale Ministère de l'Equipement

B.P. 2014

Route des Peres Maristes

Dakar

Director: Bocar Cisse

Institut Fondamental d'Afrique Noire (IFAN) Universite de Dakar

B. P. 206

Dakar

Director: Amar Samb

Centre d'Etudes et de Recherches sur les Energies Renouvelables (CERER) - Hann

Secretariat d'Etat a la Recherche Scientifique et Technique

B. P. 476

Dakar

Director: Djibril Fall

Direction de la Meteorologie Nationale (DMN)

Ministere de l'Equipement

Aeroport de Dakar-Yoff

B.P. 8257

Dakar

Director: Dr. Mansour Seck

R

\section{PETROSEN}

25, Avenue Georges Pompidou

B. P. 2076

Dakar

Director: Samba Diouf

\section{SIERRA LEONE}

(No recent information)

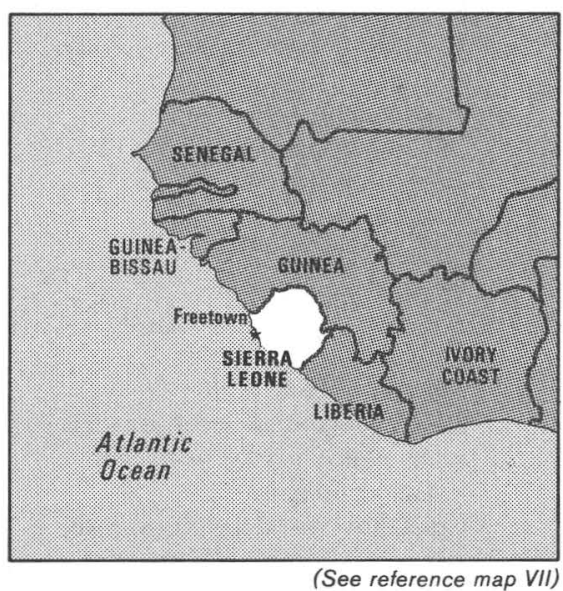

G

H

Geological Survey Division

Ministry of Lands, Mines, and Labor

New England, Freetown

Director: A. H. Gabiri

Water Resources Division

Ministry of Energy and Power

Leone Building, Siaka Stevens Street

Freetown

Chief Engineer: I. S. Kabia

C Surveys and Land Division

Ministry of Lands and Mines, and Labor

New England, Freetown

Director: E. A. Redwood-Sawyer

\section{SINGAPORE}

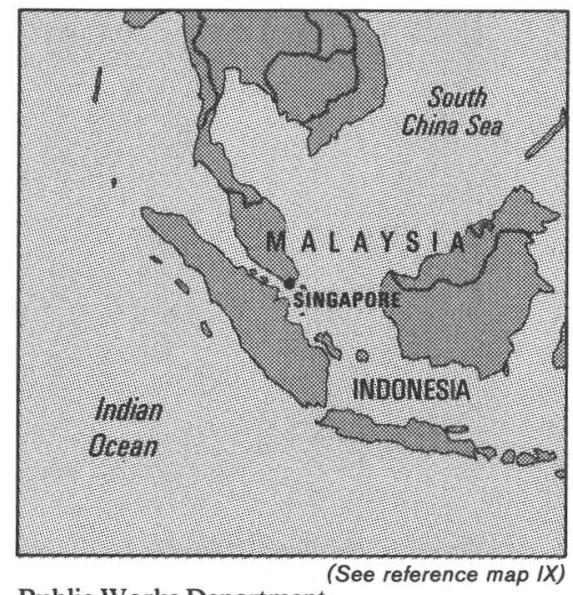

Public Works Department

Structural Design and Investigation Branch

Ministry of National Development

10th Floor National Development Building

Maxwell Road

Singapore 0106

Director of Public Works: Yap Neng Chew 


\begin{tabular}{|c|c|}
\hline $\mathrm{G}, \mathrm{H}$ & $\begin{array}{l}\text { Science Center } \\
\text { Science Center Building } \\
\text { Science Center Road } \\
\text { Singapore } 2260 \\
\text { Scientific Officer (Physical Sciences): Tey Mui Lee }\end{array}$ \\
\hline C & $\begin{array}{l}\text { Head Mapping Unit } \\
\text { Ministry of Defense } \\
\text { Dover Road Camp I } \\
\text { Dover Crescent } \\
\text { Singapore } 0513 \\
\text { Head: Yuen See Wah }\end{array}$ \\
\hline C & $\begin{array}{l}\text { Land Surveyors Board } \\
\text { Ministry of Law } \\
\text { 3d Floor, National Development Building } \\
\text { Maxwell Road } \\
\text { singapore } 0106 \\
\text { Chief Surveyor: Kwah Kheng Swee }\end{array}$ \\
\hline $\mathbf{H}$ & $\begin{array}{l}\text { Public Utilities Board } \\
\text { Water Department } \\
\text { PUB Building } \\
\text { Somerset Road } \\
\text { Singapore } 0923 \\
\text { Chief Water Engineer: Owen Liu (Ag) }\end{array}$ \\
\hline $\mathbf{R}$ & $\begin{array}{l}\text { Economic Development Board } \\
\text { 9th Floor, World Trade Center } \\
\text { Telok Blangah Road } \\
\text { Singapore } 0409 \\
\text { Chairman: P. V. Hwang }\end{array}$ \\
\hline
\end{tabular}

\section{SOLOMON ISLANDS}

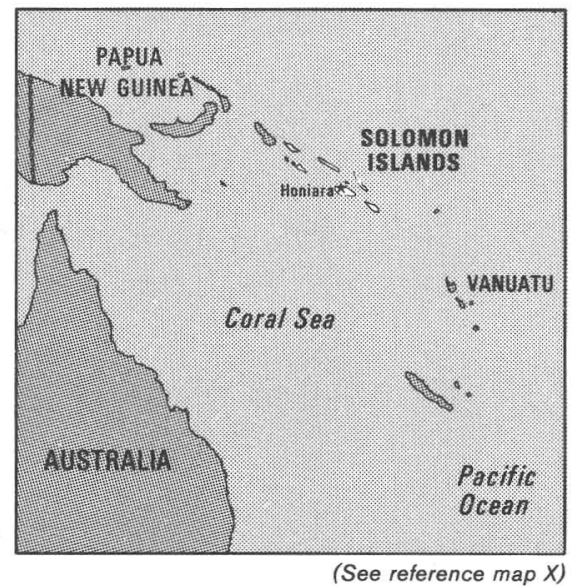

G

Ministry of Natural Resources
G,R Geology Division

Ministry of Lands, Energy and Natural Resources Honiara, Guadalcanai

Chief Geologist: S. Danitofea

\section{SOMALIA}

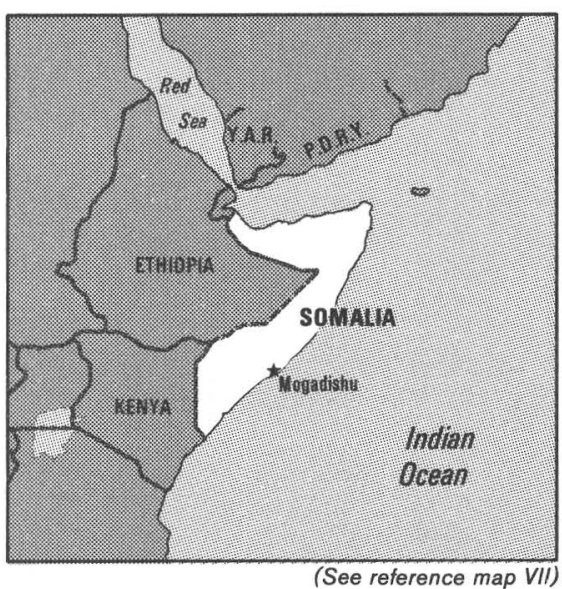

$\mathrm{G}, \mathrm{H}$

Geological Survey Department

Ministry of Minerals and Water Resources P.O. Box 744

Mogadishu

Director: Mohamed Said Abdi

Hydrogeological Department Ministry of Minerals and Water Resources P.O. Box 744

Mogadishu

Director: Mohamed Yusuf Awale

Hydrogeological Department

Water Development Agency

P.O. Box 525

Mogadishu

General Manager: Khalif Haji Farah

Hydrology Department

Ministry of Agriculture

Mogadishu

Director: Ibrahim Muse

Survey and Mapping Department

Ministry of Defense

P.O. Box 24

Mogadishu

Chief: Colonel Ibrahim Elmi Gelle

Geology Department

P.O. Box G24

Honiara, Guadalcanal

Chief Geologist: B. Hackman 


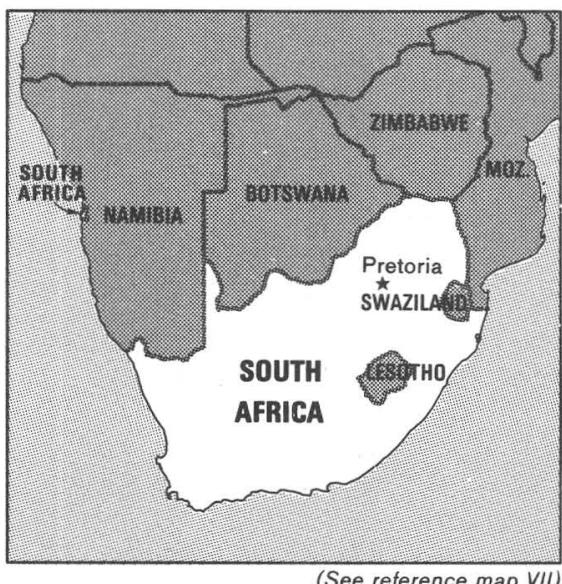

G

$\mathrm{G}, \mathrm{H}$

C

(Geomagnetism, Seismology)

Council for Scientific Research (CSIR)

P.O. Box 32

Hermanus, Cape Province

7200

Director: Dr. G. J. Kuhn

Geological Survey of Southern Africa

223 Visagie Street

Pretoria

0002

(Private Bag X112, Pretoria, 00001)

Director: L. N. J. Engelbrecht

Director Surveys and Mapping

Private Bag

Mowbray, Cape Town

7700

Chief Director: E. Van der Spuy

$\mathrm{H}$

National Institute for Water Research

Council for Scientific and Industrial Research (CSIR)

P.O. Box 395

Pretoria

0001

Chief Director: Dr. G. G. Cilliers

H
Minerals Bureau

Queensbridge Buildings

60 Juta Street

(Private Bag X4)

Braamfontein

2017

Chief Director: Dr. P. J. Hugo

R

R

$\mathrm{H}$ Commerce and Tourism

Legal and General Building

Cor. Pretorius and Prinsloo Streets

(Private Bag X84)

Pretoria

0001

Minister: Dr. D. J. De Villiers

Department of Mineral and Energy Affairs P.O. Box 616

Pretoria

0001

Minister: P. T. C. Du Plessis

Bloemfontein University

Institute for Ground Water Studies

P.O. Box 339

Bloemfontein

9300

Director: Prof. F. D. I. Hodgson

G

Grahamstown University

P.O. Box 94

Grahamstown

6140

Head of Geography: Dr., Andrew Stone

G

Natal University

P.O. Box 375

Pietermaritzburg

3205

Head of Geology: Prof. R. E. Schulze

Head of Geography: Prof. J. Hank 


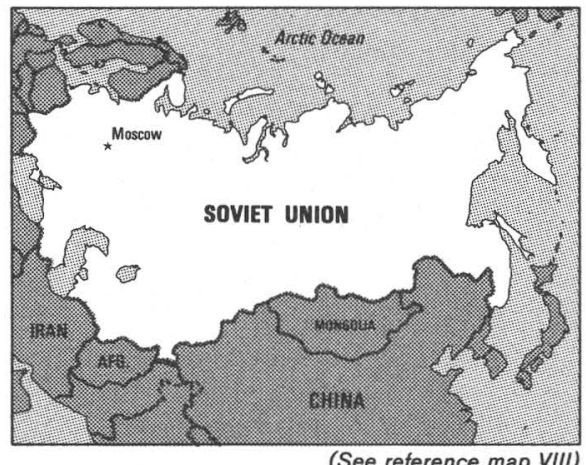

G

G

G

G

G

G
Geology Scientific Research Institute [All-Union] (VSEGEI)

Sredniy Prospekt 71

199026 Leningrad

Director: A. I. Zhamoyda

Institute of Geology

Akademiya Nauk USSR

109017 Moscow ZH-17

Pyzhevskiy Pereulok 7

Director: P. P. Timofeyev

Institute of Geochemistry and Analytical

Chemistry imeni V.I. Vernadskovo

Akademiya Nauk USSR

117975 GSP-1 Moscow V-334

Vorob'yevskoye Shosse 47-A

Director: V. L. Barsukov

Institute of Earth Physics imeni O. Yu. Shmidta

Akademiya Nauk USSR

123810 Moscow D-242

Bolskaya Gruzinskaya Ulitsa 10

Director: M. A. Sadovskiy

Department of Geology, Geophysics, and Geochemistry

Akademiya Nauk USSR

117901 GSP-I Moscow V-71

Leninskiy Prospekt 14

Academician Secretary: B. S. Sokolov

Institute of the Geology of Ore Deposits, Petrography, Mineralogy and Geochemistry, Akademiya Nauk USSR

109017 Moscow ZH-17

Staromonetniy Pereulok 35

Director: F. V. Chukhrov

Institute of Lithosphere

Afkademiya Nauk USSR

109180 Moscow ZH-180

Staromonetniy Pereulok 22

Director: N. A. Bogdanov
Mineralogical Museum imeni A. E. Fersmana Akademiya Nauk USSR

117071 Moscow V-71

Leninskiy Prospekt 18

G

G

G
Department of Oceanology, Physics of the Atmosphere and Geography

Akademiya Nauk USSR

117901 Moscow V-71

Leninskiy Prospekt 14

Academic Secretary: L. M. Brekhovskikh

Institute of Oceanology imeni P. P. Shirshova Akademiya Nauk USSR

117218 Moscow V-218

Ulitsa Krasikova 23

Director: A. S. Monin

Institute of Vulcanology

Far East Scientific Center

Akademiya Nauk USSR

683603 Petropavlovsk

Kamchatskiy, Bul'var Piypa 9

Director: S. A. Fedotov

Institute of Geology and Geophysics of the Siberian Department

Akademiya Nauk USSR

630090 Novosibirsk 90

Universitetskiy Prospekt 3

Director: A. A. Trofimuk

Institute of Permafrost Studies

Siberian Department

Akademiya Nauk USSR

677010 Yakutsk 10

Director: P. I. Melnikov

Institute of the Earth's Crust

Siberian Department

Akademiya Nauk

664033 Irkutsk 33

Ulitsa Lermontova 128

Favors Kovo Ulitsa 1

Director: N. A. Logachev

Ministry of Geology of the USSR

Moscow 123242

Bolshaya Gruzinskaya, Ulitsa 4/6

Minister: Ye. A. Kozlovskiy

State Committee for Hydrometeorology and Environmental Control

Akademiya Nauk USSR

Pereulok Pavlika Morozova 12

Moscow D-376

Director: Y. A. Ivanov 
Institute of Hydrodynamics imeni M. A.

Lavrent'yev

Siberian Department

Akademiya Nauk USSR

630090 Novosibirsk 90

Prospekt Nauki 15

Director: L. V. Ovsyannikov

Institute of Water Problems

Akademiya Nauk USSR

103064 Moscow K-64

Sadovaya Chernogryazckaya $13 / 3$

Director: G. V. Voropayev

Main Administration for Geodesy and

Cartography (GUCK)

Applied Geodesy SRI, Novosibirsk

NII Prikladnoy Geodezii

Director: I. T. Antipov

\section{SPAIN}

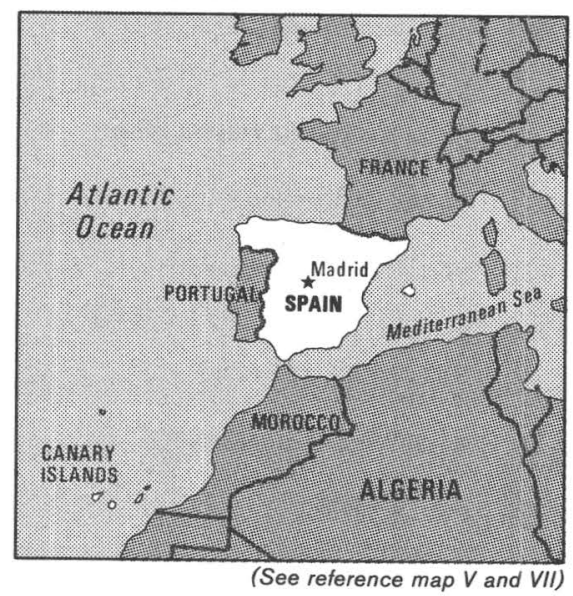

G

Servicio de Geología

Ministerio de Obras Públicas y Urbanismo

Avenida de Portugal, 81

Madrid 11

Director: Clemente Sáenz Ridruejo

G

Empresa Nacional ADARO de Investigaciones Mineras, S.A.

Serrano, 116

Director General: Santiago Navarro Bayo

G

Instituto Geológico y Minero de España

Ríos Rosas 23

Madrid 3

Director: José Enrique Azcárate Martín

G

Ministerio de Industria y Energía
G

G

G

Instituto de Geología

José Gutiérrez Abascal, 2

Madrid 6

Director: Vicente Araña Saavedra

Instituto de Investigaciones Geológicas "Jaime Almera"

Alcarria, s/n

Barcelona 28

Director: Prof. Angel López Soler

Instituto de Geología Economica

Facultad de Ciencias Geológicas

Universidad Complutense

Madrid 3

Director: José Ramón Peláez Pruneda

G

Departamento de Investigaciones Geológicas

Universidad de Granada

Facultad de Ciencias

Granada

Director:. Prof. Juan Antonio Vera Torres

G

G

H

H

C

C

Departamento de Investigaciones Geológicas

Universidad de Oviedo

Laboratorio de Geología

Escuela Superior de Minas

Independencia 13

Oviedo

Director: José Antonio Martínez Alvarez

Departamento de Investigationes Geológicas

Universidad de Salamanca

Facultad de Ciencias

Salamanca

Director: Prof. Luis Carlos García de Figuerola

Coordinacion de Hidrológica

Comisaría Central de Aguas

Nuevos Ministerios

Madrid 3

Chief:. Ignacio de Jove Ramírez

Instituto de Hidrología

Paseo Bajo Virgen del Puerto, 3

Madrid 5

President: Rafhael Heras Rodríguez

Dirección General del Instituto Gẹográfico Nacional

General Ibáñez Ibero, 3

Madrid 3

Director General: Julio Morencos Tevar

Real Sociedad Geográfica

Paseo de la Castellana, 160

Madrid 16

Valverde 22

Madrid 13 


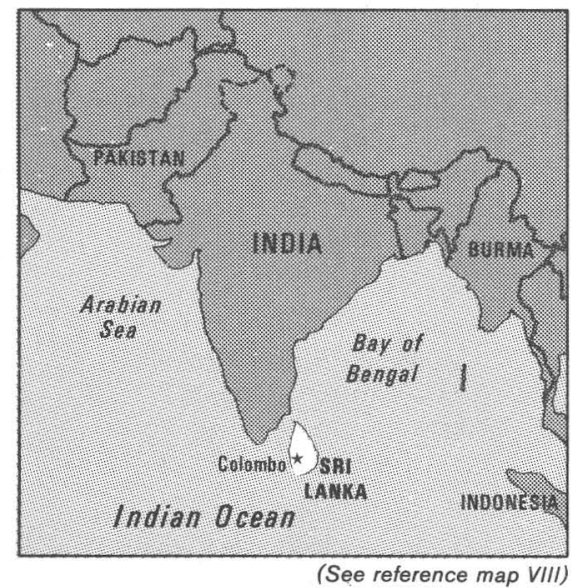

G

$\mathbf{R}$

$\mathrm{H}$

$\mathrm{H}$

C

$\mathbf{R}$
Geological Survey Department

48 Sri Jinaratna Road

Colombo 2

Director: D. J. A. C. Hapuarachchi

State Mining and Mineral Development

Corporation

63 Elvitagala Mawatha

Colombo 8

Chairman: H. Dissanayake

Hydrology Section

Irrigation Department

P.O. Box 1138

Colombo 7

Chief Engineer: N. M. G. Navaratne

Water Resources Board

2A Gregory's Avenue

Bagatelle Road

Colombo 7

Chairman: Gamini Kannangara

Surveyor-General's Office

Kirula Road

Colombo 5

Surveyor-General: S. D. F. C. Nanayakkara

Ceylon Mineral Sands Corporation

167 Sri Vipulasena Mawatha

Colombo 10

Chairman: R. S. Sirisena

Ceylon Petroleum Corporation

113 Galle Road

Colombo 3

Chairman: Daham Wimalasena

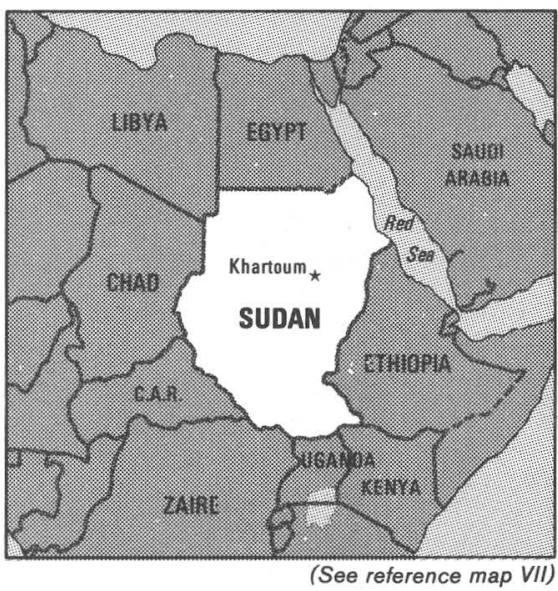

G,R

Geological and Mineral Resources

Ministry of Energy and Mining

Geological Survey Department

P.O. Box 410

Khartoum

Director: Abdalla Hassan Ishag

H

Hydrology Division

Ministry of Agriculture and Irrigation

Niles Waters Department

P.O. Box 878

Khartoum

Director: Isam Mustafa

Rural Water Administration Ministry of Energy and Mining P.O. Box 2087

Khartoum

Director General: Khairalla Mahgoub

C

Ministry of Internal Affairs

Survey Department

P.O. Box 306

Khartoum

General Director: Dafalla Salih 


\section{SURINAME}

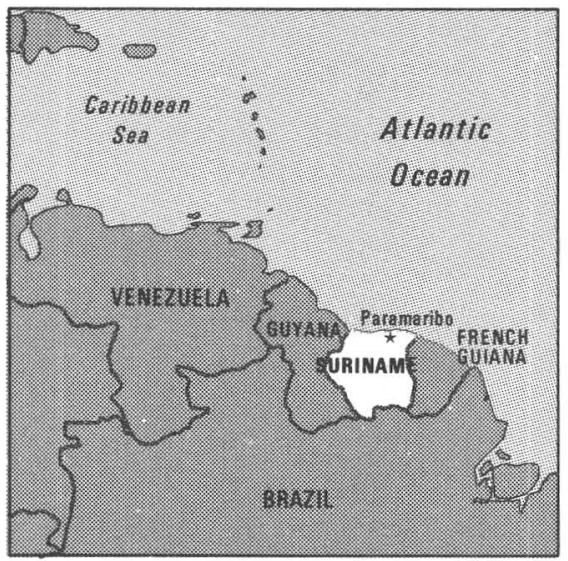

(See reference map IV)

G

Geologisch Mijnbouwkundige Dienst

Kleine Waterstraat 2-6

Paramaribo

Director: L. de Rooy

H

Water Supply Service

Ministry of Natural Resources and Energy

Kleine Combeweg 15

Paramaribo

Head: E. Tsai Meu Cheong

$\mathrm{H}$

Hydrologic Research Division

Ministry of Public Works, Telecommunication and Architecture

Verlengde Coppenamestraat

Paramaribo

Head: B. Campcens

C Centraal Bureau Luchtkaartering

P.O. Box 971

Dr. Sophie Redmondstraat 131

Paramaribo

Head: R. H. Wong Fong Sang

\section{SWAZILAND}

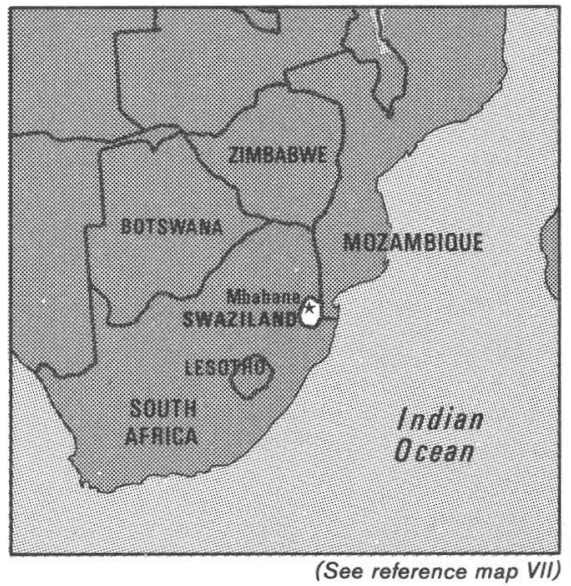

G,H,R Geological Survey and Mines Department

P.O. Box 9

Mbabane

Director: A. Dlamini

C

Department of Surveys

Ministry of Works, Power, and Communications P.O. Box 58

Mbabane

Surveyor General: G. Bishop

\section{SWEDEN}

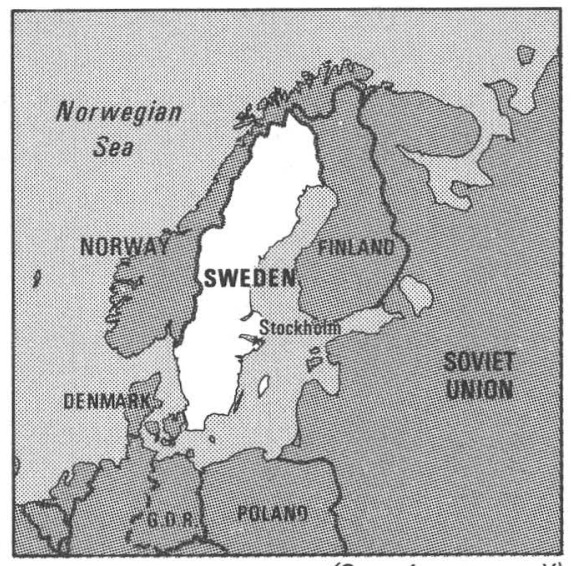

(See reference map $V$ )

G

Statens Vag-och Trafikinstitut (VTI)

Geologiska Gruppen

S-581 01 Linköping

Director General: Hans Sandebring

G

Statens Geotekniska Institut (SGI)

S-581 01 Linköping

Director: Jan Hartlén 
G,H,C,R Sveriges Geologiska Undersokning (SGU)

Box 670

[The Geological Survey of Sweden]

S-751 28 Uppsala

Director General: Arne Wesslén

C Statens Lantmäteriverk (LMV)

[The National Landsurvey of Sweden]

S-801 12 Gävle

Director General: Sten Wickbom

H

Sveriges Meteorologiska och Hydrologiska Institut (SMHI)

Box 923

S-601 19 Norrköping

Director General: Lars Ag

\section{SWITZERLAND}

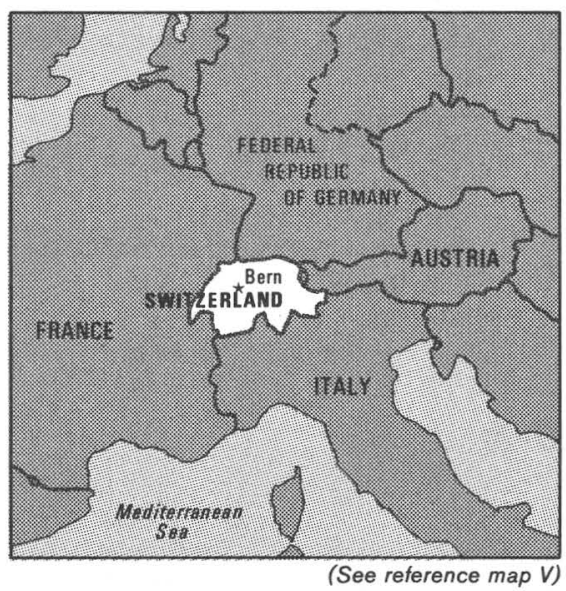

G,H Geologische Kommission der Schweizerischen Naturforschenden Gesellschaft

Birmannsgasse 9

4055 Basel

Director: H. Fischer

G

Geologisches Institut

Eidgenössische Technische Hochschule

ETH-Zentrum

8092 Zürich

Head: Prof. Dr. Rudolf Truempy

H

Eidgenössische Gewasserschutzkommission

[Federal Commission for Water Protection]

Secretariat: Bundesamt für Umweltschutz

Hallwylstrasse 4

3003 Bern

$\mathrm{H}$

Bundesamt für Wasserwirtschaft
President: Marcel Blanc

Bundesamt für Landestopographie

[Federal office for Topographical Survey]

Seftigenstrasse 264

CH-3084 Wabern

Director: Francis Jeanrichard

R

Bundesamt für Energiewirtschaft

[Federal Energy Agency]

Kapellenstrasse 14

3011 Berne

Director: E. Kiener

\section{SYRIA}

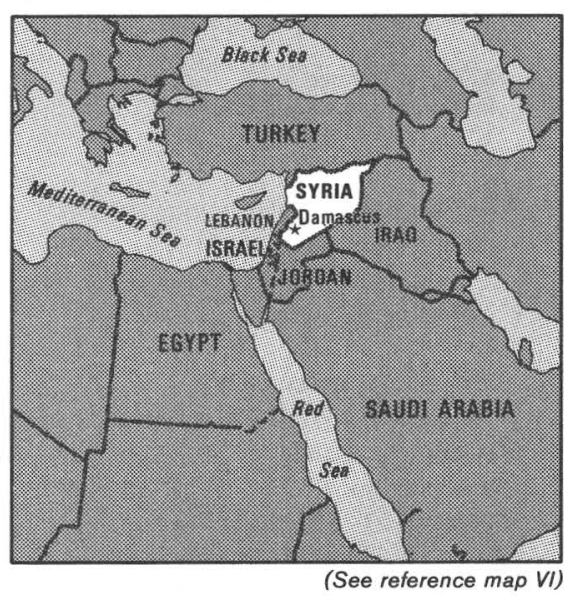

G

$\mathrm{H}$

C

C

[Federal Office for Water Economy]

Hallwylstrasse 4

3003 Bern

Director: Dr. Rodolfo Pedroli
General Establishment of Geology and Mineral Resources

Ministry of Petroleum

P. O. Box 7645

Damascus

Director General: Dr. Anwar Iman

Irrigation and Water Resources Department

Ministry of Public Works and Hydraulic Resources

Al Furat Street

Damascus

Director: Dr. Nizar Mir

Service Géographique de l'Armée

Ministry of Defense

P.O. Box 3094

Damascus

Director General: Eng. Abd Al-Mu'een Safi

National Remote Sensing Center

Ministry of Electricity

Sultan Salim St.

Minister: Dr. Eng. Ahmad Omar Yussef
Khatib Street, Adawi 
TANZANIA

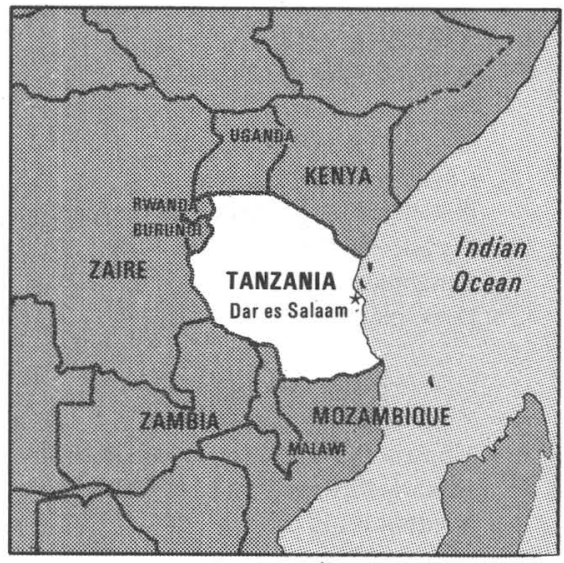

(See reference map VII)

\section{Geology}

Ministry of Minerals

P.O. Box 903

Dodoma

Commissioner for Geology:. James K. L. Saria

Survey and Mapping Division

Ministry of Lands, Housing and Urban Development

P.O. Box 9201

Dar es Salaam

Commissioner for Survey and Mapping:

$$
\text { E. N. Njau }
$$

Mapping Production

Ministry of Lands, Housing and Urban Development

P.O. Box 9301

Dar es Salaam

Senior Surveyor: O. Andrew

Hydrological Section

Ministry of Minerals

P.O. Box 903

Dodoma

Principal Mining Engineer: A. K. Muze

Principal Mineral Dressing Engineer: S. J. Mohamed

Principal Petrologist: J. N. Mushi

Principal Geologist-Mineral Exploration: W. S. Lyimo

Principal Geologist: A. M. Mwakinga
$\mathbf{R}$

Mines

Ministry of Minerals

P.O. Box 2000

Dar es Salaam

Commissioner for Mines: A. J. Zidikheri

$\mathbf{R}$

Tanzania Petroleum Development Corporation

Ministry of Water and Energy

P.O. Box 2774

Dar es Salaam

General Manager: Sylvester Barongo

\section{THAILAND}

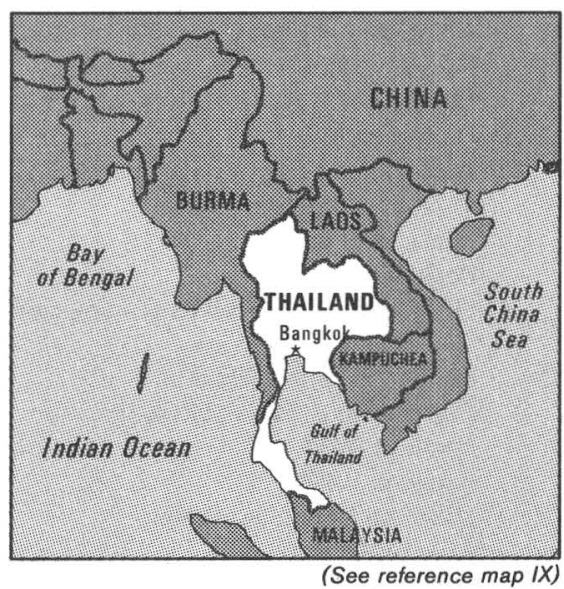

G,H Royal Forestry Department

Ministry of Agriculture and Cooperatives

Paholyothin Road

Bangkok 10900

Director-General: Pong Sono

G,H Watershed Management Division

Royal Forest Department

Ministry of Agriculture and Cooperatives

Paholyothin Road

Bangkok 10900

Director: Udhai Chanphaka

G,H Meteorological Department

Ministry of Communications

612 Sukhumvit Road

Bangkok 10110

Director-General: Dr. Boonsorn Boonsukha

G

Agrometeorology Division

Meteorological Department

Ministry of Communications

612 Sukhumvit Road

Bangkok 10110

Director: Sr. Lt. Manas Thummanond, Rtn 
Hydrometeorology Division

Meteorological Department

Ministry of Communications

612 Sukhumvit Road

Bangkok 10110

Director: Sr. Lt. Jate Chailapo, Rtn

G

Climatology Division

Meteorological Department

Ministry of Communications

612 Sukhumvit Road

Bangkok 10110

Director: Sr. Lt. Sukit Yensuang

G

Studies and Research Division

Meteorological Department

Ministry of Communications

612 Sukhumvit Road

Bangkok 10110

Director: Lt. Cdr. Sumeth Hinsheranan, Rtn.

R,G,H,C Department of Mineral Resources

Ministry of Industry

Rama VI Road

Bangkok 10600

Director-General: Sivavong Changkasiri

G,C Geological Survey Division

Department of Mineral Resources

Rama VI Road

Bangkok 10600

Director: Thawat Japakasetr

R,G Mineral Fuels Division

Department of Mineral Resources

Rama VI Road

Bangkok 10600

Director: Prakong Polhan

$\mathbf{R}$

Economic Geology Division

Development of Mineral Resources

Rama VI Road

Bangkok 10600

Director: Thawisak Danusawad

H Ground Water Division

Department of Mineral Resources

Rama VI Road

Bangkok 10600

Director: Suwit Watanachan

G,H,C Royal Irrigation Department

Ministry of Agriculture and Cooperatives

811 Samsen Road

Bangkok 10300

Director-General: Sunthorn Ruanglek

G,H Hydrology Division

Royal Irrigation Department

Ministry of Agriculture and Cooperatives

811 Samsen Road

Bangkok 10300

Director: Suthep Tingsabhat
Topographical Survey Division

Royal Irrigation Department

Ministry of Agriculture and Cooperatives

Tivanondh Road, Amphoe Pakkred

Nonthaburi 11120

Director: Phyool Chantasiro

Soil and Geology Division

Royal Irrigation Department

Ministry of Agriculture and Cooperatives

811 Samsen Road

Bangkok 10300

Director: Prasarn Leelasorn

G

Research and Laboratory Division

Royal Irrigation Department

Ministry of Agriculture and Cooperatives

Tivanondh Road, Amphoe Pakkred

Nonthaburi 11120

Director: Jumsak Tejasen

G,H Department of Public Works

Ministry of Interior

Pan Fah Bridge, Larnluang Road

Bangkok 10100

Director-General: Tongchul Singhakul

G

$\mathrm{H}$

C

C

Research and Survey Section

Construction Division

Department of Public Works

Ministry of Interior

Pan Fah Bridge, Larnluang Road

Bangkok 10100

Chief: Prasop Krasaesindhu

Provincial Water Works Division

Department of Public Works

Ministry of Interior

Pan Fah Bridge, Larnluang Road

Bangkok 10100

Director: Wiyom Niyamanusorn

Royal Thai Survey Department

Supreme Command Headquarters

Ministry of Defense

Rachinee Road

Bangkok 10200

Director: Lt. Gen. Swasdi Pachimkul

National Research Council of Thailand

Ministry of Science, Technology and Energy

196 Paholyothin Road

Bangkok 10900

Secretary-General: Dr. Choompol Swasdiyakorn

C Remote Sensing Division

National Research Council of Thailand

Ministry of Science, Technology and Energy

196 Paholyothin Road

Bangkok 10900

Director: Suvit Vibulsreth 
Defense Energy Department

Office of the Permanent Secretary of State

Ministry of Defense

496 Phetburi Road

Bangkok 10400

Director-General: Lt. Gen. Jirayute Kraisoraphong

$\mathbf{R}$

Raw Material Exploration and Production Division

Defense Energy Department

Office of the Permanent Secretary of State

Ministry of Defense

496 Phetburi Road

Bangkok 10400

Chief:. Col. Piroj Chuenpratum

$\mathrm{H} \quad$ Mekong Committee

c/o ESCAP, United Nations Building

Rajdamnoen Avenue

Bangkok 10200

C,H,G National Energy Administration

Ministry of Science, Technology and Energy

Kasatsuk Bridge

Bangkok 10500

Secretary-General: Prapath Premmani

$\mathrm{C}, \mathrm{H}, \mathrm{G} \quad$ Energy Investigation Division

National Energy Administration

Ministry of Science, Technology and Energy

Kasatsuk Bridge

Bangkok 10500

Director: Suwat Sanguanwongse

C

Surveying and Mapping Branch

Energy Investigation Division

National Energy Administration

Ministry of Science, Technology and Energy

Kasatsuk Bridge

Bangkok 10500

Chief: Ararm Supakarn

H

Hydrology Branch

Energy Investigation Division

National Energy Administration

Ministry of Science, Technology and Energy

Kasatsuk Bridge

Bangkok 10500

Chief: Borom Somsub

G

Engineering Geology Branch

Energy Investigation Division

National Energy Administration

Ministry of Science, Technology and Energy

Kasatsuk Bridge

Bangkok 10500

Chief: Sompongs Sriphyak

R,G Petroleum Authority of Thailand

14 Soi Yasoob, Vibhavadi Rangsit Road

Bangkok 10900

Governor: Dr. Tongchat Hongladaromp
R,G Peteolrum Exploration and Production Department

Petroleum Authority of Thailand

14 Soi Yasoob, Vibhavadi Rangsit Road

Bangkok 10900

Director: Preecha Supalak

$\mathbf{R}$

Drilling and Production Division

Petroleum Exploration and Production Department

Petroleum Authority of Thailand

14 Soi Yasoob, Vibhavadi Rangsit Road

Bangkok 10900

Chief: Maroot Mrigadat

R Petroleum Geology Division

Petroleum Exploration and Production Department

Petroleum Authority of Thailand

14 Soi Yasoob, Vibhavadi Rangsit Road

Bangkok 10900

Chief: Prisdapunt Pojanapreecha

G Geophysics Division

Petroleum Exploration and Production Department

Petroleum Authority of Thailand

14 Soi Yasoob, Vibhavadi Rangsit Road

Bangkok 10900

Chief: Chalee Siriratanamongkol

G

Land Development Department

Ministry of Agriculture and Copoeratives

Paholyothin Road

Bangkok 10900

Director General: Anunt Komes

G

Soil Survey Division

Land Development Department

Ministry of Agriculture and Cooperatives

Paholyothin Road

Bangkok 10900

Director: Samarn Panichapong 


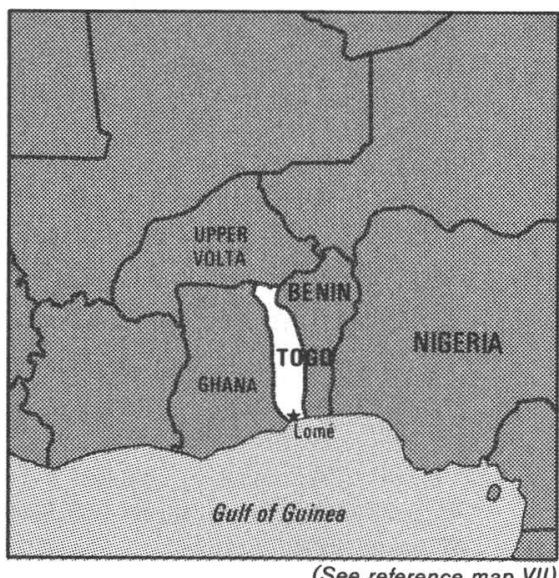

(See reference map VII)

G,H Direction Générale des Mines, de la Géologie et du Bureau National de Recherches Minières

B.P. 356

Lomé

Directeur: N'zoulou Péré

G,H Département Hydro-géologie

Ministère des Mines de l'Energie, des

Ressources Hydrauliques et des Travaux

Publics

B.P. 356

Lomé

Chief:. Comlanvi N. D'Almeida

G Direction de la Pédologie

Ministère de l'Aménagement Rural

B.P. 1026

Lomé

Directeur: Koffi Lomko Allaglo

G,H Office de la Recherche Scientifique et Technique Outre-Mer Centre (ORSTOM), Office de Lomé ${ }^{10}$

B.P. 375

Lomé

Directeur: Bernard Huguenin

C Service Topographique

Ministère des Mines, de l'Energie, des Ressources Hydrauliques et des Travaux Publics

B.P. 500

Lomé

Directeur: Issa-Gnon Gbarré

\footnotetext{
${ }^{10}$ French research organization; no governmental affiliation.
}

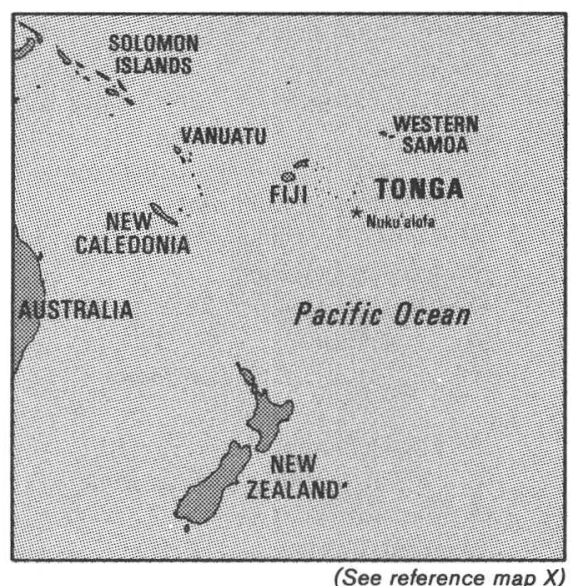

G,C,H, R Ministry of Lands, Survey and Natural Resources P.O. Box 5

Nuku'alofa

Superintendent: S. L. Tongilava

\section{TRINIDAD AND TOBAGO}

(No recent information)

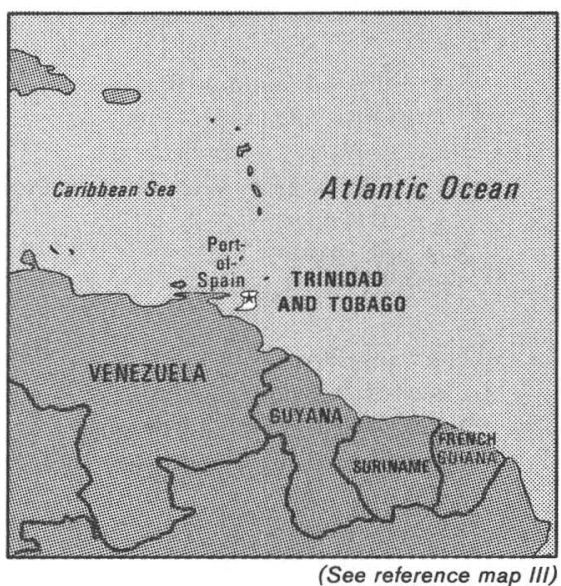

$\mathrm{G}, \mathrm{R}$

Ministry of Energy and Energy-Based Industries P.O. Box 96

Port-of-Spain, Trainidad

Permanent Secretary: G. H. Legall

Senior Geologist: John P. Scott

Water Resources Agency

P.O. Box 145

Port-of-Spain, Trinidad

Director: Elton Wyke 
Port-of-Spain, Trinidad

Director of Surveys: Peter Samuel

\section{TUNISIA}

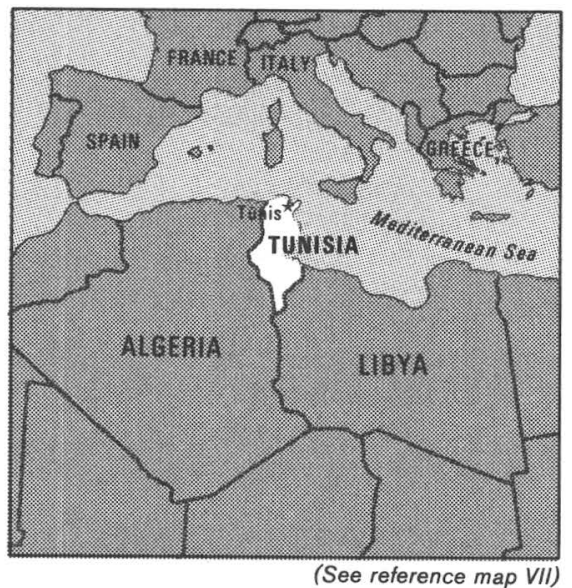

G

$\mathrm{H}$

Ministère de l'Agriculture

Division des Ressources en Eau

30 Rue Alain Savary

Tunis

Director: Ahmed Khouaja

Ministère de l'Agriculture

Direction des Études et Grands Travaux

Hydrauliques

30 Rue Alain Savary

Tunis

Director: Ameur Horchani

Office de la Topographie et de la Cartographie Cité Olympique

Tunis

President Director General: Habib Amri

Entreprise Tunisienne des Activités Petroliéres (ETAP)

11 Avenue Khereddine Pacha

Tunis

President Director General: Habib Lazreg

Office National des Mines
26 Rue d'Angleterre

Tunis

President Director General: Ali Attya
$\mathbf{R}$

R,G Ministere de l'Économie Nationale

Directon des Mines et de la Géologie

7 Rue du Royaume d'Arabie Seoudite

Tunis

Director of Mines and Geology: Mohsen Zerelli

\section{TURKEY}

$\mathrm{G}, \mathrm{C}, \mathrm{H}$

$\mathrm{H}$

C

Ministère de L'Économic Nationale

195 Rue de la Kasbah

Tunis

Director of Energy: Mohamed Sahbi M'rabet

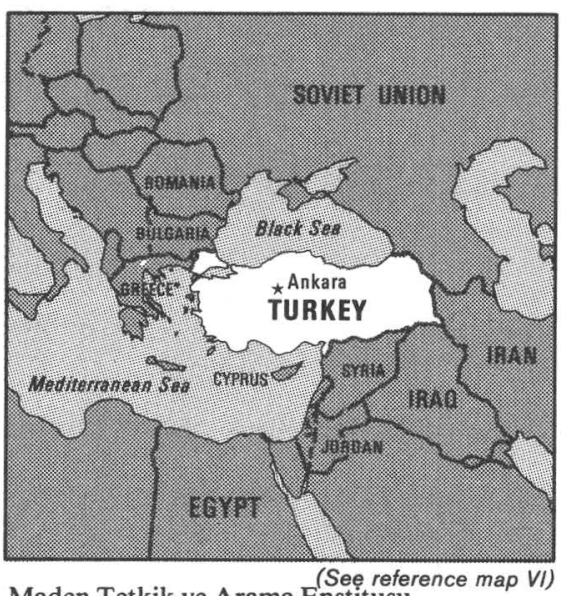

Maden Tetkik ve Arama Enstitusu

[Mineral Research and Exploration Institute]

Eskisehir Yolu Ustu-Ankara

Director General: M. Sitki Sancar

Devlet Suisleri Genel Mudurlugu

[State Hystaulid Works (DSI)]

Eskisehir Yolu-Yucetepe-Ankara

Director General: Sabahattin Sayin

T.C., M.S.B. Harita Genel Mudurlugu

[General Directorate of Turkish Mapping Service]

Ministry of Defense

Cebecci-Ankara

Director General: General Sabri Delic

$\mathrm{R}, \mathrm{G}$

Petrol Ofisi Genel Mudurlugu

[Petroleum Administration]

Bestekar Sokak No. 18, Ankara

Yevishehir, Ankara

President: Ruhi Orbay

$\mathbf{R}$

Enerji ve Tabii Kaynaklar Bakanligi

[Ministry of Energy and Natural Resources]

Bakanliklar-Ankara

Minister: Fahir Ilkel

Imar ve Iskan Bakanligi

[Ministry of Reconstruction and Settlement] Earthquake Research Institute

Deprem Arastirma Dairesi

Eskisehir Yolu-Ankara

Director: Oktay Ergundy 


\section{UGANDA}

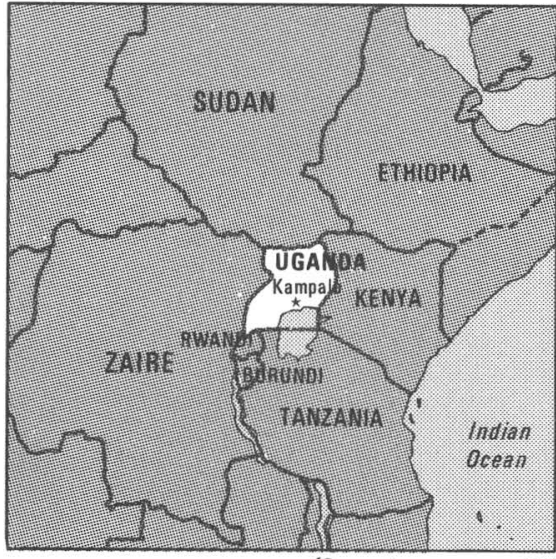

(See reference map VII)

G

C

$\mathrm{H}$

Geological Survey and Mines Department P.O. Box 9

Entebbe

Acting Commissioner: Saul Mboijana

Department of Lands and Surveys

P.O. Box 7061

Kampala

Commissioner: Paul Bakashabaruhanga

Ministry of Minerals and Water Resources

Water Development Department

P. O. Box 19

Entebbe

Acting Commissioner:. E. A. Oryang

\section{UNITED KINGDOM}

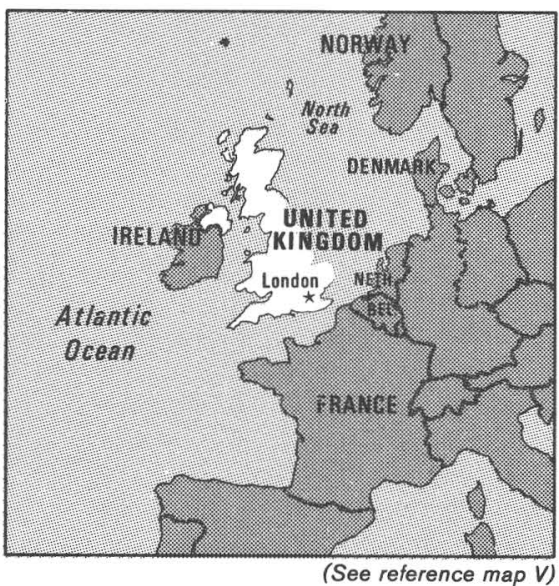

G

Institute of Oceanographic Sciences

Wormley, Godalming

Surrey GU8 5UB

Director: A. S. Laughton
British Geological Survey

Exhibition Road

London, SW7 2DE

Deputy Director: P. A. Sabine

Overseas Division

Nieker Hill

Keyworth

Nottingham NG 12 5GG

Director: G. M. Brown

G,C Directorate of Overseas Surveys

Foreign and Commonwealth Office

Kingston Road

Tolworth, Surbiton

Surrey KT5 9NS

Director:. B. S. Surmston

The British Antarctic Survey

Madingley Road

Cambridge CB3 OET

Director: R. M. Laws

Water Data Unit

Department of the Environment

Reading Bridge House

Reading RG1 8PS

Director: D. H. Newsome

C

Ordnance Survey

Ramsey Road

Maybush

Southampton SO9 4DH

Director General: Walter P. Smith

C

Directorate of Military Survey

Ministry of Defence

Elmwood Avenue

Feltham, Middlesex TW13 7AE

Director: Major-General E. W. Barton, MBE

$\mathrm{H}$

Institute of Hydrology

Natural Environment Research Council

Maclean Building, Crowmarsh Gifford

Wallingford

Oxfordshire OX10 8BB

Director: J. S. G. McCulloch 
UNITED STATES OF AMERICA

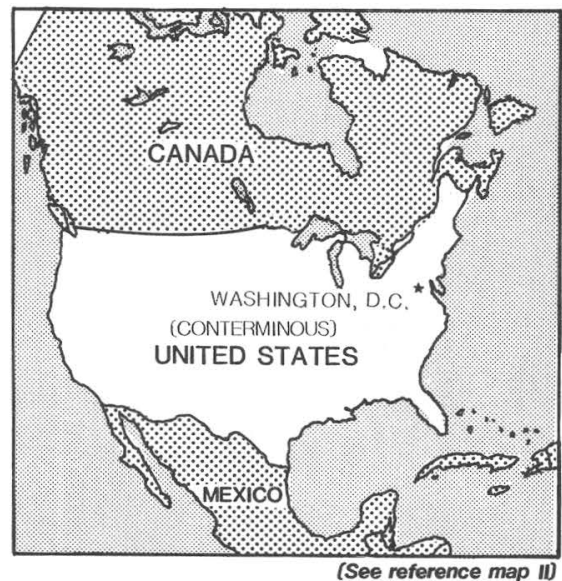

G

U.S. Bureau of Mines (USBM)

2401 E. Street, NW

Washington, D.C. 20241

Director: Robert C. Horton

G,C,H,R U.S. Geological Survey (USGS)

National Center

12201 Sunrise Valley Drive

Reston, Virginia 22092

Director:. Dallas L. Peck

C

Defense Mapping Agency (DMA)

Building 56, Naval Observatory

Washington, D.C. 20305

Director: E. A. Wilkinson, Jr.

C

\section{UPPER VOLTA}

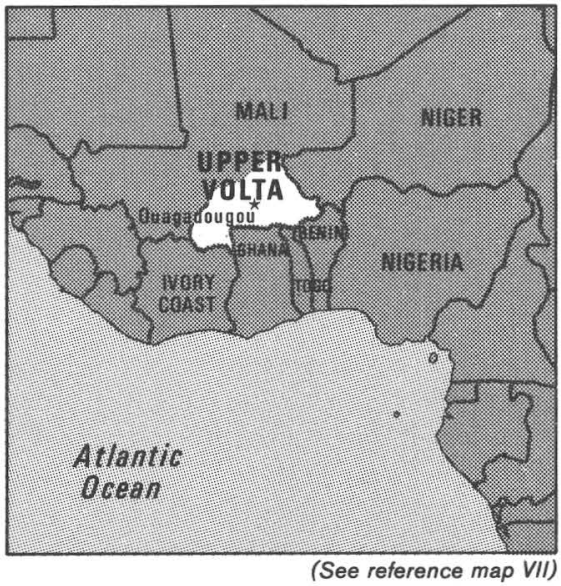

G

Bureau Voltaique de la Géologie et des Mines B.P. 601

Ouagadougou

Director: Emile Gansonre

H

Direction de l'Hydraulique et de l'Equipement Rural

B.P. 330

Ouagadougu

Director: Victor Ouedraogo

$\mathrm{H}$

Office de la Recherche Scientifique et Technique Outre-Mer (ORSTOM) ${ }^{11}$, Centre Ouagadougou

B.P. 182

Ouagadougou

Director: Jacques Claude

National Oceanic and Atmospheric Administration (NOAA)

H

Department of Commerce

Rockville, Maryland 20852

Administrator: John V. Byrne
Comité Inter-Africain d'Etudes Hydrauliques (CIEH)

B.P. 369

Ouagadougou

Secretary-General: Mr. Hassan

C Direction du Cadastre et Topographie Service Topographique

Ministère des Finances et du Commerce

B.P. 7054

Ouagadougou

Director: Omar Traore

\footnotetext{
${ }^{11}$ French research organization; no governmental affiliation.
} 


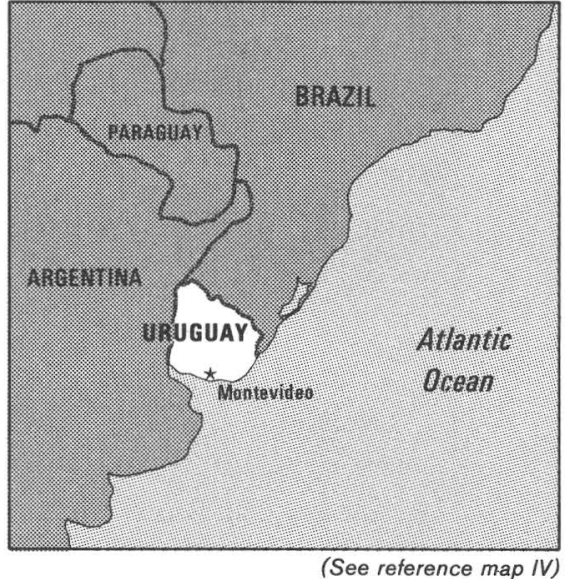

G

G

$\mathrm{H}$

C

C

C
Instituto Geológico del Uruguay

Hervidero 2853

Montevideo

Director: Coronel Mario E. Latorre

Instituto Nacional de Pesca (INAPE)

Constituyente 1497

Montevideo

Director: Ulises W. Perez

Direccion de Hidrografía

Ministerio de Transporte and Obras Publicas

Ciudadela 1414

Montevideo

Director: Capitan de Navio Tabare Estevez

Servicio Geográfico Militar

Avenida 8 de Octubre 3255

Montevideo

Director: Coronel Ivho R. Acuna

Direccion de Topografía

Ministerio de Transporte and Obras Públicas

Rincón 561 2d Piso

Montevideo

Director: Coronel Alberto Ortiz

Servicio de Hidrografía de la Marina

Capurpo 980

Montevideo

Director: Capitán de Navio James Coates

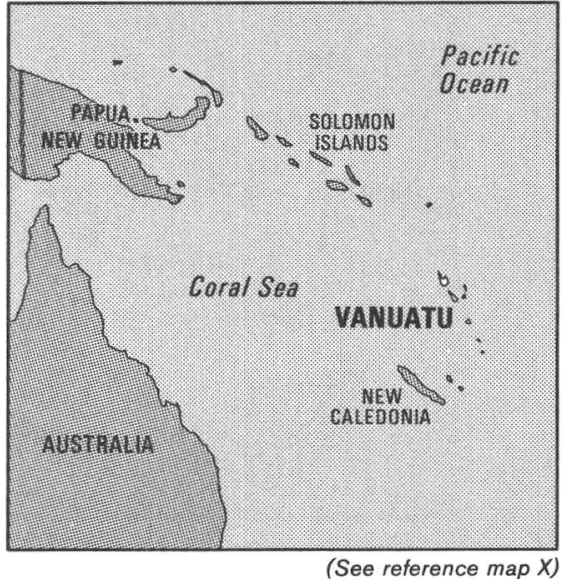

G

Geological Survey

British Residency

Port-Vila

Director: A. MacFarlane

G

Mineral Resources Department

B.P. 637

Port-Vila

Director: J. L. Saos

\section{VENEZUELA}

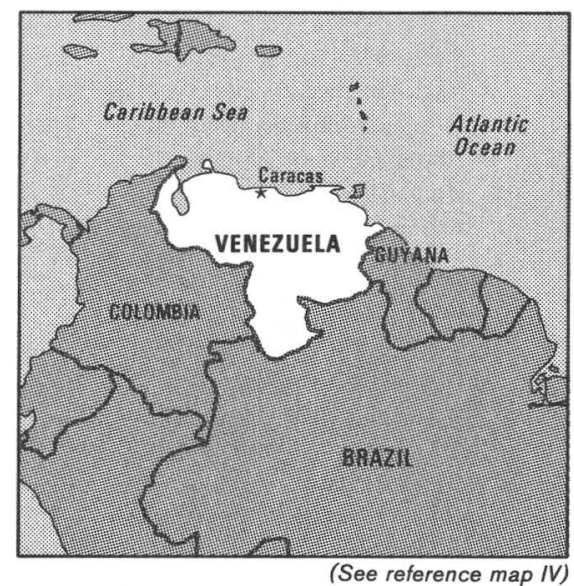

Dirección de Investigáciones Geoanalísticas y Tecnológicas

Edificio Guayana, Piso 1

Calle 10 - La Urbina

Caracas

Director: Dr. Ángel Fonseca A. 
Fundación Venezolana de Investigáciones

Seismológicas

Avenida Wáshington

Quinta Francinetti

Urb. San Bernardino

Caracas

Presidente: Dr. Alirio Bellizia

G,R

Dirección General Sectorial de Minas y Geología

Ministerio de Energía y Minas

Torre Oeste, Piso 4

Parque Central

Caracas

Director General: Dr. Marcial Merino Valery

G,R Dirección de Minas

Dirección General Sectorial de Minas y Geologia

Ministerio de Enérgía y Minas

Torre Oeste, Piso 6

Parque Central

Caracas

Director: Dr. Rafael Borges

G,R Dirección de Geología

Dirección General Sectorial de Minas y Geología

Ministerio de Energía y Minas

Torre Oeste, Piso 4

Parque Central

Caracas

Director: Dr. F. Rául García Jarpa

G,R Consejo Consultivo de Directores de Servicios Geológicos de Latinoamérica

Torre Oeste, Piso 5

Parque Central

Caracas

C

Dirección de Cartografía Nacional

Edificio Camejo, Piso 2, Ofic. 230

Centro Simón Bolívar

Caracas

Director: Dr. José Clemente Pérez Oráa

R

Dirección General Sectorial de Hidrocarburos

\section{VIETNAM}

(No recent information)

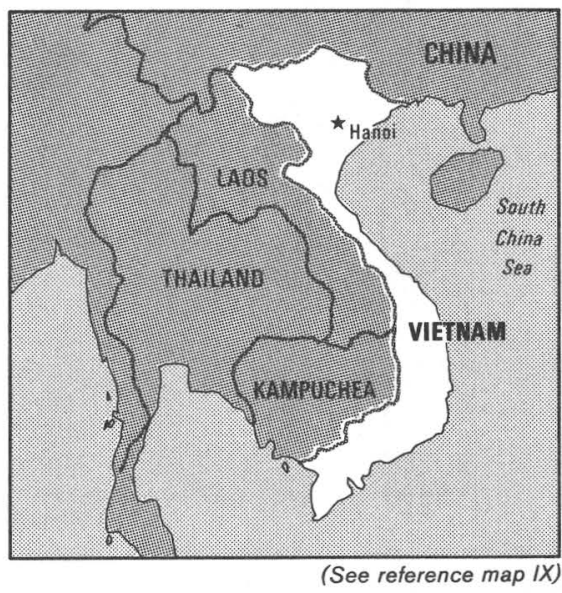

G

Geologic Section

State Committee of Sciences

Hanoi

G State Committee of Sciences

Station of Oceanography

Hanoi

H Service Hydrometeorologique

4 rue Dang Thai Than

Hanoi

Directeur General: Tran Van An

C

Service Geographique Nationale

Ministere de la Defense Nationale

Dalat

Director: Nguyen Van Khai

Ministerio de Energía y Minas

Torre Oeste, Piso 12

Parque Central

Caracas

Director General: Dr. Jesús García Coronado 


\section{WESTERN SAMOA}

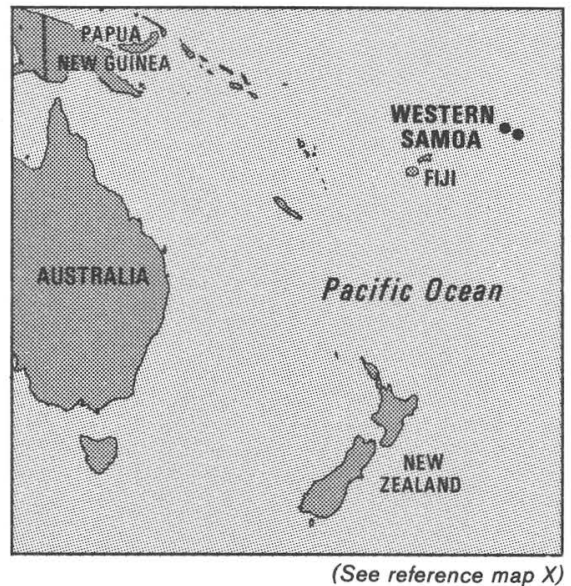

$\mathrm{C}$

Lands and Survey Department

Main Beach Road

P.O. Box 63

Apia

Director of Lands: Arthur P. Hunter

H

Apia Observatory

P.O. Box 52

Apia

Superintendent: L. Ionae

\section{YEMEN, ARAB REPUBLIC}

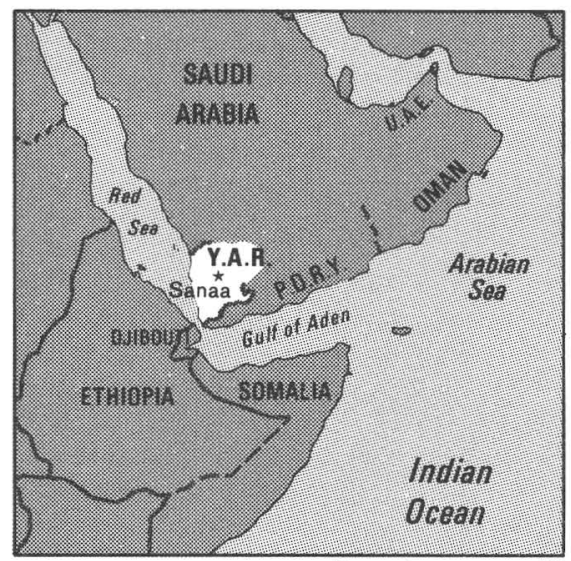

(See reference map VI)

G

Central Planning Organization

P.O. Box 175

Sanaa

G, R Geological Authority

Yemen Oil and Mineral Resources Corporation (YOMINCO)

P.O. Box 81

Sanaa

Director-General: Ali Jaber Alawi
Department of Hydrology (YOMINCO)

Sanaa

Director-General: Ali Jaber Alawi

\section{YEMEN, PEOPLE'S DEMOCRATIC REPUBLIC OF}

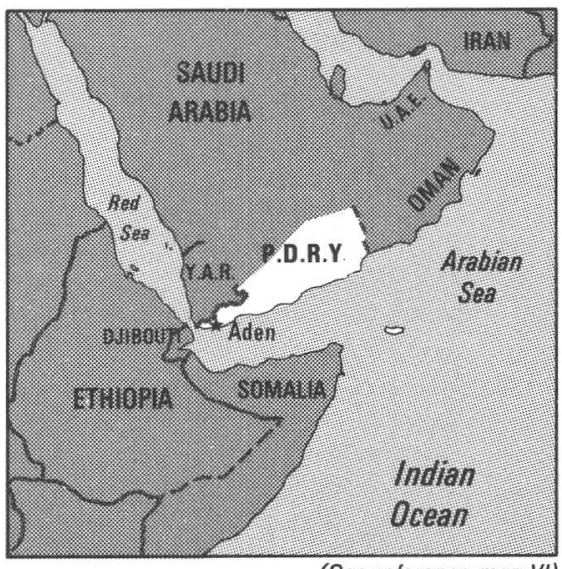

G

Overseas Geological Surveys of London, England c/o Aden Public Works Department Aden

G,H,C Ministry of Public Works Khormaksar, Aden

\section{YUGOSLAVIA}

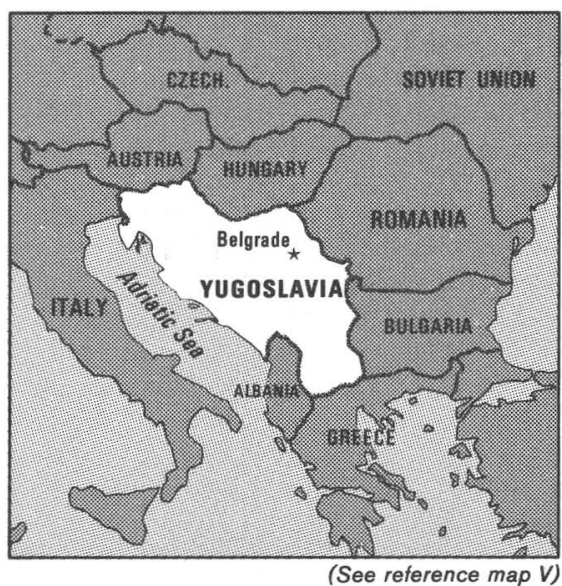

G

Zavod za geoloska i geofizicka istrazivanja (GEOZA VOD) [Institute for Geological and Geophysical Research]

48, Karadjordjeva

11000 Belgrade

Director General: Petar Vojnovic 
G,H Institut za hidrogeologiju i hidrotehniku(GEOINZINJERING) [Institute for Hydrogeology and Hydrotechnology]

3, Ustanicka

71000 Sarajevo

Director General: Stanko Celikovic

H

$\mathrm{H}$

C

G, R Institute za zemljotresno inzenjerstvo i inzenjersku seizmologiju (IZIIS) [Institute for Earthquake Engineering and Engineering Seismology]

P.O. Box 101

91000 Skopje

Director General: Jakim Petrovski

G,H,C, R Savezni zavod za medjunarodnu naucnu, prosvetnu, kulturnu i tehnicku saradnju (YUZAMS) [Federal Administration for International Scientific, Educational, Cultural and Technical Cooperation- FAISECTC]

29, Kosancicev venac

11000 Belgrade

Director General: Miljenko Zrelec

\section{ZAIRE}

(No recent information)

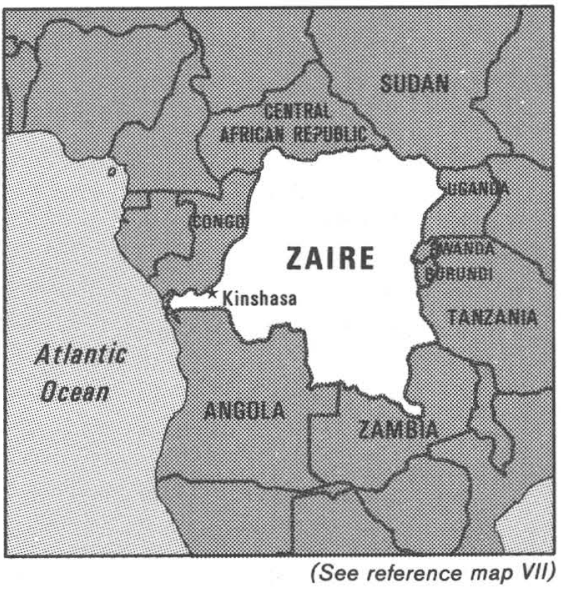

G

Service Géologique du Zaire

Ministry of Mines

B.P. 898

44 Avenue des Huileries

Kinshasa

Director: Mubiayi

$\mathrm{H}$

Regie des Voies Navigables

B.P. 3144

Kinshasa

Directeur: M. Ndombasi

C

Institut Géographique du Zaire

106 Blvd du 30 Juin

B.P. 3086

Kinshasa

Director: Lubiku Lusiense Belani

\section{ZAMBIA}

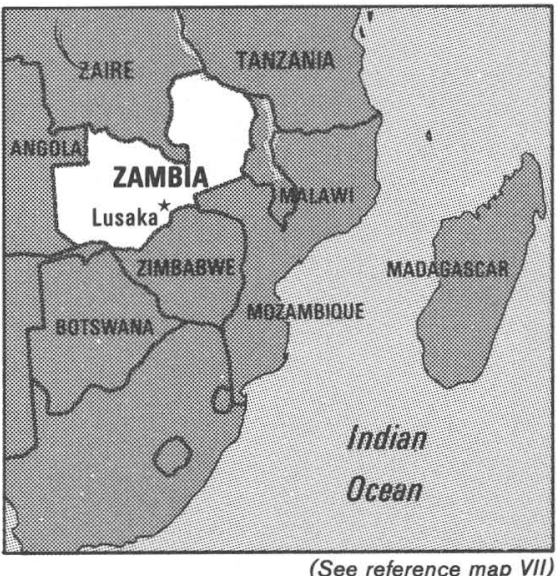

Geological Survey Departmient Ministry of Mines P.O. Box 50135 Lusaka Acting Director: D. Green 
G,R

Zambia Industrial and Mining Corporation, Limited (ZIMCO)

Zimco House, Cairo Road

P.O. Box 30090

Lusaka

Director General: James Mapoma

G Mining Industry and Technical Services

Zambia Consolidated Copper Mines Limited (ZCCM)

P.O. Box 10

Kalulushi

Manager: Dr. Silane Mwenechanya

C Survey Department

Ministry of Lands and Natural Resources

Mulungushi House

P.O. Box 50397

Lusaka

Surveyor General: Andrew J. Kownacki

H Water Affairs Department

Ministry of Agriculture and Water Development

Mulungushi House

P.O. Box 50288

Lusaka

Director: C. R. W. Kayombo

\section{ZIMBABWE}

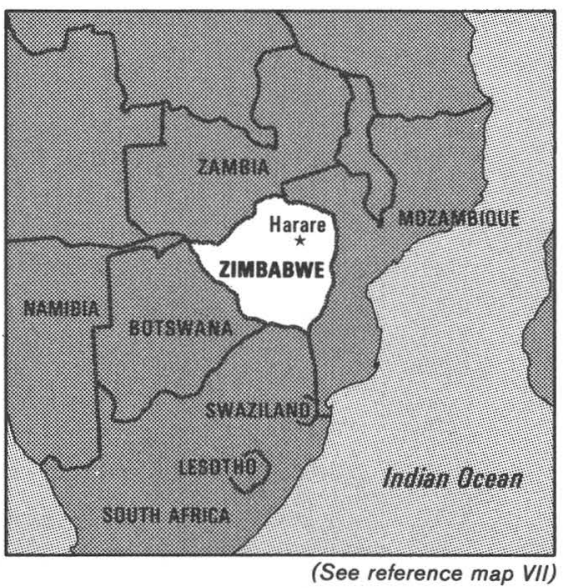

G

Department of Geological Survey

Ministry of Mines

P.O. Box 8039, Causeway

Harare

Director: E. R. Morrison

H

Chief Hydrological Engineer
C

Department of the Surveyor General

Ministry of Lands, Resettlement, and Rural Development

P.O. Box 8099, Causeway

Harare

Surveyor General: R. S. Cole

\section{TAIWAN}

(No recent information)

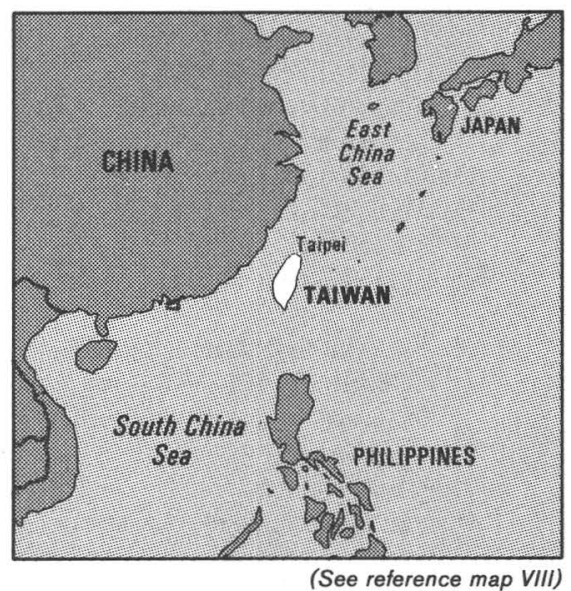

G

Chinese Petroleum Corporation

83 Chunghwa Road

Taipei

Chairman: Jerome Hu

G

G Institute of Geology

National Taiwan University

1 Roosevelt Road, Section 4

Taipei

Director: Yuan Wang

G Institute of Geophysics

National Taiwan University

Chung Li, Taoyuan County

Chairman: Chieh-hou Yang

G Institute of Earth Sciences

Academia Sinica

P.O. Box 23-59

Taipei

Director: Yi-ben Tsai

Ministry of Water Resources and Development

P.O. Box 8132, Causeway

Harare

Chief: J. C. Johnston
Central Geological Survey

P.O. Box 968

Taipei

Director: Tieh-liang Hsu 
Agriculture and Forstry Aerial Survey Team

Taiwan Provincial Forestry Bureau

101-1, Ho Ping West Road, Section 2

Taipei

Team Leader: Tse-lin Huang

C

Combined Service Forces

Ministry of National Defense

Chieh Shou Hall, Chungking South Rd.

Taipei

Commander-in-Chief: To-nien Wang

H Taiwan Provincial Water Conservancy Bureau

Nanking Road

Taichung

Director: Wen-hsiang Chen
H

Taiwan Power Company

39 Ho Ping East Road, Section 1

Taipei

Chairman: Lan-Kao Chen

H

Water Resources Planning Commission

Ministry of Economic Affairs

2, Ning Po East Street

Taipei

Chairman: Hong-hsi Hsu

R,G Mining Research and Service Organization Industrial Technology Research Institute

1 Tun Hua South Road

Taipei

Director: Tah-tsung Feng 


\section{MAJOR INTERNATIONAL ORGANIZATIONS}

American Association for the Advancement of Science (AAAS)

Section 5

1776 Massachusetts Avenue, NW

Washington, D.C. 20036 U.S.A.

Executive Secretary: William P. Carey

American Association of Petroleum Geologists (AAPG)

P.O. Box 979

Tulsa, Oklahoma 74101 U.S.A.

Executive Director: Fred A. Dix, Jr.

American Geological Institute (AGI)

4220 King Street

Alexandria, Virginia 22302 U.S.A.

Executive Director: A. G. Unklesbay

American Geophysical Union (AGU)

2000 Florida Avenue, NW

Washington, D.C. 20009 U.S.A.

Executive Director: A. F. Spilhaus, Jr.

Association of Geoscientists for International Development (AGID)

c/o Asian Institute of Technology

G.P.O. Box 2754

Bangkok 10501

Thailand

Secretary General: Tan Bock Kang

G,H,C Arctic Institute of North America (AINA)

University of Calgary

University Library Tower

2920 24th Avenue, NW

Calgary, Alberta

Canada T2N 1N4

Executive Director: John S. Tener

Centre Sismologique Europeo-Mediterraneen (EMSC)

5 , rue René Descartes

F 67084 Strasbourg Cedex

France

Director: E. Peter Schmitt

Committee for Co-ordination of Joint Prospecting for Mineral Resources in Asian Offshore Areas, East Asia (CCOP,EA)

c/o ESCAP

United Nations Building

Bangkok 2, Thailand

Manager/Chief Technical Advisor: S. K. Chung

Committee for Co-ordination of Joint Prospecting for Mineral Resources in Asian Offshore Areas, South Pacific (CCOP/SOPAC)

c/o Mineral Resources Division

Private Mail Bag

Suva, Fiji

Project Manager: Cruz A. Matos
G

G

G,C

G

R

$\mathrm{H}$

G

G, H

G 
Instituto Panamericano de Geografia e Historia (IPGH)

Comision de Geofisica del IPGH

Apartado 11363

Lima 14, Peru

Presidente: Alberto A. Giesecke M.

G,H,C International Association for Advancement of Earth and Environmental Sciences

Department of Earth Sciences

Northeastern Illinois State University

Bryn Mawr at St. Louis Avenue

Chicago, Illinois 60625 U.S.A.

Contact: Musa Qutub

H International Atomic Energy Agency (IAEA)

Wagramerstrasse 5, P.O. Box 100

A-1400 Vienna, Austria

Director General: Hans Blix

International Association of Engineering Geology (IAEG)

Laboratoire Central des Ponts et Chaussees

58, Boulevard Lafebvre

75732 Paris Cedex 15, France

Secretary General: L. Primel

C

International Association of Geodesy (IAG)

39ter, rue Gay Lussac

F-75005 Paris, France

Secretary General: M. Michael Louis

H

International Association of Hydrological Sciences (IAHS)

Maclean Building

Crownmarsh Gifford

Wallingford OW 10888

England

Secretary General: J. C. Rodda

G

International Association for Mathematical Geology (IAMG)

Kansas Geological Survey

University of Kansas

Lawrence, Kansas 66045 U.S.A.

Secretary General: John C. Davis

G

International Association of Planetology

Institute of Applied Geophysics

Podbelohorska 47

11015 Praha, Czechoslovakia

Executive Officer: Nadezda Stovickova

International Association for the Physical

Sciences of the Ocean (IAPSO)

LaFond Oceanic Consultants

P.O. Box 7325

San Diego, California 92107 U.S.A.

President: Devendra Lal

Secretary-General: Eugene C. LaFond
International Association of Sedimentologists

Laboratoire de Paléontologie

Université de Liège

7, Place du 20 Août

4000, Liége, Belgium

General Secretary: Clv Monty

G

International Association for the Study of Clays

Place Croix de Sud 1

B-1348 Louvain-la-Neuve

Belgium

Secretary-General: A. J. Herbillon

G

International Association of Seismology and Physics of the Earth's Interior (IASPEI)

Newbury RG13 1LK

England

Secretary General: R. D. Adams

G

International Association of Volcanology and Chemistry of the Earth's Interior (IAVCEI)

Department of Geology

University of Nottingham

University Park

Nottingham NG7 2RD

Secretary General: P. E. Baker

C

G

International Cartographic Association (ICA)

Fottbrovägen 16

S-11264 Stockholm, Sweden

President: F. J. Ormeling

Secretary General: $\mathrm{O}$. Hedbom

International Council for Exploration of the Sea (ICES)

Palaegade 2-4

DK-1261 Copenhagen K, Denmark

Secretary General: Hans Tambs-Lyche

President: B. B. Parrish

International Commission on Irrigation and Drainage (ICID)

48, Nyaya Marg, Chanakyapuri

New Delhi-110 021

India

International Commission on Large Dams (ICOLD)

151, Boulevard Haussmann

75008 Paris, France

Secretary General: Joannes Cotillon

International Commission for Palynology

Esso Rep-EPR-E

B.P. 150

33321 Begles Cedex, France

Secretary-Treasurer: R. Jan du Chene

G,H,C International Council of Scientific Unions (ICSU)

51, Boulevard de Montmorency

75016 Paris

France

Executive Secretary: F. W. G. Baker 
The World Bank

1818 H Street, NW

Washington, D.C. 20433 U.S.A.

International Energy Agency (IEA)

World Coal Resources and Reserves Data Bank Service

14, Lower Grosvenor Place

London SW1W OEX, England

G

G

G

$\mathbf{G}$

C International Hydrographic Organization (IHO)

International Coordination Council of Societies of Mineral Deposits Geology (CCSMDG)

Departamento de Geologia

Universidad Autonoma

Canto Blanco

Madrid 34, Spain

Secretary Treasure: G. Monseur

International Geological Correlation Programme (IGCP)

UNESCO

7, Place de Fontenoy

75700 Paris, France

Secretary: E. von Braun

International Geological Congress, 27th, 1984 (IGC)

Institute of Lithosphere

22, Staromonetny per

109180 Moscow, U.S.S.R.

President of Organizing Committee, Y. A. Kozlovsky

Secretary General: N. A. Bogdanov

International Glaciological Society

c/o Scott Polar Research Institute

Lensfield Road

Cambridge, CB2 1ER, England

Secretary General: Hilda Richardson

G,C International Geographical Union (IGU)

Geographischr Institut

University of Freiburg

D-78 Freiburg, Federal Republic of Germany

Secretary General: Walter Manshard

International Hydrographic Bureau (IHB)

7 Avenue President Kennedy

\section{B.P. 345 Monte-Carlo}

MC 98000, Monaco

Directing Committee: F. L. Fraser, President;

$$
\text { O. A. A. Affonso; J. E. Ayres }
$$

International Hydrological Programme

UNESCO, Divison of Water Sciences

7 Place de Fontenoy

75700 Paris, France

Secretary: Sorin Dumitrescu

International Institute for Applied Systems Analysis (IIASA)

Schloss Laxenburg

2362 Laxenburg, Austria

Director: C. S. Holling

G,H,C International Institute for Aerial Survey and Earth Sciences (ITC)

144 Boulevard 1945

P.O. Box 6

Enschede, Netherlands

Secretary: A. J. van aer Weele

Internatonal Mineralogical Association (IMA)

University of Marburg, Institute of Mineralogy

Lahnberge

3550 Marburg, West Germany

Secretary: Stefan S. Hafner

Intergovernmental Oceanographic Commission (IOC)

UNESCO

7, Place de Fontenoy

75700 Paris, France

Chairman: I. A. Ronquillo

Secretary: Mario de Oliviera Ruivo

International Oceanographic Foundation (IOF), Planet Ocean

3979 Rickenbacker Causeway

Miami, Florida 33149 U.S.A.

Chief Executive Officer: F. G. Walton Smith

International Ocean Institute (IDI)

Royal University of Malta

Msida, Malta

Director: G. L. Kesteven

International Organization of Paleobotany (IOP)

NE London Polytechnic

Romford Road

London E15 4L2, England

President: $T$. Delevoryas

Secretary General: M. C. Boulter

International Organization for Standardization (ISO)

1 Rue de Varembé

1211 Geneva 20, Switzerland

Secretary General: Olle Sturen

International Paleontological Association (IPA) Geologisch-Palaontologisches

Institut und Museum der Universitat Gottingen

Goldschmidt-Strasse 3

D-3400 Gottingen, Federal Republic of Germany Secretary General: H. Walliser 
International Society for Rock Mechanics (ISRM)

Laboratorio Nacional de Engenharia Civil

101, Av. do Brasil

P-1799 Lisboa CODEX, Portugal

Secretary General: Nuno F. Grossmann

G,C,H International Society for Photogrammetry (ISP)

Institut fur Photogrammetrie

Universitat Hannover

Nienburger Strasse 1

D-3000 Hannover, West Germany

Secretary General: Gottfried Konacny

G International Union of Crystallography (IUCR)

5 Abbey Square

Chester CH1 2HU, England

President: J. Karle

Executive Secretary: J. N. King

G,C,H International Union for Conservation of Nature and Natural Resources (IUCN)

CH-1196 Gland, Switzerland

Director General: Lee M. Talbot

G International Union of Geological Sciences (IUGS)

Maison de la Geologie

77 , rue Claude Bernard

75005 Paris, France

Secretary General: C. C. Weber

G,C International Union of Geodesy and Geophysics (IUGG)

Observatoire Royal de Belgique

Avenue Circulaire 3

1180 Bruxelles, Belgium

Secretary General: Paul Melchior

G,H International Union for Quaternary Research (INQUA)

Institute of Foundation Engineering

ETH-Honggerberg

CH-8039 Zurich, Switzerland

Secretary-Treasurer: Dr. Cristian Schluchter

H

International Water Resources Association (IWRA)

Science Complex Building

University of Wisconsin

Milwaukee, Wisconsin 53201 U.S.A.

H Inter-Union Commission on the Lithosphere (ICL)

NASA Headquarters, Code EL-4

Washington, D.C. 20546 U.S.A.

Secretary General: E. A. Flinn

H
Land and Water Development Division

Food and Agriculture Organization (FAO)

Viale delle Terme di Caracalla

00100 Rome, Italy
H

Natural Resources Division

Economic Commission for Africa (ECA)

P.O. Box 3001

Addis Ababa, Ethiopia

H Division of Natural Resources and Energy

Economic Commission for Latin America (ECLA)

Edificio Haciones Unidas

Casilla 179 D

Santiago, Chile

Officer in charge: Axel Dourojeanni

G,H Organization for Tropical Studies (OTS)

North American Office

P.O. Box DM, Duke Station

Durham, North Carolina 27706 U.S.A.

Executive Director: Donald E. Stone

G,C,H Pacific Science Association

P.O. Box 17801

Honolulu, Hawaii 96817 U.S.A.

Secretary General: Brenda Bishop

G,C,H Pan American Institute of Geography and History (PAIGH)

Ex-Arzobispado 29

Col. Observatorio

11860 Mexico 18 DF, Mexico

Secretary General: Jose A. Saenz G.

$\mathrm{H}$

$\mathbf{R}$

Resources and Transport Division

United Nations (UN)

New York, N.Y. 10017 U.S.A.

Saudi-Sudanese Red Sea Joint Commission

P.O. Box 5886

Jiddah-21432, Saudi Arabia

Deputy Secretary General: Dr. Zohair A. Nawab

H Scientific Affairs Division

North Atlantic Treaty Organization (NATO).

B-1110 Brussels, Belgium

Seismological Society of the South-West Pacific (SSSWP)

Seismological Observatory

P.O. Box 8005

Wellington, New Zealand

Governor: G. A. Eiby

G

Subcommission for the Metallogenic Map of the World

U.S. Geological Survey, MS 952

Reston, Virginia 22092 USA

President: Philip W. Guild 
U.S.S.R. Academy of Science

Palais des Nations

Pyzhevsky per. 7

Moscow 109017, U.S.S.R.

President: A.V. Peive

Secretary General: V. E. Khain

G,H,C United Nations Development Programme (UNDP)

Bureau for Programme, Policy and Evaluation

Room DC1-2072

One UN Plaza

New York, N.Y. 10017 U.S.A.

Principal Technical Advisor (Mineral Resources): Willem O. J. Groeneveld-Meijer

United Nations Environment Program (UNEP) Information Service

P. O. Box 30552

Nairobi, Kenya

G,C,H United Nations Educational, Scientific and Cultural Organization (UNESCO)

Division of Earth Sciences

7, place de Fontenoy

75700 Paris, France

Director: V. Sibrava

World Health Organization (WHO)

1211 Geneva 27, Switzerland

H

World Meteorological Organization (WMO)

P.O. Box No. 5

CH-1211 Geneva 20, Switzerland

Secretary General: G. O. P. Obasi 

